\title{
Search for pair production of second-generation leptoquarks at $\sqrt{s}=13 \mathrm{TeV}$
}

\author{
A. M. Sirunyan et al.* \\ (CMS Collaboration)
}

(Received 15 August 2018; published 27 February 2019)

\begin{abstract}
A search for pair production of second-generation leptoquarks is performed using proton-proton collision data collected at $\sqrt{s}=13 \mathrm{TeV}$ in 2016 with the CMS detector at the CERN LHC, corresponding to an integrated luminosity of $35.9 \mathrm{fb}^{-1}$. Final states with two muons and two jets, or with one muon, two jets, and missing transverse momentum are considered. Second-generation scalar leptoquarks with masses less than $1530(1285) \mathrm{GeV}$ are excluded for $\beta=1.0(0.5)$, where $\beta$ is the branching fraction for the decay of a leptoquark to a charged lepton and a quark. The results of the search are also interpreted as limits on the pair production of long-lived top squarks in an $R$-parity violating supersymmetry model that has a final state with two muons and two jets. These limits represent the most stringent limits to date on these models.
\end{abstract}

DOI: $10.1103 /$ PhysRevD.99.032014

\section{INTRODUCTION}

The standard model (SM) of particle physics displays a symmetry between the quark and lepton families. Leptoquarks (LQs) are new bosons that would manifest a fundamental connection between quarks and leptons and are predicted by numerous extensions of the SM, such as grand unified theories [1-8], composite models with lepton and quark substructure [9], technicolor models [10-12], and superstring-inspired models [13]. LQs are color-triplet scalar or vector bosons carrying both lepton and baryon numbers, and they decay either to a charged lepton and a quark or to a neutrino and a quark. Interpretations of direct searches for LQs are typically based on a general model where LQ-lepton-quark interactions are added to the Lagrangian [14]. Recently, interest in LQs has increased as they may provide an explanation for the observation of anomalies in the decays of $B$ mesons by the Belle [15-17], $B A B A R$ [18,19], and LHCb [20-23] collaborations.

At hadron colliders, LQs can be produced singly or in pairs. This analysis concentrates on pair production of scalar LQs. The dominant leading-order (LO) processes for pair production of LQs at the LHC involve gluon-gluon fusion and quark-antiquark annihilation, shown in Fig. 1.

The interactions of scalar LQs with SM particles are completely determined by three parameters [14]: the LQ mass $m_{\mathrm{LQ}}$, the Yukawa coupling at the LQ-lepton-quark vertex $\lambda_{\mathrm{LQ}}$, and the branching fraction $\beta$ of the LQ decay to

${ }^{*}$ Full author list given at the end of the article.

Published by the American Physical Society under the terms of the Creative Commons Attribution 4.0 International license. Further distribution of this work must maintain attribution to the author(s) and the published article's title, journal citation, and DOI. Funded by SCOAP . a charged lepton and a quark. The decay of a LQ to a neutrino and a quark is complementary to the decay to a charged lepton and a quark and has a branching fraction of $1-\beta$. Vector LQs are further dependent on two couplings which relate to the anomalous magnetic and electric quadrupole moments of the vector LQ [24].

As can be seen in Fig. 1, the dominant pair production processes have no LQ-lepton-quark vertices, and thus the production cross sections do not depend on $\lambda_{\mathrm{LQ}}$. The mean lifetime of the LQ is dependent on $\lambda_{\mathrm{LQ}}$. For $\lambda_{\mathrm{LQ}} \gtrsim 10^{-6.5}$ [14], TeV-scale LQs will have decay lengths that are less than the resolution of the impact parameter measurement of the CMS detector [25]. As is customary, the value of $\lambda_{\mathrm{LQ}}$ has been set such that $\lambda_{\mathrm{LQ}}^{2} /(4 \pi)=\alpha_{\mathrm{em}}$, where $\alpha_{\mathrm{em}}$ is the electromagnetic coupling. Therefore the LQs considered in this analysis always decay very close to the point of production and are referred to as prompt. As a consequence, the limits set on the pair production cross sections can be considered independent of $\lambda_{\mathrm{LQ}}$ for $\lambda_{\mathrm{LQ}} \gtrsim 10^{-6.5}$.

Pair production of LQs is characterized by final states with two leptons and two jets with large transverse momentum $p_{\mathrm{T}}$. This analysis assumes no flavor mixing between generations, to be consistent with experimental constraints on lepton flavor violation and flavor-changing neutral currents [26,27]. In this scenario, second-generation LQs will always decay to either a muon and a charm quark or to a neutrino and a strange quark. Values of 1.0 and 0.5 are considered for $\beta$, corresponding to maximal production of the two final states $\mu \mu \mathrm{jj}$ and $\mu \nu \mathrm{jj}$. Previous limits on second-generation scalar LQ pair production have been published by the CMS and ATLAS collaborations [28,29]. The CMS result excludes LQs with $m_{\mathrm{LQ}}<1080(760) \mathrm{GeV}$ for $\beta=1.0(0.5)$, in proton-proton $(p p)$ collisions at $8 \mathrm{TeV}$, and ATLAS excludes LQs with $m_{\mathrm{LQ}}<1160 \mathrm{GeV}$ for 

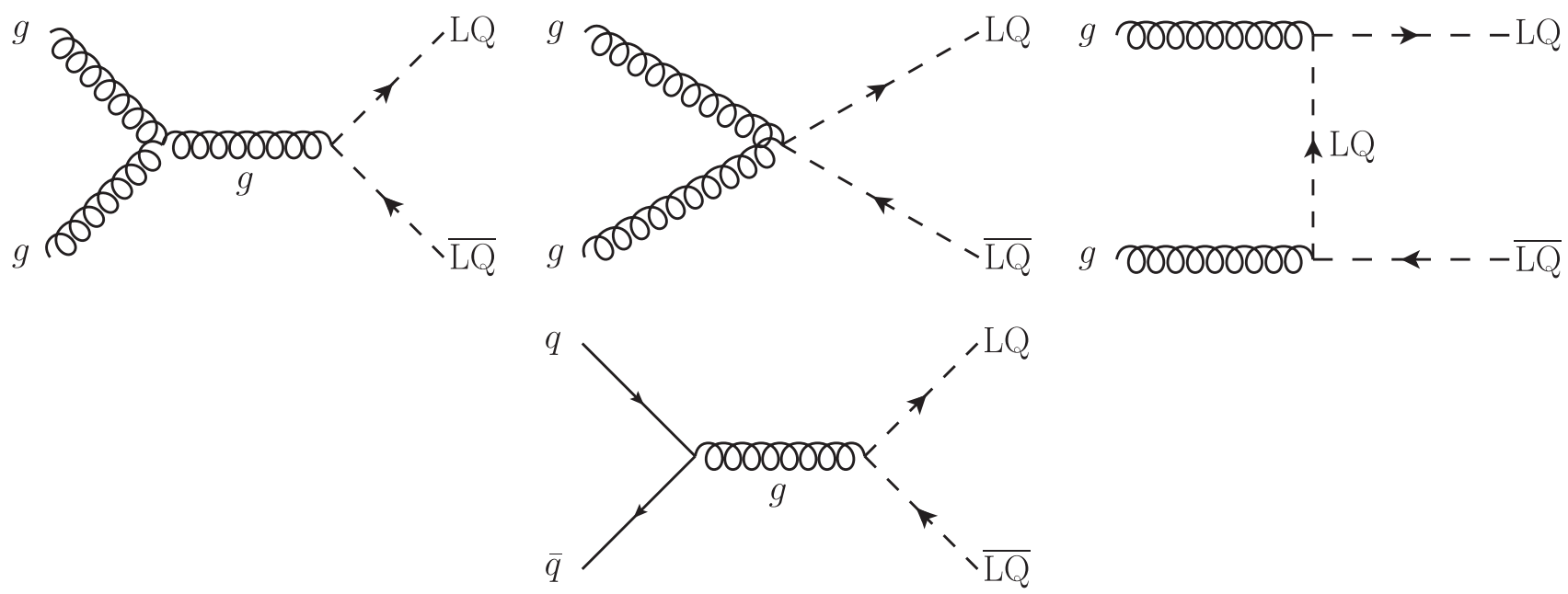

FIG. 1. Dominant leading-order Feynman diagrams for the pair production of LQs at the LHC.

$\beta=1.0$, at $13 \mathrm{TeV}$. The most stringent limits on vector LQs have been reported by CMS [28].

Other models of physics beyond the SM, such as $R$-parity violating (RPV) supersymmetry (SUSY) [30], can lead to the same final states as LQ production. Supersymmetry postulates a symmetry between fermions and bosons, which gives rise to superpartner particles for all known SM particles. In some SUSY scenarios, one of the two top quark superpartners (top squark, $\tilde{t}$ ) is the lightest SUSY particle and when $R$-parity is violated can decay to a bottom (b) quark and a charged lepton. For $\tilde{t}$ pair production and direct $\tilde{t}$ decays to a charged lepton $+b$ quark, limits can be extracted directly from the $L Q$ results. If the couplings of the RPV operators are sufficiently small, however, the superpartners will have long lifetimes and will travel through part or all of the detector before decaying. In this scenario, referred to in this paper as displaced SUSY [31], the $\tilde{t}$ has a finite but nonzero lifetime and decays to a charged lepton of any flavor and a bottom quark within a distance, $c \tau$, between 0.1 and $100 \mathrm{~cm}$, where $\tau$ is the $\tilde{t}$ mean lifetime. We assume the $\tilde{t}$ decays with equal probability to electrons, muons, and tau leptons. This analysis is sensitive to the low-lifetime, high-mass region of phase space where dedicated searches for displaced SUSY lose sensitivity [32].

\section{THE CMS DETECTOR}

The central feature of the CMS apparatus is a superconducting solenoid of $6 \mathrm{~m}$ internal diameter, providing a magnetic field of $3.8 \mathrm{~T}$. Within the solenoid volume are a silicon pixel and strip tracker, a lead tungstate crystal electromagnetic calorimeter (ECAL), and a brass and scintillator hadron calorimeter, each composed of a barrel and two end cap sections. Forward calorimeters extend the pseudorapidity coverage provided by the barrel and end cap detectors. Muons are measured in gas-ionization detectors embedded in the steel flux-return yoke outside the solenoid. A more detailed description of the CMS detector, together with a definition of the coordinate system used and the relevant kinematic variables, can be found in Ref. [33].

Events of interest are selected using a two-tiered trigger system [34]. The first level (L1), composed of custom hardware processors, uses information from the calorimeters and muon detectors to select events at a rate of around $100 \mathrm{kHz}$ within a time interval of less than $4 \mu \mathrm{s}$. The second level, known as the high-level trigger (HLT), consists of a farm of processors running a version of the full event reconstruction software optimized for fast processing and reduces the event rate to around $1 \mathrm{kHz}$ before data storage.

\section{DATA AND SIMULATED SAMPLES}

The dataset used in this paper was collected by CMS during the $2016 p p$ LHC run at $\sqrt{s}=13 \mathrm{TeV}$ and corresponds to an integrated luminosity of $35.9 \pm 0.9 \mathrm{fb}^{-1}$ [35]. Events are selected using triggers that require at least one muon with $p_{\mathrm{T}}>50 \mathrm{GeV}$, with no isolation requirements. These triggers supply the data for the $\mu \mu \mathrm{jj}$ and $\mu \nu \mathrm{jj}$ channels, as well as for the $e \mu$ sample used in the $t \bar{t}+$ jets background estimate for the $\mu \mu \mathrm{jj}$ channel.

Signal samples are produced in $50 \mathrm{GeV}$ steps for scalar $m_{\mathrm{LQ}}$ between 200 and $2000 \mathrm{GeV}$ using an effective theory based on Ref. [14] at LO with PYTHIA 8.212 [36]. These samples are used to study the acceptance of the signal. The production cross sections, calculated using next-to-leadingorder (NLO) QCD corrections [37] with the CTEQ6L1 [38] LO and CTEQ6.6 [39] NLO parton distribution function (PDF) sets, are used for comparison with data in the limit setting procedure. The search limits are independent of $\lambda_{\mathrm{LQ}}$ for sufficiently large values of $\lambda_{\mathrm{LQ}}$, as discussed in Sec. I. Displaced SUSY samples are produced with PYTHIA 8.212 using the Snowmass "Points and Slopes point 1a" parameter set [40] for $\tilde{t}$ masses from 200 to $1200 \mathrm{GeV}$, in $100 \mathrm{GeV}$ steps, and for $c \tau=0.1,1,10$, and $100 \mathrm{~cm}$. The lighter, left-handed top squark is the lightest 
supersymmetric particle (LSP) in this model, while the heavier right-handed top squark has a mass beyond the relevant kinematic regime. Production cross sections for $\tilde{t}$ are calculated at NLO+ next-to-leading logarithmic (NLL) precision with PROSPINO version 2 [41] and NLL-fast programs version $3.0[42,43]$, using the CTEQ6L1 PDF set.

Standard model backgrounds considered include $Z / \gamma^{*}+$ jets, $t \bar{t}+$ jets, $W+$ jets, single top quark production, and diboson $(W W / W Z / Z Z)+$ jets. The $Z / \gamma^{*}+$ jets, $W+$ jets, and diboson samples are generated at NLO using MADGRAPH5_amc@NLO version 2.3.3 [44,45]. Single top quark and $t \bar{t}+$ jets samples are generated at NLO using POWHEG v2 [46-49] and MADGRAPH5_aMC@NLO [50]. All backgrounds use PYTHIA 8.212 for fragmentation and hadronization.

The $W+$ jets and $Z / \gamma^{*}+$ jets samples are normalized to next-to-next-to-leading-order (NNLO) inclusive cross sections calculated with FEWZ versions 3.1 and 3.1.b2, respectively [51]. Single top quark and diboson samples are normalized to NLO inclusive cross sections calculated with MCFM version 6.6 [52-55]. The $t \bar{t}+$ jets sample is normalized to calculations at the NNLO level in QCD including resummation of next-to-next-to-leading logarithmic (NNLL) soft gluon terms produced with Top ++2.0 [56-62].

Signal and background events are generated using the NNPDF3.0 PDF sets [63], with the full CMS detector geometry and response simulated using GEANT4 [64,65]. All samples use the CUETP8M1 underlying event tune [66], with additional $p p$ interactions (the pileup distribution) overlaid and corrected to match the distribution measured in data.

The simulated samples are corrected so that the detector response and resolution for both leptons and jets and the triggering efficiency match those measured in data.

\section{EVENT RECONSTRUCTION AND SELECTION}

The CMS particle-flow event algorithm [67] aims to reconstruct and identify each individual particle in an event, with an optimized combination of information from the various elements of the detector. The reconstructed vertex with the largest value of summed physics-object $p_{\mathrm{T}}^{2}$ is taken to be the primary $p p$ interaction vertex. The physics objects in this context are jets clustered using the jet finding algorithm with all tracks assigned to the vertex as inputs, and the associated missing transverse momentum $\vec{p}_{\mathrm{T}}^{\text {miss }}$, taken as the negative vector sum of the $p_{\mathrm{T}}$ of those jets. The magnitude of the $\vec{p}_{\mathrm{T}}^{\text {miss }}$ is referred to as $p_{\mathrm{T}}^{\text {miss }}$.

Hadronic jets are reconstructed using the anti- $k_{\mathrm{T}}$ algorithm $[68,69]$ with a size parameter of 0.4 . Jet momentum is determined as the vectorial sum of all particle momenta in the jet, and it is found from simulation to be within 5\% to $10 \%$ of the true momentum over the whole $p_{\mathrm{T}}$ spectrum and detector acceptance. Additional $p p$ interactions within the same or nearby bunch crossings can contribute additional tracks and calorimetric energy depositions, increasing the apparent jet momentum. To mitigate this effect, tracks identified to be originating from pileup vertices are discarded, and an offset correction is applied to correct for remaining contributions. Jet energy corrections are derived from simulation to bring the measured response of jets to that of particle level jets on average. In situ measurements of the momentum balance in dijet, photon + jet, $Z+$ jet, and multijet events are used to determine any residual differences between the jet energy scale in data and in simulation and appropriate corrections are made [70]. These jet energy corrections are propagated to the $p_{\mathrm{T}}^{\text {miss }}$. Additional selection criteria are applied to each jet to remove jets potentially dominated by instrumental effects or reconstruction failures. Jets are required to have pseudorapidity $|\eta|<2.4, p_{\mathrm{T}}>50 \mathrm{GeV}$ and to be separated from all selected muons by $\Delta R>0.5$, where $\Delta R=$ $\sqrt{(\Delta \eta)^{2}+(\Delta \phi)^{2}}$ and $\phi$ is the azimuthal angle in radians. At least two jets are required for both the $\mu \mu \mathrm{jj}$ and $\mu \nu \mathrm{jj}$ channels, with no jet flavor requirement. Jets originating from $b$ quarks are used to estimate backgrounds in data control regions, and they are identified using the combined secondary vertex algorithm [71]. Jets are considered as $b$-tagged if they pass the "loose" working point, with an $80 \% b$ jet identification efficiency and a $10 \%$ rate of erroneous $b$ jet identification. Simulated samples are corrected on a jet-by-jet basis using correction factors to agree with $b$-tagged distributions measured in data.

Muons are measured in the pseudorapidity range $|\eta|<$ 2.4 in concentric stations with detection planes made using three technologies: drift tubes, cathode strip chambers, and resistive plate chambers. Hits in the muon tracking system are combined into hit segments. Muons are reconstructed as tracks by combining these hit segments with hits in the silicon tracker, with a reconstruction optimized for high $p_{\mathrm{T}}$ muons. Matching muons to tracks measured in the silicon tracker results in a relative $p_{\mathrm{T}}$ resolution for muons with $p_{\mathrm{T}}<100 \mathrm{GeV}$ of $1 \%$ in the barrel and $3 \%$ in the end caps. The $p_{\mathrm{T}}$ resolution in the barrel and end caps is better than $10 \%$ for muons with $p_{\mathrm{T}}$ up to $1 \mathrm{TeV}$ [72]. Muons are required to have $p_{\mathrm{T}}>53 \mathrm{GeV}$ and $|\eta|<2.4$ to be fully efficient with respect to the trigger, and they are required to satisfy a set of identification criteria optimized for high $p_{\mathrm{T}}$. Segments in at least two muon stations are required to be geometrically matched to a track in the silicon tracker, with at least one hit from a muon chamber in each station included in the muon track fit. In order to suppress muons from hadron decays and to allow for a more precise $p_{\mathrm{T}}$ measurement, at least five strip tracker layers with hits associated with the muon are required, and at least one hit in the pixel detector. To reject muons from cosmic rays, the transverse impact parameter of the muon track with respect to the primary vertex is required to be less than $2 \mathrm{~mm}$, and the longitudinal distance of the track with respect to the 
primary vertex is required to be less than $5 \mathrm{~mm}$. An isolation requirement is imposed, as the signal produces isolated muons. The $p_{\mathrm{T}}$ sum of all tracks from the primary vertex (excluding the muon track itself) in a cone of $\Delta R=$ 0.3 around the muon track, divided by the muon $p_{\mathrm{T}}$, is required to be less than 0.1 . This relative isolation is shown to be independent of pileup [72]. In the $\mu \mu \mathrm{jj}$ channel at least two muons are required, with no charge requirement. In the $\mu \nu \mathrm{jj}$ channel exactly one muon is required.

Electrons are measured in the pseudorapidity range $|\eta|<2.5$. The electron momentum is estimated by combining the energy measurement in the ECAL with the momentum measurement in the tracker. The momentum resolution for electrons with $p_{\mathrm{T}} \approx 45 \mathrm{GeV}$ from $Z \rightarrow e e$ decays ranges from $1.7 \%$ to $4.5 \%$ [73]. In this analysis electrons are used as a control data sample for a $t \bar{t}+$ jets background estimate in the $\mu \mu \mathrm{jj}$ channel, and electrons with $p_{\mathrm{T}}>53 \mathrm{GeV}$ are vetoed in the $\mu \nu \mathrm{jj}$ channel to avoid overlap with this control region. With this high veto threshold the selection is kept as inclusive as possible for signal. The $t \bar{t}+$ jets background is small in the mass region of interest, which is above $1 \mathrm{TeV}$.

The LQ candidates are reconstructed using the pairing where the two reconstructed masses are closest. In the $\mu \mu \mathrm{jj}$ channel the two highest $p_{\mathrm{T}}$ muons and two highest $p_{\mathrm{T}}$ jets that pass the selection criteria above are considered. Each muon is paired with a jet in the configuration that minimizes the LQ- $\overline{\mathrm{LQ}}$ invariant mass difference. In the $\mu \nu \mathrm{jj}$ channel the two highest $p_{\mathrm{T}}$ jets are considered together with the required single muon. The muon and $\vec{p}_{\mathrm{T}}^{\text {miss }}$ are each paired with a jet in a similar manner to the $\mu \mu \mathrm{jj}$ channel, using instead the LQ transverse masses $m_{\mathrm{T}}^{\mathrm{LQ}}=\sqrt{2 p_{\mathrm{T}}^{\ell} p_{\mathrm{T}}^{\mathrm{jet}}(1-\cos [\Delta \phi(\ell, \mathrm{jet})])}$ of the muon-jet and $\vec{p}_{\mathrm{T}}^{\text {miss }}$-jet systems, where in this case $\ell$ represents the muon or neutrino in the decay. This method correctly matches the decay products of the two LQs in 50\% to $70 \%$ of signal events, increasing with $m_{\mathrm{LQ}}$.

\section{ESTIMATION OF STANDARD MODEL BACKGROUNDS}

\section{A. The $\mu \mu \mathrm{j} \mathbf{j}$ channel}

The main backgrounds that can mimic the LQ signal in the $\mu \mu \mathrm{jj}$ channel are $Z / \gamma^{*}+$ jets and $t \bar{t}+$ jets events.

Backgrounds are estimated and validated using a selection dominated by background events, referred to as the preselection. The preselection applies criteria that are looser than any final selection. This preselection requires at least two muons with $p_{\mathrm{T}}>53 \mathrm{GeV}$ and at least two jets with $p_{\mathrm{T}}>50 \mathrm{GeV}$. The muons are required to be separated from one another by $\Delta R>0.3$. The invariant mass of the dimuon system $\left(m_{\mu \mu}\right)$ is required to be greater than $50 \mathrm{GeV}$, and the $S_{\mathrm{T}}^{\mu \mu \mathrm{jj}}$ of the event is required to be greater than
$300 \mathrm{GeV}$, where $S_{\mathrm{T}}^{\mu \mu \mathrm{jj}}$ is defined as the scalar sum of the $p_{\mathrm{T}}$ of the two jets and two muons in the event.

The $Z / \gamma^{*}+$ jets background is estimated with events that satisfy the preselection, in a data control region around the $Z$ peak that is not in the search region. The background shape is taken from simulation, which shows good shape agreement with the data in the control region. For normalization, the simulation is compared to data in a window $80<M_{\mu \mu}<100 \mathrm{GeV}$ around the $Z$ peak, and a measured data normalization scale factor of $0.98 \pm 0.01$ (stat) \pm 0.09 (syst) is applied to simulated events passing the final selection criteria. The systematic uncertainty is assigned to account for the dependence of the scale factor on event kinematic properties. All final selections require $M_{\mu \mu}>100 \mathrm{GeV}$, to reduce the $Z / \gamma^{*}$ background, and to maintain the separation of the control region from the search region.

The $t \bar{t}+$ jets background is estimated using an independent $e \mu$ data sample. Events are selected that contain one electron and one muon, and must satisfy all requirements of the $\mu \mu \mathrm{jj}$ preselection, other than the normal two muon requirement. No charge requirement is placed on the electron and muon. This sample is corrected for differences between the $\mu \mu$ and $e \mu$ selection, such as those based on identification and isolation, as well as on trigger efficiency. The kinematic distributions of this sample are found to be in good agreement with the $t \bar{t}+$ jets simulation, and use of the $e \mu$ control sample in data reduces the systematic uncertainties associated with this background.

Background contributions from single top quark, $W+$ jets, and diboson events are estimated from simulation. Background from QCD multijets is shown to be negligible using data control regions.

Background predictions are validated at the preselection level by comparing them with data. Good agreement is seen in all relevant kinematic distributions. Three kinematic variables are identified that have strong discrimination power between signal and background. In the $\mu \mu \mathrm{jj}$ channel, these variables are $S_{\mathrm{T}}^{\mu \mu \mathrm{jj}}, m_{\mu \mu}$, and $m_{\mu \mathrm{j}}^{\min }$, where $m_{\mu \mathrm{j}}^{\min }$ is defined as the smaller of the two muon-jet invariant masses that represent the LQ and $\overline{\mathrm{LQ}}$ candidates. A comparison of these main kinematic variables is shown in Fig. 2 at the preselection level.

\section{B. The $\mu \nu \mathbf{j j}$ channel}

As in the $\mu \mu \mathrm{jj}$ channel, a background-dominated preselection is used to calculate and validate the SM background estimates. This preselection requires exactly one muon with $p_{\mathrm{T}}>53 \mathrm{GeV}$ and at least two jets with $p_{\mathrm{T}}>50 \mathrm{GeV}$. The direction of the muon in the event is required to be separated from $\vec{p}_{\mathrm{T}}^{\text {miss }}$ by $\Delta \phi>0.8$, and the momentum vector of the highest- $p_{\mathrm{T}}$ jet to be separated from $\vec{p}_{\mathrm{T}}^{\text {miss }}$ by $\Delta \phi>0.5$. Further requirements include $m_{\mathrm{T}}^{\mu \nu}>50 \mathrm{GeV}, p_{\mathrm{T}}^{\text {miss }}>55 \mathrm{GeV}$, and $S_{\mathrm{T}}^{\mu \nu \mathrm{jj}}>300 \mathrm{GeV}$, 

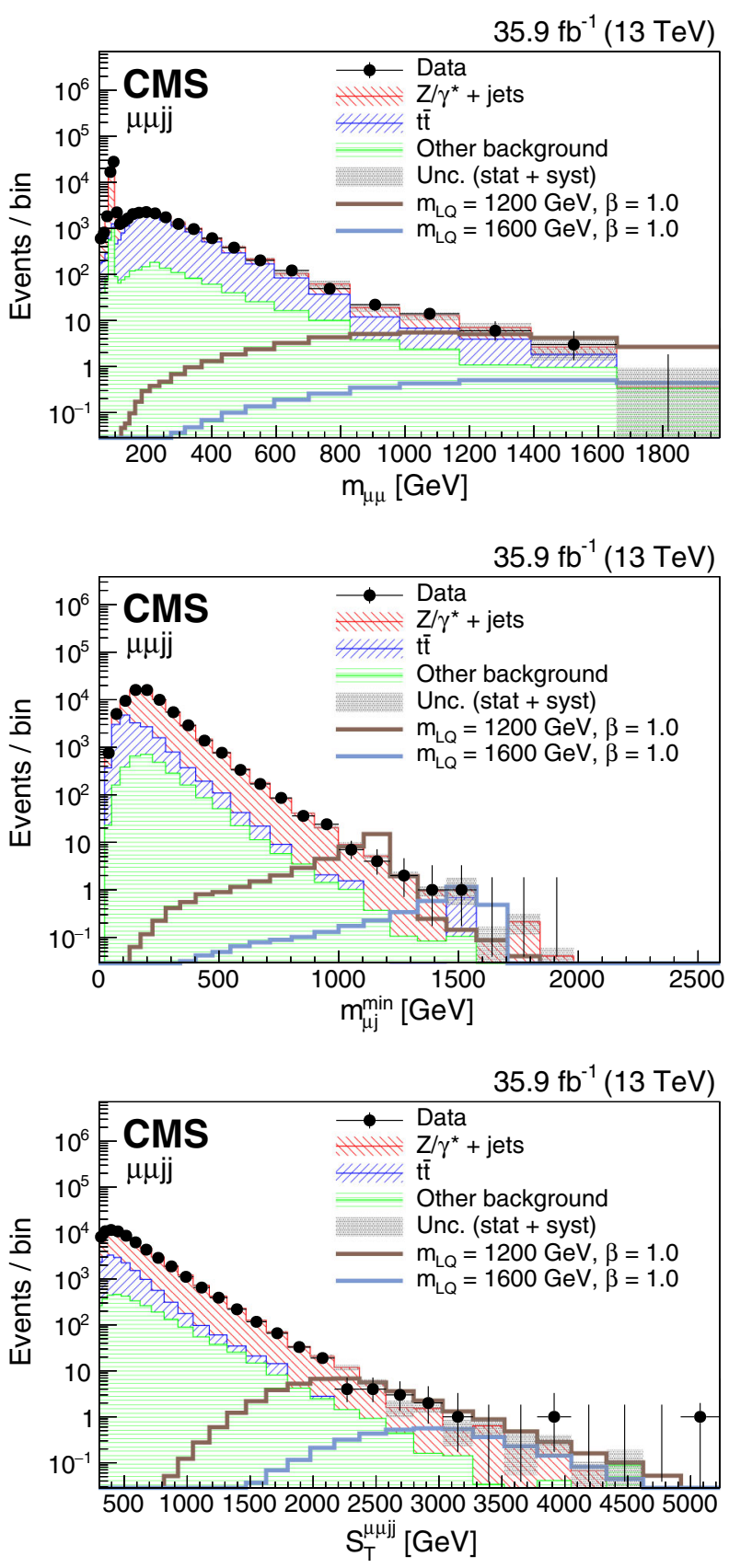

FIG. 2. Comparison of data and background at the preselection level for the $\mu \mu \mathrm{jj}$ channel, for the variables used for the final selection optimization: $m_{\mu \mu}$ (upper), $m_{\mu \mathrm{j}}^{\min }$ (middle), and $S_{\mathrm{T}}^{\mu \mu \mathrm{jj}}$ (lower). "Other background" includes $W+$ jets, single top quark, and diboson backgrounds. The hashed band represents the combined statistical and systematic uncertainty in the full background estimate.

where $S_{\mathrm{T}}^{\mu \nu \mathrm{jj}}$ is defined as the scalar sum of the $p_{\mathrm{T}}$ of the two jets matched to leptons $(\ell=\mu, \nu)$, the muon, and the $p_{\mathrm{T}}^{\text {miss }}$ in the event.

The main backgrounds that can mimic the LQ signal in the $\mu \nu \mathrm{jj}$ channel are $W+$ jets and $t \bar{t}+$ jets events. Both backgrounds are calculated using simulated samples normalized to the number of events in two separated data control regions. They are estimated with events that, in addition to satisfying the $\mu \nu \mathrm{jj}$ preselection, also satisfy $70<M_{\mathrm{T}}^{\mu \nu}<110 \mathrm{GeV}$. The events are then separated into two control regions, further enriched in their respective background processes, using $b$ tagging. The $W+$ jets background control region requires no $b$-tagged jets, while the $t \bar{t}+$ jets control sample requires at least one $b$-tagged jet. The $W+$ jets data normalization scale factor is found to be $0.93 \pm 0.01$ (stat), and the $t \bar{t}+$ jets data normalization scale factor is found to be $0.98 \pm 0.01$ (stat). As the scale factors do not depend on the kinematic distributions, no further systematic uncertainty is applied. These data normalization scale factors are then applied to simulated events passing the final selections.

Backgrounds from single top quark, $Z / \gamma^{*}+$ jets, and diboson events are estimated from simulation. Background from QCD multijets is shown to be negligible using data control regions.

After preselection, discriminating variables are identified, as with the $\mu \mu \mathrm{jj}$ channel. In the $\mu \nu \mathrm{jj}$ channel, these variables are $S_{\mathrm{T}}^{\mu \nu \mathrm{jj}}, m_{\mathrm{T}}^{\mu \nu}$, and $m_{\mu \mathrm{j}}$, where $m_{\mathrm{T}}^{\mu \nu}$ and $m_{\mu \mathrm{j}}$ are defined as the muon- $\vec{p}_{\mathrm{T}}^{\text {miss }}$ transverse mass and the muon-jet invariant mass for the combination that minimizes the LQ- $\overline{L Q}$ transverse mass difference. Distributions for these variables in events satisfying the preselection are shown in Fig. 3.

\section{FINAL SELECTION}

\section{A. Final selection optimization}

For both the $\mu \mu \mathrm{jj}$ and $\mu \nu \mathrm{jj}$ channels, the previously described kinematic variables identified as having strong discrimination power between signal and background are used to define a final selection for each $m_{\mathrm{LQ}}$. The signal-tobackground separation is optimized with a full threedimensional optimization using the Punzi significance [74] for a discovery potential of 5 standard deviations at 95\% confidence level (C.L.). This method is optimal for both making a discovery and for setting limits, and it is valid in cases with low background event counts. In the $\mu \mu \mathrm{jj}$ channel, the $m_{\mu \mu}$ is required to be greater than $100 \mathrm{GeV}$ to exclude the background control region. In the $\mu \nu \mathrm{jj}$ channel, the $m_{\mathrm{T}}^{\mu \nu}$ is required to be greater than $110 \mathrm{GeV}$ for the same reason. The lower bounds of the final selection criteria for the three variables are shown as a function of scalar $m_{\mathrm{LQ}}$ in Fig. 4. The behavior of the different variable responses to the optimization can be attributed to the shapes of the signal distributions of the different variables, as seen in Figs. 2 and 3.

\section{B. Systematic uncertainties}

Systematic uncertainties in the LQ signal production cross sections vary from $14 \%$ to $50 \%$ across the full LQ 

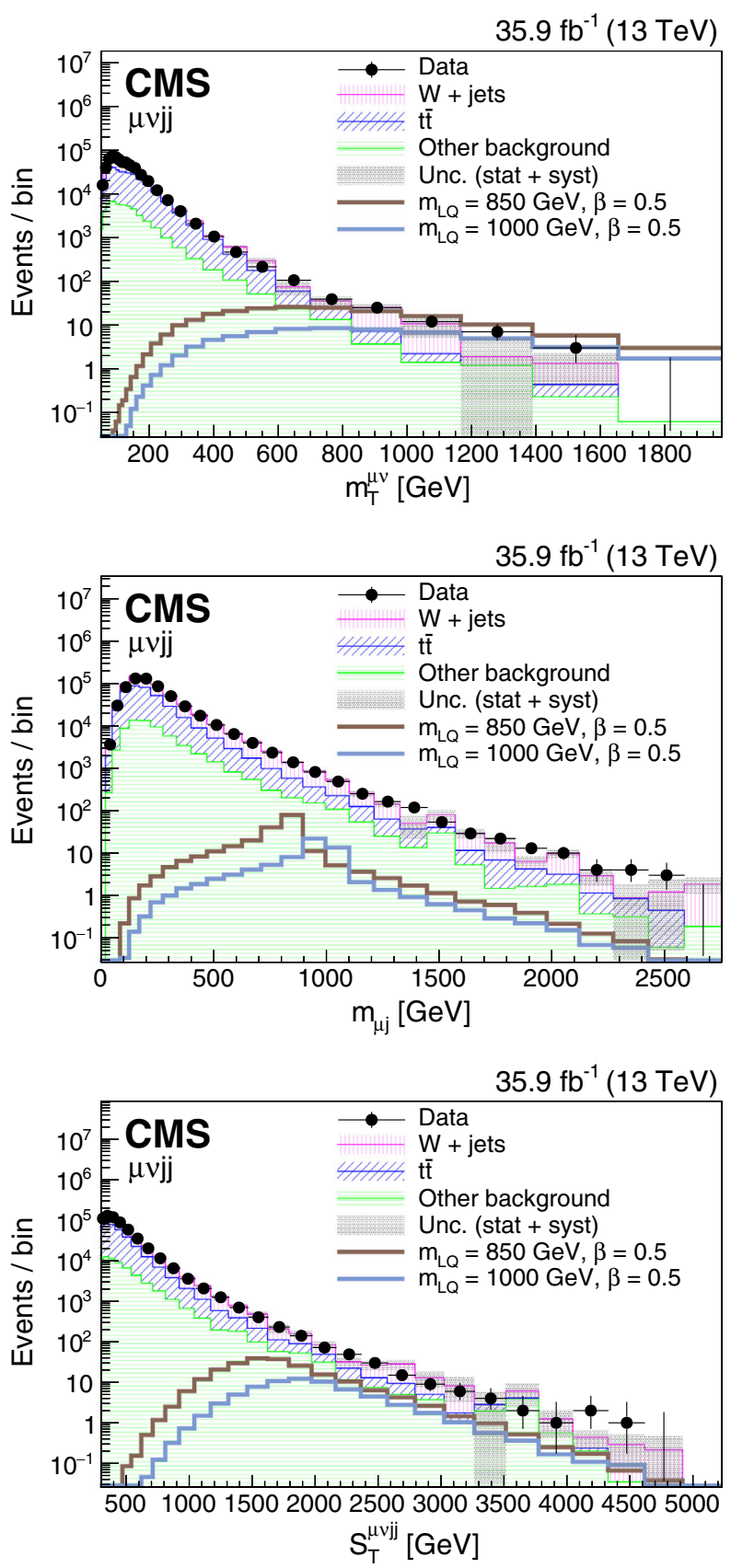

FIG. 3. Comparison of data and background at the preselection level for the $\mu \nu \mathrm{jj}$ channel, for the variables used for final selection criteria optimization: $m_{\mathrm{T}}^{\mu \nu}$ (upper), $m_{\mu \mathrm{j}}$ (middle), and $S_{\mathrm{T}}^{\mu \nu \mathrm{jj}}$ (lower). "Other background" includes $Z / \gamma^{*}+$ jets, single top quark, and diboson backgrounds. The hashed band represents the combined statistical and systematic uncertainty in the full background estimate.

mass range. They are estimated by varying the PDF eigenvectors within their uncertainties and the renormalization and factorization scales by factors of one-half and two.

Systematic uncertainties in the background yields and in the signal acceptance for both the $\mu \mu \mathrm{jj}$ and $\mu \nu \mathrm{jj}$
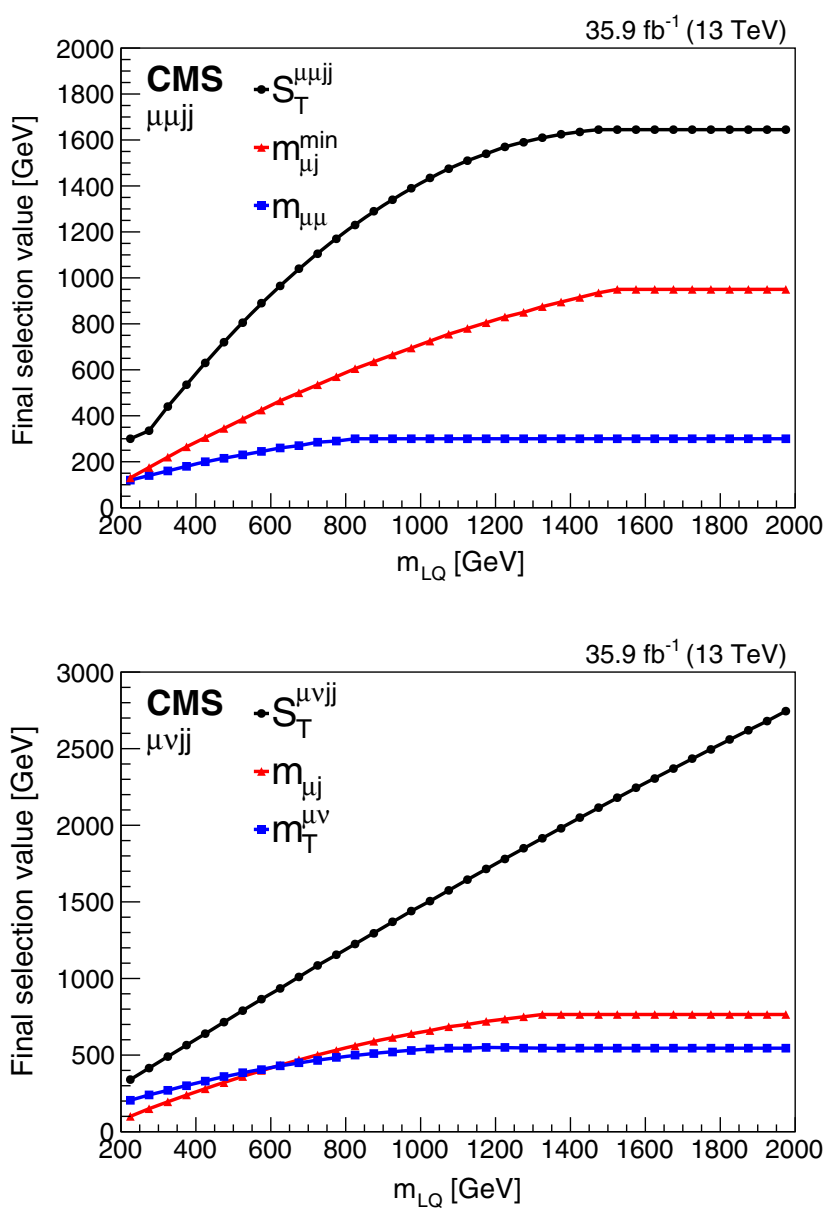

FIG. 4. Lower bounds of the final selection criteria for the three variables for the $\mu \mu \mathrm{jj}$ (upper) and $\mu \nu \mathrm{jj}$ (lower) channels as a function of scalar $m_{\mathrm{LQ}}$.

channels are calculated for each final selection by running the full analysis with separately varied detector quantities, particle momenta, or scale factors. These yields are compared to those for the nominal analysis, and the differences are propagated as log-normal nuisance parameters in the limit setting. The effects of these systematic uncertainties in signal acceptance and total background yield are shown for the $\mu \mu \mathrm{jj}$ and $\mu \nu \mathrm{jj}$ channels in Tables I and II, respectively.

Systematic uncertainties in the jet energy resolution and muon energy resolution are measured by smearing the jet and muon momenta, including high- $p_{\mathrm{T}}$ specific corrections for muons [75]. Uncertainties due to the jet energy scale and the muon energy scale are estimated by propagating jet and muon energy corrections.

Uncertainties in the shapes of the main backgrounds are estimated by varying the factorization and normalization scales in the simulation by factors of $1 / 2$ and 2 . These uncertainties, which include the uncertainty in the extrapolation of the distributions of the final selection variables from the control to signal regions, are estimated for the $Z / \gamma^{*}+$ jets, $t \bar{t}+$ jets, $W+$ jets, and diboson backgrounds. 
TABLE I. Range of systematic uncertainties in the signal acceptance and background yields for the $\mu \mu \mathrm{jj}$ analysis. The last two lines show the total systematic uncertainty and the total statistical uncertainty in the simulated samples, respectively.

\begin{tabular}{lcc}
\hline \hline$\mu \mu \mathrm{jj}$ uncertainty & Signal $(\%)$ & Background $(\%)$ \\
\hline Jet energy resolution & $0.0-0.4$ & $0.3-4.8$ \\
Jet energy scale & $0.1-1.8$ & $0.4-4.9$ \\
Integrated luminosity & $2.5-2.5$ & $0.3-0.9$ \\
Muon energy resolution & $0.0-0.2$ & $0.0-3.8$ \\
Muon energy scale & $0.0-0.2$ & $1.3-6.2$ \\
Muon ID/isolation & $6.1-6.8$ & $1.2-2.9$ \\
PDF & $1.9-4.0$ & $0.4-4.6$ \\
Pileup & $0.0-0.3$ & $0.2-5.9$ \\
Trigger & $0.1-0.7$ & $0.0-0.5$ \\
Tracking efficiency & $1.0-2.0$ & $0.1-0.9$ \\
$t \bar{t}+$ jets normalization & $\ldots$ & $0.0-0.3$ \\
$t \bar{t}+$ jets shape & $\ldots$ & $0.0-0.0$ \\
$W+$ jets normalization & $\ldots$ & $0.0-0.1$ \\
$W+$ jets shape & $\ldots$ & $0.0-0.0$ \\
$Z / \gamma^{*}+$ jets normalization & $\ldots$ & $3.4-7.3$ \\
$Z / \gamma^{*}+$ jets shape & $\ldots$ & $1.5-6.2$ \\
Diboson shape & $\ldots$ & $0.7-9.2$ \\
Total syst. uncertainty & $7.2-8.5$ & $5.0-12$ \\
Total stat. uncertainty & $0.5-1.0$ & $0.6-29$ \\
\hline \hline
\end{tabular}

In the $\mu \mu \mathrm{jj}$ channel the uncertainty in the $Z / \gamma^{*}+$ jets background normalization is estimated by varying the normalization scale factor described in Sec. VA up and down by its statistical and systematic uncertainties added

TABLE II. Range of systematic uncertainties in the signal acceptance and background yields for the $\mu \nu \mathrm{jj}$ analysis. The last two lines show the total systematic uncertainty and the total statistical uncertainty in the simulated samples, respectively.

\begin{tabular}{lcc}
\hline \hline$\mu \nu$ jj uncertainty & Signal $(\%)$ & Background $(\%)$ \\
\hline Jet energy resolution & $1.2-2.3$ & $3.4-6.1$ \\
Jet energy scale & $0.0-0.8$ & $0.7-6.7$ \\
Integrated luminosity & $2.5-2.5$ & $0.5-1.4$ \\
Muon energy resolution & $0.0-0.1$ & $0.2-4.7$ \\
Muon energy scale & $0.0-0.2$ & $0.4-2.9$ \\
Muon ID/isolation & $3.0-3.1$ & $0.5-2.5$ \\
PDF & $0.4-0.8$ & $0.9-5.6$ \\
Pileup & $0.0-0.3$ & $0.6-3.1$ \\
Trigger & $4.2-7.5$ & $0.8-5.5$ \\
Tracking efficiency & $0.5-1.0$ & $0.1-0.7$ \\
$b$ tagging efficiency & $\ldots$ & $1.4-3.6$ \\
$t \bar{t}+$ jets normalization & $\ldots$ & $0.1-0.5$ \\
$t \bar{t}+$ jets shape & $\ldots$ & $0.0-0.0$ \\
$W+$ jets normalization & $\ldots$ & $0.3-0.5$ \\
$W+$ jets shape & $\ldots$ & $1.6-8.7$ \\
$Z / \gamma^{*}+$ jets normalization & $\ldots$ & $0.6-1.4$ \\
$Z / \gamma^{*}+$ jets shape & $\ldots$ & $0.0-0.0$ \\
Diboson shape & $\ldots$ & $0.5-8.4$ \\
Total syst. uncertainty & $6.1-8.7$ & $6.6-13$ \\
Total stat. uncertainty & $0.1-1.3$ & $0.2-19$ \\
\hline \hline
\end{tabular}

in quadrature. The uncertainty in the $t \bar{t}+$ jets normalization is estimated by varying the $\mu \mu / e \mu$ correction factor up and down by its statistical uncertainty. In the $\mu \nu \mathrm{jj}$ channel the uncertainties in the $W+$ jets and $t \bar{t}+$ jets normalizations are estimated by varying the normalization scale factors described in Sec. VB up and down by their statistical uncertainties.

Other sources of systematic uncertainty considered are the luminosity measurement [35], muon identification and isolation [72], pileup [76], trigger efficiency, and track reconstruction efficiency. The uncertainty from the PDF prediction is estimated by varying the NNPDF3.0 eigenvectors within their uncertainties, following the PDF4LHC prescription $[77,78]$. A further uncertainty in the $b$ tagging efficiency is applied only in the $\mu \nu \mathrm{jj}$ channel [71], where the control region is defined via $b$ tagging. For most values of $m_{\mathrm{LQ}}$ the systematic uncertainties are at the lower end of the range. The maximum values given in Tables I and II are only relevant for large values of $m_{\mathrm{LQ}}$, where the total uncertainty is dominated by the statistical uncertainty in the simulated background samples.

\section{RESULTS}

\section{A. Data comparison with background after final selection}

The data are compared to background predictions after the final selections have been applied. Comparisons of the kinematic distributions, after the final selection, for data and simulation for two $m_{\mathrm{LQ}}$ hypotheses are shown in Fig. 5. No significant excess above the predicted background is seen for any $m_{\mathrm{LQ}}$, within uncertainties. The largest difference between data and the background estimate is a roughly two standard deviation excess in the $\mu \nu \mathrm{jj}$ channel for $m_{\mathrm{LQ}}=950 \mathrm{GeV}$. Kinematic distributions of the small excess of events in this region do not look like signal events, lacking the characteristic mass peak expected of LQs. There is one high- $S_{\mathrm{T}}^{\mu \mu \mathrm{jj}}$ event that can be seen in Fig. 5 (upper left) that merits mention. The background estimate for high-mass final selections for $S_{\mathrm{T}}^{\mu \mu \mathrm{jj}}>3000 \mathrm{GeV}$ is $0.0_{-0.0}^{+0.1}$. However, this event is unlike a signal event. In particular, the invariant masses of the two LQ candidates in this event are not compatible with LQ pair production.

Comparisons of background, data, and signal for each set of final selections can be seen in Figs. 6 and 7. The $y$ axis shows the final selection event yields for each of the individual $m_{\mathrm{LQ}}$ hypotheses shown on the $x$ axis. All the bins are correlated in these plots, as the events selected for each $m_{\mathrm{LQ}}$ are a strict subset of the events selected for the lower mass LQ. The product of acceptance and efficiency of the signal for all final selections, as well as detailed tables of the event counts in data, background, and signal, are shown in the Appendix. 

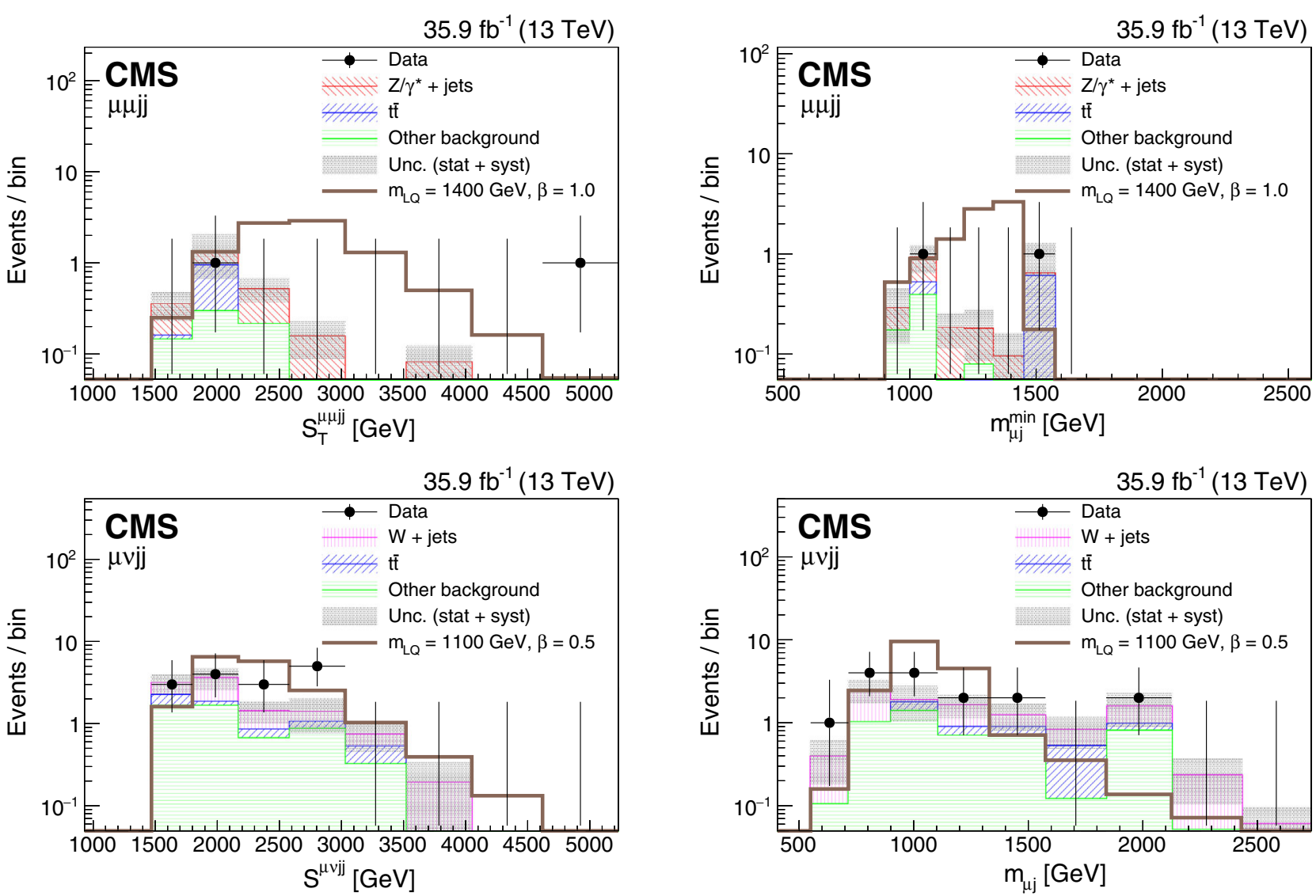

FIG. 5. Comparison of data and background distributions of $S_{\mathrm{T}}^{\mu \mu \mathrm{jj}}$ (left), $m_{\mu \mathrm{j}}^{\min }$ (upper right), and $m_{\mu \mathrm{j}}$ (lower right), for the $\mu \mu \mathrm{jj}$ channel (upper plots) and the $\mu \nu \mathrm{jj}$ channel (lower plots). Events after final selections with $m_{\mathrm{LQ}}=1400 \mathrm{GeV}$ are shown in the upper plots, and with $m_{\mathrm{LQ}}=1100 \mathrm{GeV}$ in the lower plots. The hashed band represents the combined statistical and systematic uncertainty in the full background estimate. "Other background" includes $W+$ jets, single top quark, and diboson backgrounds in the $\mu \mu \mathrm{jj}$ channel, and $Z / \gamma^{*}+$ jets, single top quark, and diboson backgrounds in the $\mu \nu \mathrm{jj}$ channel.

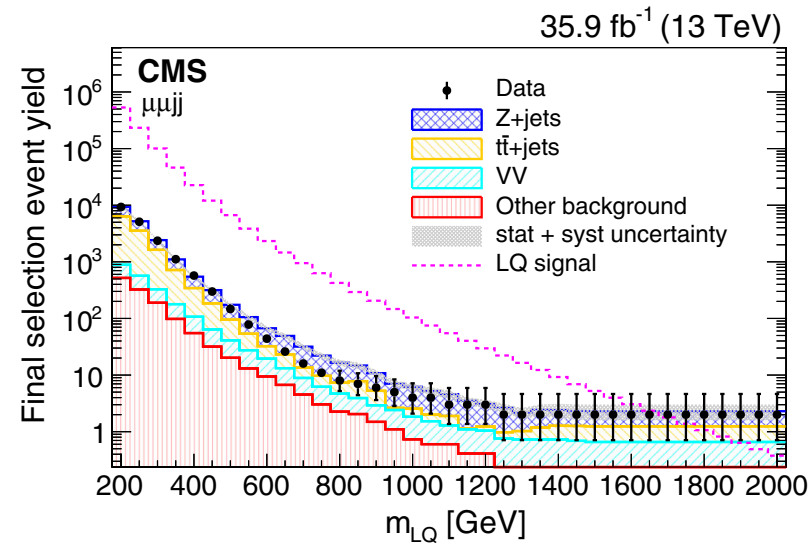

FIG. 6. Data and background event yields after final selections for the $\mu \mu \mathrm{jj}$ analysis, as a function of scalar $m_{\mathrm{LQ}}$. "Other background" includes $W+$ jets and single top quark production. The selection criteria for each bin are detailed in Table I. All the bins are correlated, as the events selected for each $m_{\mathrm{LO}}$ are a strict subset of the events selected for the lower mass LQ. The hashed band represents the combined statistical and systematic uncertainty in the full background estimate.

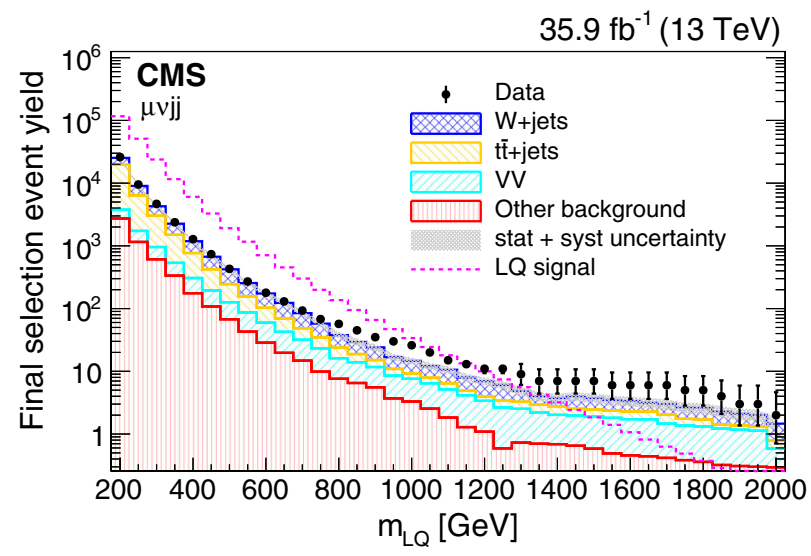

FIG. 7. Data and background event yields after final selections for the $\mu \nu \mathrm{jj}$ analysis, as a function of $m_{\mathrm{LQ}}$. "Other background" includes $Z / \gamma^{*}+$ jets and single top quark production. The selection criteria for each bin are detailed in Table II. All the bins are correlated, as the events selected for each $m_{\mathrm{LO}}$ are a strict subset of the events selected for the lower mass LQ. The hashed band represents the combined statistical and systematic uncertainty in the full background estimate. 


\section{B. Limit setting}

Limits are set on the LQ pair production cross section $\sigma$ as a function of scalar $m_{\mathrm{LQ}}$, using the asymptotic approximation [79] of the modified frequentist $\mathrm{CL}_{\mathrm{s}}$ approach [80,81], utilizing the ratio of the confidences in the signal+background to background hypotheses. The systematic uncertainties listed above are introduced as nuisance parameters in the limit setting procedure using log-normal probability functions. Uncertainties of a statistical nature are described by $\Gamma$ distributions with widths determined by the number of events in simulated samples or observed in data control regions. These limits have been compared to the so-called "LHC-style" fully frequentist $\mathrm{CL}_{\mathrm{s}}$ limits [82] and are found to be in good agreement with the expected and observed limits for all final selections, but with slightly more conservative systematic uncertainties in the low background regime.

The $95 \%$ C.L. upper limits on $\sigma \beta^{2}$ or $\sigma 2 \beta(1-\beta)$ as a function of scalar $m_{\mathrm{LQ}}$ are shown, together with the NLO predictions for the scalar LQ pair production cross section, in Fig. 8. Systematic uncertainties in the LQ signal production cross sections are shown as a band around the signal production cross section. By comparing the observed upper limit with the theoretical cross section values, second-generation scalar LQs with masses less than 1530 (1150) $\mathrm{GeV}$ are excluded under the assumption that $\beta=1.0(0.5)$, compared to the median expected limits of 1515(1260) GeV.

Limits are also set at $95 \%$ C.L. for $\beta$ values from 0 to 1 for both the $\mu \mu \mathrm{jj}$ and $\mu \nu \mathrm{jj}$ channels, as well as for the combination of both channels. In the combination, all systematic uncertainties are treated as fully correlated and all statistical uncertainties are treated as fully uncorrelated. The resulting two-dimensional limit plot is shown in Fig. 9. The combination of the two channels improves the mass exclusion, particularly for low values of $\beta$. Using the combined channels, second-generation scalar LQs with masses less than $1285 \mathrm{GeV}$ can be excluded for $\beta=0.5$, compared with an expected limit of $1365 \mathrm{GeV}$.

The results in the $\mu \mu \mathrm{jj}$ channel are also interpreted in the context of the displaced SUSY model described in Sec. I. Studies in both simulation and data have shown that tracking efficiency remains at $\sim 100 \%$ for the lifetimes and corresponding impact parameters considered [32], which allows interpretation of the results for a displaced signal to be made with the same final selections and systematic uncertainties as previously used for a prompt signal. The $95 \%$ C.L. expected and observed limits on the displaced SUSY $\tilde{t}$ pair production cross section are shown in Fig. 10. The limits are presented in two dimensions as a function of $\tilde{t}$ mass and lifetime. The expected and observed limits have been extrapolated to $c \tau=0 \mathrm{~cm}$ using the prompt LQ limits, taking into account the assumed $\tilde{t}$ branching ratio, $\tilde{t} \rightarrow \mathrm{b} \mu=1 / 3$. This extrapolation connects these results to the prompt kinematic range and is motivated by the fact that prompt top squark pair production is
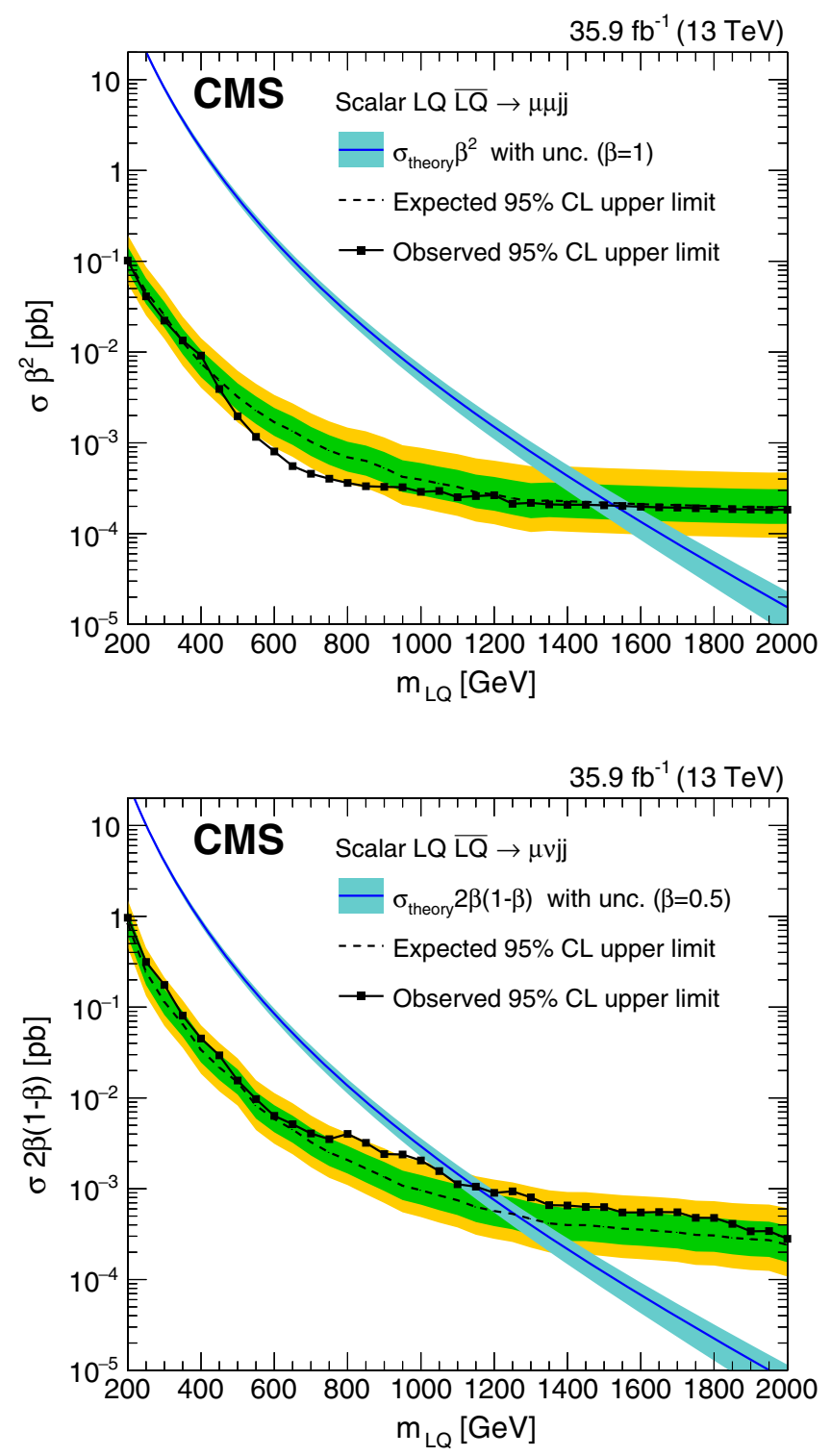

FIG. 8. The expected and observed upper limits at 95\% C.L. on the product of the scalar LQ pair production cross section and the branching fractions $\beta^{2}$ or $2 \beta(1-\beta)$ as a function of the second-generation $m_{\mathrm{LQ}}$ obtained with the $\mu \mu \mathrm{jj}$ (upper) or $\mu \nu \mathrm{jj}$ (lower) analysis. The solid lines represent the observed limits, the dashed lines represent the median expected limits, and the inner dark green and outer light yellow bands represent the $68 \%$ and $95 \%$ confidence intervals. The $\sigma_{\text {theory }}$ curves and their blue bands represent the theoretical scalar LQ pair production cross sections and the uncertainties on the cross sections due to the PDF prediction and renormalization and factorization scales, respectively.

kinematically very similar to that for LQs. The observed exclusion limits are 1150,940 , and $305 \mathrm{GeV}$ for $c \tau=0.1$, 1.0 , and $10.0 \mathrm{~cm}$. Following the formulation in Ref. [83] these limits can be translated into lower bounds on the coupling strength of the RPV term in the SUSY Lagrangian, in this case $\lambda_{233}^{\prime} \cos (\theta)$, where $\cos (\theta)$ represents the mixing 


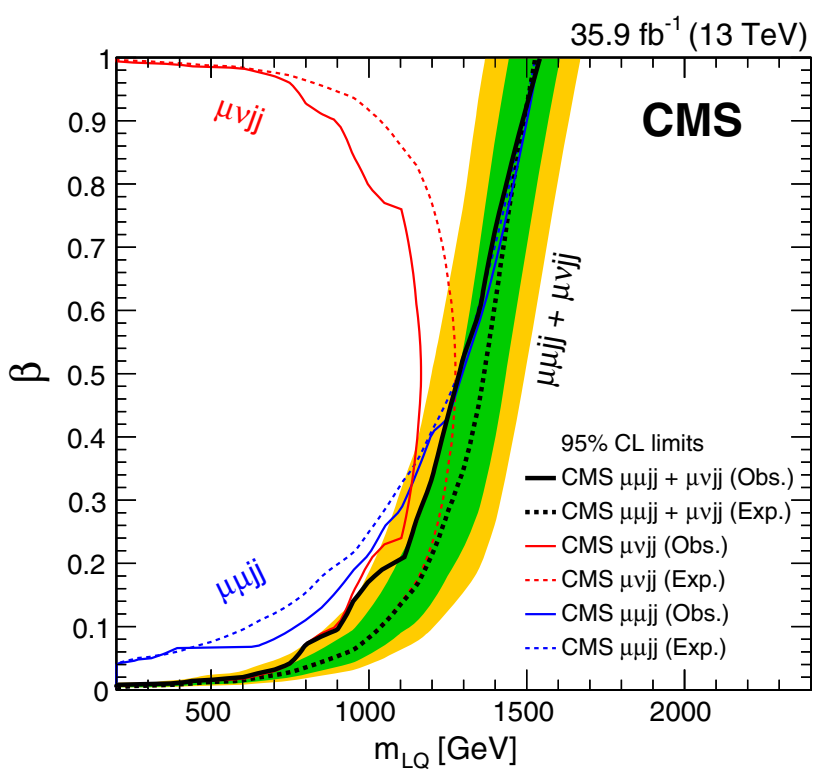

FIG. 9. The expected and observed exclusion limits at $95 \%$ C.L. for second-generation $m_{\mathrm{LQ}}$ as a function of the branching fraction $\beta$ vs. $m_{\mathrm{LQ}}$. The inner dark green and outer light yellow expected limit uncertainty bands represent the $68 \%$ and $95 \%$ confidence intervals on the combination. Limits for the individual $\mu \mu \mathrm{jj}$ and $\mu \nu \mathrm{jj}$ channels are also drawn. The solid lines represent the observed limits in each channel, and the dashed lines represent the expected limits.

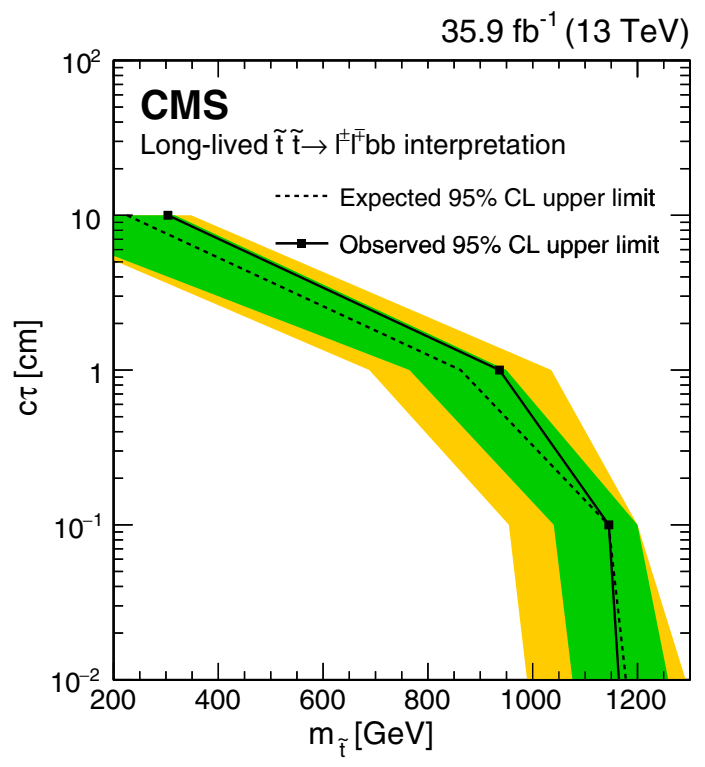

FIG. 10. Expected and observed upper limits at 95\% C.L. on the long-lived RPV SUSY $\tilde{t}$ pair production cross section as a function of $\tilde{t}$ mass ( $x$ axis) and lifetime ( $y$ axis). The dashed line and the inner dark green and outer light yellow uncertainty bands represent the median expected limits, and the $68 \%$ and $95 \%$ confidence intervals, respectively. angle between the left- and right-handed eigenstates of the top squarks. Using the expression for the partial width $\Gamma(\tilde{t} \rightarrow b \ell)=3 \Gamma(\tilde{t} \rightarrow b \mu) \approx 3 c\left(\lambda_{233}^{\prime} \cos (\theta)\right)^{2} m_{\tilde{t}} / 16 \pi$ [83], the excluded regions correspond to $\lambda_{233}^{\prime} \cos (\theta)<5.4 \times 10^{-8}$, $1.9 \times 10^{-8}$, and $1.0 \times 10^{-8}$, respectively, for the mass and lifetime limits described above. These limits provide complementary sensitivity to dedicated searches for long-lived particles [32], which generally require particles with longer decay lengths in their triggers.

\section{SUMMARY}

A search has been presented for pair production of second-generation leptoquarks using proton-proton collision data collected at $\sqrt{s}=13 \mathrm{TeV}$ in 2016 with the CMS detector at the LHC, corresponding to an integrated luminosity of $35.9 \mathrm{fb}^{-1}$. Limits are set at 95\% confidence level on the product of the scalar leptoquark pair production cross section and $\beta^{2}(2 \beta[1-\beta])$ in the $\mu \mu \mathrm{jj}(\mu \nu \mathrm{jj})$ channels, as a function of the leptoquark mass $m_{\mathrm{LQ}}$. Second-generation leptoquarks with masses less than $1530(1285) \mathrm{GeV}$ are excluded for $\beta=1.0(0.5)$, an improvement of 370 (525) GeV compared to previously published results. Two-dimensional limits are set in the $\beta-m_{\mathrm{LQ}}$ plane. The results in the $\mu \mu \mathrm{jj}$ search are interpreted in the context of an $R$-parity violating supersymmetry model with long-lived top squarks. These limits represent the most stringent limits to date on these models.

\section{ACKNOWLEDGMENTS}

We congratulate our colleagues in the CERN accelerator departments for the excellent performance of the LHC and thank the technical and administrative staffs at CERN and at other CMS institutes for their contributions to the success of the CMS effort. In addition, we gratefully acknowledge the computing centers and personnel of the Worldwide LHC Computing Grid for delivering so effectively the computing infrastructure essential to our analyses. Finally, we acknowledge the enduring support for the construction and operation of the LHC and the CMS detector provided by the following funding agencies: BMWFW and FWF (Austria); FNRS and FWO (Belgium); CNPq, CAPES, FAPERJ, and FAPESP (Brazil); MES (Bulgaria); CERN; CAS, MoST, and NSFC (China); COLCIENCIAS (Colombia); MSES and CSF (Croatia); RPF (Cyprus); SENESCYT (Ecuador); MoER, ERC IUT, and ERDF (Estonia); Academy of Finland, MEC, and HIP (Finland); CEA and CNRS/IN2P3 (France); BMBF, DFG, and HGF (Germany); GSRT (Greece); NKFIA (Hungary); DAE and DST (India); IPM (Iran); SFI (Ireland); INFN (Italy); MSIP and NRF (Republic of Korea); LAS (Lithuania); MOE and UM (Malaysia); BUAP, CINVESTAV, CONACYT, LNS, SEP, and UASLP-FAI (Mexico); MBIE (New Zealand); PAEC (Pakistan); MSHE and NSC (Poland); FCT (Portugal); JINR (Dubna); MON, RosAtom, RAS and RFBR (Russia); 
MESTD (Serbia); SEIDI, CPAN, PCTI and FEDER (Spain); Swiss Funding Agencies (Switzerland); MST (Taipei); ThEPCenter, IPST, STAR, and NSTDA (Thailand); TUBITAK and TAEK (Turkey); NASU and SFFR (Ukraine); STFC (United Kingdom); DOE and NSF (USA). Individuals have received support from the MarieCurie program and the European Research Council and Horizon 2020 Grant, Contract No. 675440 (European Union); the Leventis Foundation; the A.P. Sloan Foundation; the Alexander von Humboldt Foundation; the Belgian Federal Science Policy Office; the Fonds pour la Formation à la Recherche dans l'Industrie et dans l'Agriculture (FRIA-Belgium); the Agentschap voor Innovatie door Wetenschap en Technologie (IWTBelgium); the F. R. S.-FNRS and FWO (Belgium) under the "Excellence of Science-EOS"-be.h project no. 30820817; the Ministry of Education, Youth and Sports (MEYS) of the Czech Republic; the Lendület ("Momentum") Programme and the János Bolyai Research Scholarship of the Hungarian Academy of Sciences, the New National Excellence Program ÚNKP, the NKFIA research Grants
No. 123842 , No. 123959 , No. 124845 , No. 124850 and No. 125105 (Hungary); the Council of Science and Industrial Research, India; the HOMING PLUS program of the Foundation for Polish Science, cofinanced from European Union, Regional Development Fund, the Mobility Plus program of the Ministry of Science and Higher Education, the National Science Center (Poland), contracts Harmonia 2014/14/M/ST2/00428, Opus 2014/13/B/ST2/02543, 2014/ 15/B/ST2/03998, and 2015/19/B/ST2/02861, Sonata-bis 2012/07/E/ST2/01406; the National Priorities Research Program by Qatar National Research Fund; the Programa Estatal de Fomento de la Investigación Científica y Técnica de Excelencia María de Maeztu, Grant No. MDM-2015-0509 and the Programa Severo Ochoa del Principado de Asturias; the Thalis and Aristeia programs cofinanced by EU-ESF and the Greek NSRF; the Rachadapisek Sompot Fund for Postdoctoral Fellowship, Chulalongkorn University and the Chulalongkorn Academic into Its 2nd Century Project Advancement Project (Thailand); the Welch Foundation, Contract No. C-1845; and the Weston Havens Foundation (USA).

\section{APPENDIX: EFFICIENCIES AND EVENT YIELDS}

The product of signal acceptance and efficiency for optimized final selections as a function of $m_{\mathrm{LQ}}$ in the $\mu \mu \mathrm{jj}$ (left) and $\mu \nu \mathrm{jj}$ (right) channels is shown in Fig. 11. Event yields at final selection level for the $\mu \mu \mathrm{jj}$ and $\mu \nu \mathrm{jj}$ analyses are shown in Tables III and IV, respectively.
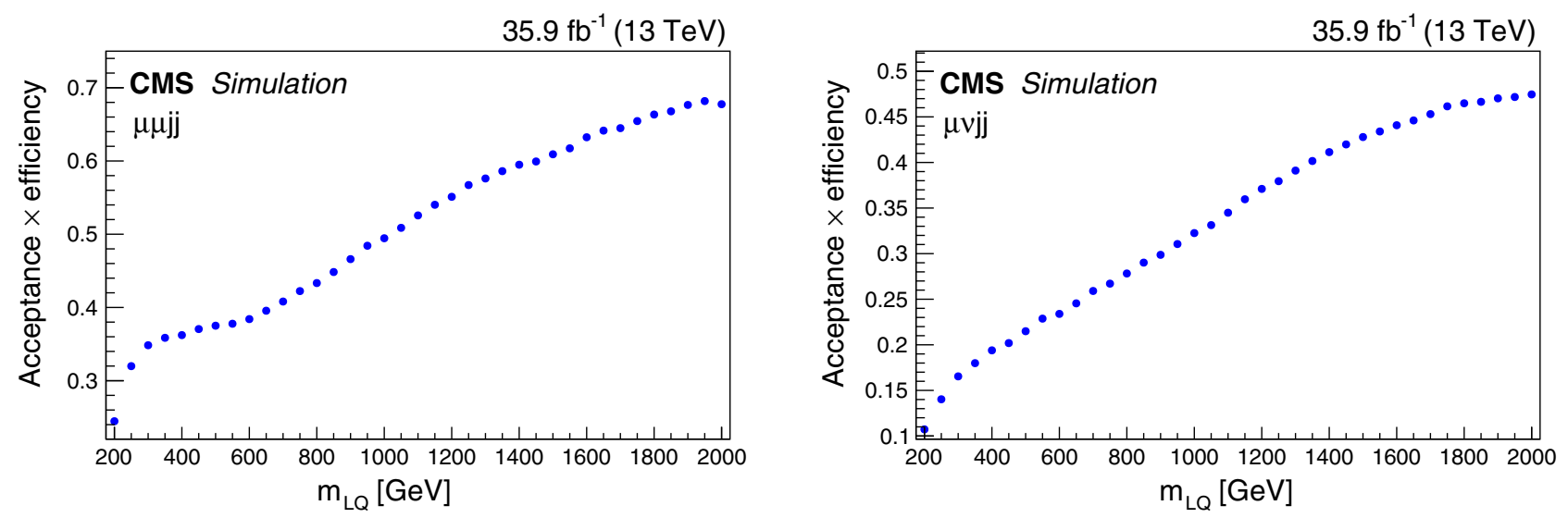

FIG. 11. The product of signal acceptance and efficiency for optimized final selections as a function of $m_{\mathrm{LQ}}$ in the $\mu \mu \mathrm{jj}$ (left) and $\mu \nu \mathrm{jj}$ (right) channels. 
TABLE III. Event yields after final selections for the $\mu \mu \mathrm{jj}$ analysis. "Other bkg." includes $W+$ jets and single top quark production. Uncertainties are statistical unless otherwise indicated.

\begin{tabular}{|c|c|c|c|c|c|c|c|}
\hline$m_{\mathrm{LQ}}(\mathrm{GeV})$ & Signal & $Z / \gamma^{*}+$ jets & $t \bar{t}+$ jets & Diboson & Other bkg. & All bkg. (stat + syst) & Data \\
\hline 200 & $531700 \pm 4700$ & $2973 \pm 7$ & $5467 \pm 56$ & $369 \pm 2$ & $519 \pm 10$ & $9328 \pm 57 \pm 444$ & 9317 \\
\hline 250 & $232900 \pm 1800$ & $1675 \pm 5$ & $2972 \pm 41$ & $241 \pm 2$ & $324 \pm 8$ & $5213 \pm 42 \pm 250$ & 5102 \\
\hline 300 & $100460 \pm 760$ & $793 \pm 3$ & $1298 \pm 26$ & $138 \pm 1$ & $189 \pm 6$ & $2419 \pm 27 \pm 117$ & 2360 \\
\hline 350 & $46160 \pm 340$ & $3878 \pm 2$ & $538 \pm 16$ & $81.0 \pm 1.0$ & $98.0 \pm 4.1$ & $1105 \pm 17 \pm 57$ & 1113 \\
\hline 400 & $22610 \pm 160$ & $202 \pm 1$ & $237 \pm 10$ & $51.9 \pm 0.8$ & $55.2 \pm 3.1$ & $546 \pm 11 \pm 29$ & 572 \\
\hline 450 & $12039 \pm 86$ & $132 \pm 1$ & $121 \pm 7$ & $32.2 \pm 0.7$ & $31.8 \pm 2.3$ & $316 \pm 78 \pm 18$ & 299 \\
\hline 500 & $6672 \pm 48$ & $79.0 \pm 0.7$ & $54.1 \pm 4.6$ & $20.9 \pm 0.5$ & $20.2 \pm 1.9$ & $174 \pm 5 \pm 11$ & 147 \\
\hline 550 & $3848 \pm 27$ & $52.0 \pm 0.5$ & $26.1 \pm 3.0$ & $14.4 \pm 0.5$ & $13.1 \pm 1.5$ & $106 \pm 3 \pm 8$ & 78 \\
\hline 600 & $2328 \pm 16$ & $34.7 \pm 0.4$ & $12.9 \pm 1.9$ & $10.0 \pm 0.3$ & $9.44 \pm 1.27$ & $67.0 \pm 2.4 \pm 5.2$ & 44 \\
\hline 650 & $1461 \pm 10$ & $26.0 \pm 0.3$ & $9.90 \pm 1.80$ & $6.55 \pm 0.30$ & $6.70 \pm 1.10$ & $49.0 \pm 2.1 \pm 3.9$ & 26 \\
\hline 700 & $948 \pm 7$ & $18.2 \pm 0.3$ & $4.68 \pm 1.07$ & $4.36 \pm 0.24$ & $4.53 \pm 0.91$ & $32.0 \pm 1.4 \pm 2.6$ & 16 \\
\hline 750 & $630 \pm 4$ & $12.4 \pm 0.2$ & $3.47 \pm 0.93$ & $3.17 \pm 0.20$ & $3.04 \pm 0.74$ & $22.0 \pm 1.2 \pm 1.9$ & 11 \\
\hline 800 & $424 \pm 3$ & $9.18 \pm 0.16$ & $2.62 \pm 0.83$ & $2.45 \pm 0.19$ & $2.26 \pm 0.63$ & $16.5 \pm 1.1 \pm 1.6$ & 8 \\
\hline 850 & $293 \pm 2$ & $6.93 \pm 0.13$ & $3.89 \pm 1.23$ & $1.88 \pm 0.17$ & $2.05 \pm 0.60$ & $14.8 \pm 1.4 \pm 1.1$ & 7 \\
\hline 900 & $206 \pm 1$ & $5.55 \pm 0.11$ & $2.34 \pm 0.88$ & $1.44 \pm 0.15$ & $1.49 \pm 0.50$ & $10.8 \pm 1.0 \pm 0.9$ & 6 \\
\hline 950 & $147 \pm 1$ & $4.41 \pm 0.10$ & $0.22 \pm 0.13$ & $1.31 \pm 0.15$ & $1.11 \pm 0.43$ & $7.04 \pm 0.48 \pm 0.71$ & 5 \\
\hline 1000 & $103.9 \pm 0.7$ & $3.66 \pm 0.09$ & $0.72 \pm 0.42$ & $1.10 \pm 0.13$ & $0.73 \pm 0.33$ & $6.21 \pm 0.56 \pm 0.59$ & 4 \\
\hline 1050 & $75.0 \pm 0.5$ & $3.23 \pm 0.09$ & $0.47 \pm 0.33$ & $0.93 \pm 0.12$ & $0.60 \pm 0.31$ & $5.24 \pm 0.48 \pm 0.56$ & 4 \\
\hline 1100 & $54.9 \pm 0.3$ & $2.71 \pm 0.07$ & $0.60 \pm 0.43$ & $0.69 \pm 0.10$ & $0.60 \pm 0.31$ & $4.60 \pm 0.54 \pm 0.48$ & 3 \\
\hline 1150 & $40.3 \pm 0.2$ & $2.39 \pm 0.07$ & $0.04 \pm 0.04$ & $0.69 \pm 0.10$ & $0.41 \pm 0.25$ & $3.53 \pm 0.28 \pm 0.42$ & 3 \\
\hline 1200 & $29.7 \pm 0.2$ & $1.86 \pm 0.06$ & $0.19 \pm 0.19$ & $0.63 \pm 0.10$ & $0.41 \pm 0.25$ & $3.10 \pm 0.33 \pm 0.42$ & 3 \\
\hline 1250 & $22.2 \pm 0.1$ & $1.68 \pm 0.06$ & $0.22 \pm 0.22$ & $0.56 \pm 0.10$ & $0.20 \pm 0.19$ & $2.65 \pm 0.31 \pm 0.34$ & 2 \\
\hline 1300 & $16.4 \pm 0.1$ & $1.13 \pm 0.04$ & $0.30 \pm 0.30$ & $0.53 \pm 0.10$ & $0.12 \pm 0.19$ & $2.15 \pm 0.37 \pm 0.27$ & 2 \\
\hline 1350 & $12.3 \pm 0.1$ & $1.26 \pm 0.05$ & $0.46 \pm 0.46$ & $0.53 \pm 0.10$ & $0.20 \pm 0.19$ & $2.45 \pm 0.51 \pm 0.24$ & 2 \\
\hline 1400 & $9.24 \pm 0.05$ & $1.14 \pm 0.04$ & $0.54 \pm 0.54$ & $0.54 \pm 0.11$ & $0.19_{-0.19}^{+0.28}$ & $2.41_{-0.59}^{+0.62} \pm 0.24$ & 2 \\
\hline 1450 & $6.90 \pm 0.04$ & $1.06 \pm 0.04$ & $0.58 \pm 0.58$ & $0.50 \pm 0.11$ & $0.19_{-0.19}^{+0.28}$ & $2.32_{-0.62}^{+0.65} \pm 0.22$ & 2 \\
\hline 1500 & $5.24 \pm 0.03$ & $1.05 \pm 0.05$ & $0.59 \pm 0.59$ & $0.47 \pm 0.11$ & $0.19_{-0.19}^{+0.28}$ & $2.30_{-0.63}^{+0.66} \pm 0.23$ & 2 \\
\hline 1550 & $3.99 \pm 0.02$ & $1.05 \pm 0.05$ & $0.59 \pm 0.59$ & $0.47 \pm 0.11$ & $0.19_{-0.19}^{+0.28}$ & $2.30_{-0.63}^{+0.66} \pm 0.23$ & 2 \\
\hline 1600 & $3.06 \pm 0.02$ & $1.05 \pm 0.05$ & $0.59 \pm 0.59$ & $0.47 \pm 0.11$ & $0.19_{-0.19}^{+0.28}$ & $2.30_{-0.63}^{+0.66} \pm 0.23$ & 2 \\
\hline 1650 & $2.35 \pm 0.01$ & $1.05 \pm 0.05$ & $0.59 \pm 0.59$ & $0.47 \pm 0.11$ & $0.19_{-0.19}^{+0.28}$ & $2.30_{-0.63}^{+0.66} \pm 0.23$ & 2 \\
\hline 1700 & $1.79 \pm 0.01$ & $1.05 \pm 0.05$ & $0.59 \pm 0.59$ & $0.47 \pm 0.11$ & $0.19_{-0.19}^{+0.28}$ & $2.30_{-0.63}^{+0.66} \pm 0.23$ & 2 \\
\hline 1750 & $1.38 \pm 0.01$ & $1.05 \pm 0.05$ & $0.59 \pm 0.59$ & $0.47 \pm 0.11$ & $0.19_{-0.19}^{+0.28}$ & $2.30_{-0.63}^{+0.66} \pm 0.23$ & 2 \\
\hline 1800 & $1.07 \pm 0.01$ & $1.05 \pm 0.05$ & $0.59 \pm 0.59$ & $0.47 \pm 0.11$ & $0.19_{-0.19}^{+0.28}$ & $2.30_{-0.63}^{+0.66} \pm 0.23$ & 2 \\
\hline 1850 & $0.821 \pm 0.004$ & $1.05 \pm 0.05$ & $0.59 \pm 0.59$ & $0.47 \pm 0.11$ & $0.19_{-0.19}^{+0.28}$ & $2.30_{-0.63}^{+0.66} \pm 0.23$ & 2 \\
\hline 1900 & $0.636 \pm 0.003$ & $1.05 \pm 0.05$ & $0.59 \pm 0.59$ & $0.47 \pm 0.11$ & $0.19_{-0.19}^{+0.28}$ & $2.30_{-0.63}^{+0.66} \pm 0.23$ & 2 \\
\hline 1950 & $0.491 \pm 0.003$ & $1.05 \pm 0.05$ & $0.59 \pm 0.59$ & $0.47 \pm 0.11$ & $0.19_{-0.19}^{+0.28}$ & $2.30_{-0.63}^{+0.66} \pm 0.23$ & 2 \\
\hline 2000 & $0.377 \pm 0.002$ & $1.05 \pm 0.05$ & $0.59 \pm 0.59$ & $0.47 \pm 0.11$ & $0.19_{-0.19}^{+0.28}$ & $2.30_{-0.63}^{+0.66} \pm 0.23$ & 2 \\
\hline
\end{tabular}

TABLE IV. Event yields after final selections for the $\mu \nu \mathrm{jj}$ analysis. "Other bkg." includes $Z / \gamma^{*}+$ jets and single top quark production. Uncertainties are statistical unless otherwise indicated.

\begin{tabular}{|c|c|c|c|c|c|c|c|}
\hline$m_{\mathrm{LQ}}(\mathrm{GeV})$ & Signal & $W+$ jets & $t \bar{t}+$ jets & Diboson & Other bkg. & All bkg. (stat + syst) & Data \\
\hline 200 & $116600 \pm 1500$ & $5672 \pm 26$ & $15816 \pm 51$ & $1049 \pm 5$ & $2732 \pm 15$ & $25270 \pm 59 \pm 1171$ & 26043 \\
\hline 250 & $51050 \pm 580$ & $2635 \pm 16$ & $4662 \pm 28$ & $575 \pm 3$ & $1155 \pm 10$ & $9029 \pm 34 \pm 431$ & 9519 \\
\hline 300 & $23840 \pm 250$ & $1259 \pm 10$ & $2066 \pm 18$ & $346 \pm 3$ & $611.7 \pm 7$ & $4284 \pm 22 \pm 197$ & 4669 \\
\hline 350 & $11580 \pm 120$ & $757 \pm 7$ & $964 \pm 13$ & $200 \pm 2$ & $335 \pm 5$ & $2256 \pm 16 \pm 122$ & 2379 \\
\hline 400 & $6051 \pm 58$ & $418 \pm 5$ & $461 \pm 9$ & $131 \pm 2$ & $176 \pm 4$ & $1187 \pm 11 \pm 70$ & 1279 \\
\hline 450 & $3280 \pm 32$ & $248 \pm 3$ & $228 \pm 6$ & $86.4 \pm 1.6$ & $108 \pm 3$ & $671 \pm 8 \pm 47$ & 737 \\
\hline 500 & $1911 \pm 18$ & $177 \pm 3$ & $119 \pm 4$ & $58.8 \pm 1.3$ & $67.6 \pm 2.7$ & $422 \pm 6 \pm 40$ & 430 \\
\hline
\end{tabular}


TABLE IV. (Continued)

\begin{tabular}{|c|c|c|c|c|c|c|c|}
\hline$m_{\mathrm{LQ}}(\mathrm{GeV})$ & Signal & $W+$ jets & $t \bar{t}+$ jets & Diboson & Other bkg. & All bkg. (stat + syst) & Data \\
\hline 550 & $1165 \pm 10$ & $99.2 \pm 1.8$ & $69.2 \pm 3.4$ & $44.0 \pm 1.2$ & $42.9 \pm 2.1$ & $255 \pm 4 \pm 19$ & 270 \\
\hline 600 & $7089 \pm 6$ & $70.9 \pm 1.5$ & $43.4 \pm 2.7$ & $31.1 \pm 1.0$ & $28.6 \pm 1.7$ & $174 \pm 3 \pm 13$ & 179 \\
\hline 650 & $453 \pm 4$ & $53.8 \pm 1.3$ & $26.8 \pm 2.1$ & $22.9 \pm 0.91$ & $19.7 \pm 1.4$ & $123 \pm 3 \pm 10$ & 130 \\
\hline 700 & $301 \pm 3$ & $36.0 \pm 1.9$ & $16.7 \pm 1.7$ & $17.0 \pm 0.78$ & $14.8 \pm 1.2$ & $84.6 \pm 2.4 \pm 7.1$ & 93 \\
\hline 750 & $199 \pm 2$ & $22.7 \pm 0.7$ & $11.6 \pm 1.4$ & $13.3 \pm 0.71$ & $9.89 \pm 0.96$ & $57.5 \pm 2.0 \pm 5.2$ & 68 \\
\hline 800 & $136 \pm 1$ & $14.0 \pm 0.5$ & $7.60 \pm 1.15$ & $8.58 \pm 0.52$ & $7.60 \pm 0.83$ & $37.7 \pm 1.6 \pm 4.3$ & 57 \\
\hline 850 & $94.7 \pm 0.8$ & $10.5 \pm 0.4$ & $4.88 \pm 0.92$ & $7.46 \pm 0.52$ & $6.51 \pm 0.81$ & $29.3 \pm 1.4 \pm 3.5$ & 45 \\
\hline 900 & $65.9 \pm 0.5$ & $8.96 \pm 0.34$ & $3.43 \pm 0.79$ & $6.14 \pm 0.48$ & $5.56 \pm 0.75$ & $24.1 \pm 1.2 \pm 2.4$ & 35 \\
\hline 950 & $47.1 \pm 0.4$ & $5.96 \pm 0.25$ & $2.36 \pm 0.65$ & $4.85 \pm 0.42$ & $3.70 \pm 0.55$ & $16.9 \pm 1.0 \pm 1.7$ & 30 \\
\hline 1000 & $33.9 \pm 0.3$ & $5.40 \pm 0.24$ & $1.66 \pm 0.55$ & $4.30 \pm 0.41$ & $3.30 \pm 0.52$ & $14.7 \pm 0.9 \pm 1.5$ & 26 \\
\hline 1050 & $24.4 \pm 0.2$ & $4.20 \pm 0.20$ & $1.48 \pm 0.52$ & $3.90 \pm 0.40$ & $2.54 \pm 0.45$ & $12.1 \pm 0.8 \pm 1.3$ & 20 \\
\hline 1100 & $18.0 \pm 0.1$ & $4.16 \pm 0.22$ & $1.29 \pm 0.49$ & $3.31 \pm 0.38$ & $1.83 \pm 0.33$ & $10.6 \pm 0.7 \pm 1.2$ & 15 \\
\hline 1150 & $13.4 \pm 0.1$ & $3.05 \pm 0.17$ & $0.76 \pm 0.38$ & $2.87 \pm 0.35$ & $1.29 \pm 0.28$ & $7.97 \pm 0.61 \pm 0.92$ & 13 \\
\hline 1200 & $9.98 \pm 0.07$ & $3.02 \pm 0.18$ & $0.56 \pm 0.32$ & $2.29 \pm 0.31$ & $1.09 \pm 0.23$ & $6.96 \pm 0.54 \pm 0.81$ & 11 \\
\hline 1250 & $7.42 \pm 0.05$ & $2.68 \pm 0.17$ & $0.74 \pm 0.37$ & $2.07 \pm 0.30$ & $0.59 \pm 0.14$ & $6.08 \pm 0.52 \pm 0.72$ & 11 \\
\hline 1300 & $5.58 \pm 0.04$ & $1.61 \pm 0.11$ & $0.74 \pm 0.37$ & $1.79 \pm 0.28$ & $0.73 \pm 0.14$ & $4.87 \pm 0.49 \pm 0.55$ & 9 \\
\hline 1350 & $4.21 \pm 0.03$ & $1.03 \pm 0.07$ & $0.74 \pm 0.37$ & $1.50 \pm 0.25$ & $0.70 \pm 0.14$ & $3.97 \pm 0.48 \pm 0.43$ & 7 \\
\hline 1400 & $3.19 \pm 0.02$ & $1.01 \pm 0.08$ & $0.74 \pm 0.37$ & $1.33 \pm 0.26$ & $0.69 \pm 0.14$ & $3.76 \pm 0.48 \pm 0.39$ & 7 \\
\hline 1450 & $2.42 \pm 0.02$ & $1.45 \pm 0.12$ & $0.56 \pm 0.32$ & $1.32 \pm 0.26$ & $0.65 \pm 0.14$ & $3.97 \pm 0.45 \pm 0.44$ & 7 \\
\hline 1500 & $1.84 \pm 0.01$ & $1.29 \pm 0.11$ & $0.56 \pm 0.32$ & $1.32 \pm 0.26$ & $0.58 \pm 0.14$ & $3.75 \pm 0.45 \pm 0.41$ & 7 \\
\hline 1550 & $1.40 \pm 0.01$ & $1.12 \pm 0.10$ & $0.56 \pm 0.32$ & $1.32 \pm 0.26$ & $0.49 \pm 0.14$ & $3.49 \pm 0.45 \pm 0.39$ & 6 \\
\hline 1600 & $1.07 \pm 0.01$ & $1.07 \pm 0.10$ & $0.56 \pm 0.32$ & $1.27 \pm 0.26$ & $0.46 \pm 0.14$ & $3.35 \pm 0.45 \pm 0.37$ & 6 \\
\hline 1650 & $0.82 \pm 0.01$ & $0.88 \pm 0.09$ & $0.56 \pm 0.32$ & $1.27 \pm 0.26$ & $0.44 \pm 0.14$ & $3.15 \pm 0.44 \pm 0.35$ & 6 \\
\hline 1700 & $0.629 \pm 0.004$ & $0.99 \pm 0.11$ & $0.56 \pm 0.32$ & $1.05 \pm 0.24$ & $0.42 \pm 0.14$ & $3.01 \pm 0.44 \pm 0.32$ & 6 \\
\hline 1750 & $0.487 \pm 0.003$ & $0.91 \pm 0.11$ & $0.38 \pm 0.27$ & $0.98 \pm 0.23$ & $0.38 \pm 0.14$ & $2.65 \pm 0.39 \pm 0.30$ & 5 \\
\hline 1800 & $0.373 \pm 0.002$ & $0.91 \pm 0.11$ & $0.38 \pm 0.27$ & $0.96 \pm 0.24$ & $0.36 \pm 0.14$ & $2.61 \pm 0.40 \pm 0.29$ & 5 \\
\hline 1850 & $0.287 \pm 0.002$ & $0.88 \pm 0.11$ & $0.20 \pm 0.20$ & $0.90 \pm 0.23$ & $0.32 \pm 0.14$ & $2.30 \pm 0.35 \pm 0.28$ & 4 \\
\hline 1900 & $0.221 \pm 0.001$ & $0.74 \pm 0.10$ & $0.20 \pm 0.20$ & $0.86 \pm 0.24$ & $0.31 \pm 0.14$ & $2.11 \pm 0.35 \pm 0.25$ & 3 \\
\hline 1950 & $0.170 \pm 0.001$ & $0.69 \pm 0.10$ & $0.20 \pm 0.20$ & $0.83 \pm 0.24$ & $0.30 \pm 0.14$ & $2.02 \pm 0.35 \pm 0.24$ & 3 \\
\hline 2000 & $0.132 \pm 0.001$ & $0.68 \pm 0.10$ & $0.29 \pm 0.20$ & $0.29 \pm 0.09$ & $0.30 \pm 0.14$ & $1.47 \pm 0.28 \pm 0.15$ & 2 \\
\hline
\end{tabular}

[1] J. C. Pati and A. Salam, Unified lepton-hadron symmetry and a gauge theory of the basic interactions, Phys. Rev. D 8, 1240 (1973).

[2] J. C. Pati and A. Salam, Lepton number as the fourth color, Phys. Rev. D 10, 275 (1974).

[3] H. Georgi and S. Glashow, Unity of All Elementary-Particle Forces, Phys. Rev. Lett. 32, 438 (1974).

[4] H. Murayama and T. Yanagida, A viable SU(5) GUT with light leptoquark bosons, Mod. Phys. Lett. A 07, 147 (1992).

[5] H. Fritzsch and P. Minkowski, United interactions of leptons and hadrons, Ann. Phys. (N.Y.) 93, 193 (1975).

[6] G. Senjanović and A. Sokorac, Light lepto-quarks in $\mathrm{SO}(10)$, Z. Phys. C 20, 255 (1983).

[7] P. H. Frampton and B.-H. Lee, SU(15) Grand Unification, Phys. Rev. Lett. 64, 619 (1990).

[8] P. H. Frampton and T. W. Kephart, Higgs sector and proton decay in SU(15q) grand unification, Phys. Rev. D 42, 3892 (1990).

[9] B. Schrempp and F. Schrempp, Light leptoquarks, Phys. Lett. 153B, 101 (1985).
[10] S. Dimopoulos and L. Susskind, Mass without scalars, Nucl. Phys. B155, 237 (1979).

[11] S. Dimopoulos, Technicolored signatures, Nucl. Phys. B168, 69 (1980).

[12] E. Eichten and K. Lane, Dynamical breaking of the weak interaction symmetries, Phys. Lett. 90B, 85 (1980).

[13] J. L. Hewett and T. G. Rizzo, Low-energy phenomenology of superstring-inspired $\mathrm{E}_{6}$ models, Phys. Rep. 183, 193 (1989).

[14] W. Buchmüller, R. Rückl, and D. Wyler, Leptoquarks in lepton-quark collisions, Phys. Lett. B 191, 442 (1987).

[15] A. Matyja et al. (Belle Collaboration), Observation of $B^{0} \rightarrow$ $D^{*-} \tau^{+} \nu_{\tau}$ Decay at Belle, Phys. Rev. Lett. 99, 191807 (2007).

[16] A. Bozek et al. (Belle Collaboration), Observation of $B^{+} \rightarrow \bar{D}^{* 0} \tau^{+} \nu_{\tau}$ and evidence for $b^{+} \rightarrow \bar{D}^{0} \tau^{+} \nu_{\tau}$ at Belle, Phys. Rev. D 82, 072005 (2010).

[17] M. Huschle et al. (Belle Collaboration), Measurement of the branching ratio of $\bar{B} \rightarrow D^{(*)} \tau^{-} \bar{\nu}_{\tau}$ relative to $\bar{B} \rightarrow D^{(*)} \ell^{-} \bar{\nu}_{\ell}$ decays with hadronic tagging at Belle, Phys. Rev. D 92, 072014 (2015). 
[18] J. P. Lees et al. (BABAR Collaboration), Evidence for an Excess of $\bar{B} \rightarrow D^{(*)} \tau^{-} \bar{\nu}_{\tau}$ Decays, Phys. Rev. Lett. 109, 101802 (2012).

[19] J. P. Lees et al. (BABAR Collaboration), Measurement of an excess of $\bar{B} \rightarrow D^{(*)} \tau^{-} \bar{\nu}_{\tau}$ decays and implications for charged Higgs bosons, Phys. Rev. D 88, 072012 (2013).

[20] LHCb Collaboration, Measurement of the Ratio of Branching Fractions $\mathcal{B}\left(\bar{B}^{0} \rightarrow D^{*+} \tau^{-} \bar{\nu}_{\tau}\right) / \mathcal{B}\left(\bar{B}^{0} \rightarrow D^{*+} \mu^{-} \bar{\nu}_{\mu}\right)$, Phys. Rev. Lett. 115, 111803 (2015); 115, 159901(E) (2015).

[21] LHCb Collaboration, Measurement of Form-Factor-Independent Observables in the Decay $B^{0} \rightarrow K^{* 0} \mu^{+} \mu^{-}$, Phys. Rev. Lett. 111, 191801 (2013).

[22] LHCb Collaboration, Test of Lepton Universality Using $B^{+} \rightarrow K^{+} \ell^{+} \ell^{-}$Decays, Phys. Rev. Lett. 113, 151601 (2014).

[23] LHCb Collaboration, Test of lepton universality with $B^{0} \rightarrow$ $K^{* 0} \ell^{+} \ell^{-}$decays, J. High Energy Phys. 08 (2017) 055.

[24] J. Blumlein, E. Boos, and A. Kryukov, Leptoquark pair production in hadronic interactions, Z. Phys. C 76, 137 (1997).

[25] B. Diaz, M. Schmaltz, and Y.-M. Zhong, The leptoquark hunter's guide: Pair production, J. High Energy Phys. 10 (2017) 097.

[26] W. Buchmüller and D. Wyler, Constraints on SU(5)-type leptoquarks, Phys. Lett. B 177, 377 (1986).

[27] O. Shanker, $\pi \ell 2, \mathrm{~K} \ell 3$, and $\mathrm{K}^{0}-\overline{\mathrm{K}}^{0}$ constraints on leptoquarks and supersymmetric particles, Nucl. Phys. B204, 375 (1982).

[28] CMS Collaboration, Search for pair production of first and second generation leptoquarks in proton-proton collisions at $\sqrt{s}=8 \mathrm{TeV}$, Phys. Rev. D 93, 032004 (2016).

[29] ATLAS Collaboration, Search for scalar leptoquarks in $p p$ collisions at $\sqrt{s}=13 \mathrm{TeV}$ with the ATLAS experiment, New J. Phys. 18, 093016 (2016).

[30] H. K. Dreiner, An introduction to explicit $R$-parity violation, Pramana 51, 123 (1998).

[31] P. W. Graham, D. E. Kaplan, S. Rajendran, and P. Saraswat, Displaced supersymmetry, J. High Energy Phys. 07 (2012) 149.

[32] CMS Collaboration, Search for Displaced Supersymmetry in Events with an Electron and a Muon with Large Impact Parameters, Phys. Rev. Lett. 114, 061801 (2015).

[33] CMS Collaboration, The CMS experiment at the CERN LHC, J. Instrum. 3, S08004 (2008).

[34] CMS Collaboration, The CMS trigger system, J. Instrum. 12, P01020 (2017).

[35] CMS Collaboration, CMS luminosity measurements for the 2016 data-taking period, Technical Report No. CMS-PASLUM-17-001, 2017, https://cds.cern.ch/record/2257069.

[36] T. Sjöstrand, S. Ask, J. R. Christiansen, R. Corke, N. Desai, P. Ilten, S. Mrenna, S. Prestel, C. O. Rasmussen, and P. Z. Skands, An introduction to PYTHIA 8.2, Comput. Phys. Commun. 191, 159 (2015).

[37] M. Krämer, T. Plehn, M. Spira, and P. M. Zerwas, Pair production of scalar leptoquarks at the CERN LHC, Phys. Rev. D 71, 057503 (2005).

[38] J. Pumplin, D. R. Stump, J. Huston, H. L. Lai, P. Nadolsky, and W. K. Tung, New generation of parton distributions with uncertainties from global QCD analysis, J. High Energy Phys. 07 (2002) 012.
[39] P. M. Nadolsky, H.-L. Lai, Q.-H. Cao, J. Huston, J. Pumplin, D. Stump, W.-K. Tung, and C. P. Yuan, Implications of CTEQ global analysis for collider observables, Phys. Rev. D 78, 013004 (2008).

[40] B. C. Allanach et al., The Snowmass Points and Slopes: benchmarks for SUSY searches, Eur. Phys. J. C 25, 113 (2002).

[41] W. Beenakker, M. Krämer, T. Plehn, M. Spira, and P. M. Zerwas, Stop production at hadron colliders, Nucl. Phys. B515, 3 (1998).

[42] W. Beenakker, S. Brensing, M. Krämer, A. Kulesza, E. Laenen, and I. Niessen, Supersymmetric top and bottom squark production at hadron colliders, J. High Energy Phys. 08 (2010) 098.

[43] W. Beenakker, S. Brensing, M. Krämer, A. Kulesza, E. Laenen, L. Motyka, and I. Niessen, Squark and gluino hadroproduction, Int. J. Mod. Phys. A 26, 2637 (2011).

[44] J. Alwall, R. Frederix, S. Frixione, V. Hirschi, F. Maltoni, O. Mattelaer, H. S. Shao, T. Stelzer, P. Torrielli, and M. Zaro, The automated computation of tree-level and next-toleading order differential cross sections, and their matching to parton shower simulations, J. High Energy Phys. 07 (2014) 079.

[45] S. Frixione and B. R. Webber, Matching NLO QCD computations and parton shower simulations, J. High Energy Phys. 06 (2002) 029.

[46] S. Frixione, P. Nason, and C. Oleari, Matching NLO QCD computations with parton shower simulations: The POWHEG method, J. High Energy Phys. 11 (2007) 070.

[47] S. Alioli, P. Nason, C. Oleari, and E. Re, A general framework for implementing NLO calculations in shower Monte Carlo programs: The POWHEG BOX, J. High Energy Phys. 06 (2010) 043.

[48] S. Alioli, P. Nason, C. Oleari, and E. Re, NLO vector-boson production matched with shower in POWHEG, J. High Energy Phys. 07 (2008) 060.

[49] S. Alioli, P. Nason, C. Oleari, and E. Re, NLO single-top production matched with shower in POWHEG: $s$ - and $t$-channel contributions, J. High Energy Phys. 09 (2009) 111; 02 (2010) 11.

[50] S. Frixione, E. Laenen, P. Motylinski, and B. R. Webber, Single-top production in MC@NLO, J. High Energy Phys. 03 (2006) 092.

[51] Y. Li and F. Petriello, Combining QCD and electroweak corrections to dilepton production in FEWZ, Phys. Rev. D 86, 094034 (2012).

[52] J. Campbell, R. K. Ellis, and F. Tramontano, Single topquark production and decay at next-to-leading order, Phys. Rev. D 70, 094012 (2004).

[53] J. Campbell and F. Tramontano, Next-to-leading order corrections to Wt production and decay, Nucl. Phys. B726, 109 (2005).

[54] J. M. Campbell, R. Frederix, F. Maltoni, and F. Tramontano, Next-to-Leading-Order Predictions for $t$-Channel SingleTop Production at Hadron Colliders, Phys. Rev. Lett. 102, 182003 (2009).

[55] J. M. Campbell, R. Keith Ellis, and C. Williams, Vector boson pair production at the LHC, J. High Energy Phys. 07 (2011) 018. 
[56] M. Beneke, P. Falgari, S. Klein, and C. Schwinn, Hadronic top-quark pair production with NNLL threshold resummation, Nucl. Phys. B855, 695 (2012).

[57] M. Cacciari, M. Czakon, M. Mangano, A. Mitov, and P. Nason, Top-pair production at hadron colliders with next-tonext-to-leading logarithmic soft-gluon resummation, Phys. Lett. B 710, 612 (2012).

[58] P. Bärnreuther, M. Czakon, and A. Mitov, Percent Level Precision Physics at the Tevatron: First Genuine NNLO QCD Corrections to $q \bar{q} \rightarrow t \bar{t}+X$, Phys. Rev. Lett. 109, 132001 (2012).

[59] M. Czakon and A. Mitov, NNLO corrections to top-pair production at hadron colliders: The all-fermionic scattering channels, J. High Energy Phys. 12 (2012) 054.

[60] M. Czakon and A. Mitov, NNLO corrections to top pair production at hadron colliders: The quark-gluon reaction, J. High Energy Phys. 01 (2013) 080.

[61] M. Czakon, P. Fiedler, and A. Mitov, Total Top-Quark PairProduction Cross Section at Hadron Colliders Through $O\left(\alpha_{S}^{4}\right)$, Phys. Rev. Lett. 110, 252004 (2013).

[62] M. Czakon and A. Mitov, Top++: A program for the calculation of the top-pair cross-section at hadron colliders, Comput. Phys. Commun. 185, 2930 (2014).

[63] R. D. Ball et al. (NNPDF Collaboration), Parton distributions for the LHC Run II, J. High Energy Phys. 04 (2015) 040.

[64] S. Agostinelli et al. (GEANT4 Collaboration), GEANT4-A simulation toolkit, Nucl. Instrum. Methods Phys. Res., Sect. A 506, 250 (2003).

[65] J. Allison et al., GEANT4 developments and applications, IEEE Trans. Nucl. Sci. 53, 270 (2006).

[66] CMS Collaboration, Event generator tunes obtained from underlying event and multiparton scattering measurements, Eur. Phys. J. C 76, 155 (2016).

[67] CMS Collaboration, Particle-flow reconstruction and global event description with the CMS detector, J. Instrum. 12, P10003 (2017).

[68] M. Cacciari, G. P. Salam, and G. Soyez, The anti- $k_{\mathrm{T}}$ jet clustering algorithm, J. High Energy Phys. 08 (2008) 063.

[69] M. Cacciari, G. P. Salam, and G. Soyez, FastJet user manual, Eur. Phys. J. C 72, 1896 (2012).
[70] CMS Collaboration, Jet energy scale and resolution in the CMS experiment in pp collisions at $8 \mathrm{TeV}$, J. Instrum. 12, P02014 (2017).

[71] CMS Collaboration, Identification of heavy-flavour jets with the CMS detector in pp collisions at $13 \mathrm{TeV}$, J. Instrum. 13, P05011 (2018).

[72] CMS Collaboration, Performance of the CMS muon detector and muon reconstruction with proton-proton collisions at $\sqrt{s}=13 \mathrm{TeV}$, J. Instrum. 13, P06015 (2018).

[73] CMS Collaboration, Performance of electron reconstruction and selection with the CMS detector in proton-proton collisions at $\sqrt{s}=8 \mathrm{TeV}$, J. Instrum. 10, P06005 (2015).

[74] G. Punzi, Sensitivity of searches for new signals and its optimization, eConf C030908, MODT002 (2003).

[75] CMS Collaboration, Search for high-mass resonances in dilepton final states in proton-proton collisions at $\sqrt{s}=13 \mathrm{TeV}$, J. High Energy Phys. 06 (2018) 120.

[76] CMS Collaboration, Measurement of the inelastic protonproton cross section at $\sqrt{s}=13 \mathrm{TeV}$, J. High Energy Phys. 07 (2018) 161.

[77] M. Botje et al., The PDF4LHC working group interim recommendations, arXiv:1101.0538.

[78] D. Bourilkov, R. C. Group, and M. R. Whalley, LHAPDF: PDF use from the Tevatron to the LHC, arXiv:hep-ph/ 0605240 .

[79] G. Cowan, K. Cranmer, E. Gross, and O. Vitells, Asymptotic formulae for likelihood-based tests of new physics, Eur. Phys. J. C 71, 1554 (2011); 73, 2501(E) (2013).

[80] T. Junk, Confidence level computation for combining searches with small statistics, Nucl. Instrum. Methods Phys. Res., Sect. A 434, 435 (1999).

[81] A. L. Read, Presentation of search results: The $\mathrm{CL}_{\mathrm{s}}$ technique, J. Phys. G 28, 2693 (2002).

[82] ATLAS and CMS Collaborations, Procedure for the LHC Higgs boson search combination in summer 2011, Technical Reports No. CMS-NOTE-2011-005 and No. ATL-PHYSPUB-2011-11, 2011.

[83] P. Richardson, Simulations of $R$-parity violating SUSY models, Ph.D. thesis, Oxford University, 2000, arXiv: hep-ph/0101105.

A. M. Sirunyan, ${ }^{1}$ A. Tumasyan, ${ }^{1}$ W. Adam, ${ }^{2}$ F. Ambrogi, ${ }^{2}$ E. Asilar, ${ }^{2}$ T. Bergauer, ${ }^{2}$ J. Brandstetter, ${ }^{2}$ M. Dragicevic, ${ }^{2}$ J. Erö, ${ }^{2}$ A. Escalante Del Valle, ${ }^{2}$ M. Flechl, ${ }^{2}$ R. Frühwirth, ${ }^{2, b}$ V. M. Ghete, ${ }^{2}$ J. Hrubec, ${ }^{2}$ M. Jeitler, ${ }^{2, b}$ N. Krammer, ${ }^{2}$ I. Krätschmer, ${ }^{2}$ D. Liko, ${ }^{2}$ T. Madlener, ${ }^{2}$ I. Mikulec, ${ }^{2}$ N. Rad, ${ }^{2}$ H. Rohringer, ${ }^{2}$ J. Schieck, ${ }^{2, b}$ R. Schöfbeck, ${ }^{2}$ M. Spanring, ${ }^{2}$ D. Spitzbart, ${ }^{2}$ A. Taurok, ${ }^{2}$ W. Waltenberger, ${ }^{2}$ J. Wittmann, ${ }^{2}$ C.-E. Wulz, ${ }^{2, b}$ M. Zarucki, ${ }^{2}$ V. Chekhovsky, ${ }^{3}$ V. Mossolov, ${ }^{3}$ J. Suarez Gonzalez, ${ }^{3}$ E. A. De Wolf, ${ }^{4}$ D. Di Croce, ${ }^{4}$ X. Janssen, ${ }^{4}$ J. Lauwers, ${ }^{4}$ M. Pieters, ${ }^{4}$ H. Van Haevermaet,${ }^{4}$ P. Van Mechelen, ${ }^{4}$ N. Van Remortel, ${ }^{4}$ S. Abu Zeid, ${ }^{5}$ F. Blekman, ${ }^{5}$ J. D’Hondt, ${ }^{5}$ I. De Bruyn, ${ }^{5}$ J. De Clercq, ${ }^{5}$ K. Deroover, ${ }^{5}$ G. Flouris, ${ }^{5}$ D. Lontkovskyi, ${ }^{5}$ S. Lowette, ${ }^{5}$ I. Marchesini, ${ }^{5}$ S. Moortgat,${ }^{5}$ L. Moreels, ${ }^{5}$ Q. Python, ${ }^{5}$ K. Skovpen, ${ }^{5}$ S. Tavernier, ${ }^{5}$ W. Van Doninck, ${ }^{5}$ P. Van Mulders, ${ }^{5}$ I. Van Parijs, ${ }^{5}$ D. Beghin, ${ }^{6}$ B. Bilin, ${ }^{6}$ H. Brun, ${ }^{6}$ B. Clerbaux, ${ }^{6}$ G. De Lentdecker, ${ }^{6}$ H. Delannoy, ${ }^{6}$ B. Dorney, ${ }^{6}$ G. Fasanella, ${ }^{6}$ L. Favart, ${ }^{6}$ R. Goldouzian, ${ }^{6}$ A. Grebenyuk, ${ }^{6}$ A. K. Kalsi, ${ }^{6}$ T. Lenzi, ${ }^{6}$ J. Luetic, ${ }^{6}$ N. Postiau, ${ }^{6}$ E. Starling, ${ }^{6}$ L. Thomas, ${ }^{6}$ C. Vander Velde,${ }^{6}$ P. Vanlaer, ${ }^{6}$ D. Vannerom, ${ }^{6}$ Q. Wang, ${ }^{6}$ T. Cornelis, ${ }^{7}$ D. Dobur, ${ }^{7}$ A. Fagot, ${ }^{7}$ M. Gul, ${ }^{7}$ I. Khvastunov, ${ }^{7, c}$ D. Poyraz, ${ }^{7}$ C. Roskas, ${ }^{7}$ D. Trocino, ${ }^{7}$ M. Tytgat, ${ }^{7}$ W. Verbeke, ${ }^{7}$ B. Vermassen, ${ }^{7}$ M. Vit, ${ }^{7}$ N. Zaganidis, ${ }^{7}$ H. Bakhshiansohi, ${ }^{8}$ O. Bondu, ${ }^{8}$ S. Brochet, ${ }^{8}$ G. Bruno, ${ }^{8}$ C. Caputo, ${ }^{8}$ 
P. David,${ }^{8}$ C. Delaere,${ }^{8}$ M. Delcourt, ${ }^{8}$ A. Giammanco,${ }^{8}$ G. Krintiras,${ }^{8}$ V. Lemaitre, ${ }^{8}$ A. Magitteri, ${ }^{8}$ A. Mertens, ${ }^{8}$ K. Piotrzkowski, ${ }^{8}$ A. Saggio, ${ }^{8}$ M. Vidal Marono, ${ }^{8}$ S. Wertz, ${ }^{8}$ J. Zobec, ${ }^{8}$ F. L. Alves, ${ }^{9}$ G. A. Alves, ${ }^{9}$ M. Correa Martins Junior, ${ }^{9}$ G. Correia Silva, ${ }^{9}$ C. Hensel, ${ }^{9}$ A. Moraes, ${ }^{9}$ M. E. Pol, ${ }^{9}$ P. Rebello Teles, ${ }^{9}$ E. Belchior Batista Das Chagas, ${ }^{10}$ W. Carvalho, ${ }^{10}$ J. Chinellato, ${ }^{10, d}$ E. Coelho, ${ }^{10}$ E. M. Da Costa, ${ }^{10}$ G. G. Da Silveira, ${ }^{10, e}$ D. De Jesus Damiao, ${ }^{10}$ C. De Oliveira Martins,${ }^{10}$ S. Fonseca De Souza ${ }^{10}$ H. Malbouisson, ${ }^{10}$ D. Matos Figueiredo, ${ }^{10}$ M. Melo De Almeida, ${ }^{10}$ C. Mora Herrera, ${ }^{10}$ L. Mundim, ${ }^{10}$ H. Nogima, ${ }^{10}$ W. L. Prado Da Silva, ${ }^{10}$ L. J. Sanchez Rosas, ${ }^{10}$ A. Santoro, ${ }^{10}$ A. Sznajder, ${ }^{10}$ M. Thiel,${ }^{10}$ E. J. Tonelli Manganote,${ }^{10, d}$ F. Torres Da Silva De Araujo, ${ }^{10}$ A. Vilela Pereira, ${ }^{10}$ S. Ahuja, ${ }^{11 \mathrm{a}}$ C. A. Bernardes, ${ }^{11 \mathrm{a}}$ L. Calligaris, ${ }^{11 \mathrm{a}}$ T. R. Fernandez Perez Tomei, ${ }^{11 \mathrm{a}}$ E. M. Gregores,${ }^{11 \mathrm{a}, 1 \mathrm{~b}}$ P. G. Mercadante, ${ }^{11 a, 11 b}$ S. F. Novaes, ${ }^{11 a}$ Sandra S. Padula, ${ }^{11 a}$ A. Aleksandrov, ${ }^{12}$ R. Hadjiiska, ${ }^{12}$ P. Iaydjiev, ${ }^{12}$ A. Marinov, ${ }^{12}$ M. Misheva, ${ }^{12}$ M. Rodozov, ${ }^{12}$ M. Shopova, ${ }^{12}$ G. Sultanov, ${ }^{12}$ A. Dimitrov, ${ }^{13}$ L. Litov, ${ }^{13}$ B. Pavlov, ${ }^{13}$ P. Petkov, ${ }^{13}$ W. Fang,,${ }^{14, f}$ X. Gao, ${ }^{14, \mathrm{f}}$ L. Yuan, ${ }^{14}$ M. Ahmad, ${ }^{15}$ J. G. Bian, ${ }^{15}$ G. M. Chen, ${ }^{15}$ H. S. Chen, ${ }^{15}$ M. Chen, ${ }^{15}$ Y. Chen, ${ }^{15}$ C. H. Jiang, ${ }^{15}$ D. Leggat, ${ }^{15}$ H. Liao, ${ }^{15}$ Z. Liu, ${ }^{15}$ F. Romeo, ${ }^{15}$ S. M. Shaheen, ${ }^{15, g}$ A. Spiezia, ${ }^{15}$ J. Tao, ${ }^{15}$ Z. Wang, ${ }^{15}$ E. Yazgan, ${ }^{15}$ H. Zhang, ${ }^{15}$ S. Zhang, ${ }^{15, \mathrm{~g}}$ J. Zhao, ${ }^{15}$ Y. Ban, ${ }^{16}$ G. Chen,${ }^{16}$ A. Levin, ${ }^{16} \mathrm{~J} . \mathrm{Li},{ }^{16} \mathrm{~L} . \mathrm{Li},{ }^{16} \mathrm{Q} . \mathrm{Li},{ }^{16}$ Y. Mao, ${ }^{16} \mathrm{~S}$. J. Qian, ${ }^{16} \mathrm{D}$. Wang, ${ }^{16} \mathrm{Z} . \mathrm{Xu},{ }^{16}$ Y. Wang, ${ }^{17}$ C. Avila, ${ }^{18}$ A. Cabrera, ${ }^{18}$ C. A. Carrillo Montoya, ${ }^{18}$ L. F. Chaparro Sierra, ${ }^{18}$ C. Florez, ${ }^{18}$

C. F. González Hernández, ${ }^{18}$ M. A. Segura Delgado, ${ }^{18}$ B. Courbon, ${ }^{19}$ N. Godinovic, ${ }^{19}$ D. Lelas, ${ }^{19}$ I. Puljak, ${ }^{19}$ T. Sculac, ${ }^{19}$ Z. Antunovic, ${ }^{20}$ M. Kovac, ${ }^{20}$ V. Brigljevic, ${ }^{21}$ D. Ferencek, ${ }^{21}$ K. Kadija, ${ }^{21}$ B. Mesic, ${ }^{21}$ A. Starodumov, ${ }^{21, h}$ T. Susa, ${ }^{21}$ M. W. Ather, ${ }^{22}$ A. Attikis, ${ }^{22}$ M. Kolosova, ${ }^{22}$ G. Mavromanolakis,, 2 J. Mousa, ${ }^{22}$ C. Nicolaou, ${ }^{22}$ F. Ptochos, ${ }^{22}$ P. A. Razis, ${ }^{22}$ H. Rykaczewski, ${ }^{22}$ M. Finger, ${ }^{23, i}$ M. Finger Jr., ${ }^{23, i}$ E. Ayala, ${ }^{24}$ E. Carrera Jarrin, ${ }^{25}$ H. Abdalla, ${ }^{26, j}$ A. A. Abdelalim, ${ }^{26, k, 1}$ M. A. Mahmoud ${ }^{26, \mathrm{~m}, \mathrm{n}}$ S. Bhowmik, ${ }^{27}$ A. Carvalho Antunes De Oliveira, ${ }^{27}$ R. K. Dewanjee, ${ }^{27}$ K. Ehataht, ${ }^{27}$ M. Kadastik, ${ }^{27}$ M. Raidal, ${ }^{27}$ C. Veelken, ${ }^{27}$ P. Eerola,${ }^{28}$ H. Kirschenmann, ${ }^{28}$ J. Pekkanen, ${ }^{28}$ M. Voutilainen, ${ }^{28}$ J. Havukainen, ${ }^{29}$ J. K. Heikkilä, ${ }^{29}$ T. Järvinen, ${ }^{29}$ V. Karimäki, ${ }^{29}$ R. Kinnunen, ${ }^{29}$ T. Lampén, ${ }^{29}$ K. Lassila-Perini, ${ }^{29}$ S. Laurila, ${ }^{29}$ S. Lehti, ${ }^{29}$ T. Lindén, ${ }^{29}$ P. Luukka, ${ }^{29}$ T. Mäenpää, ${ }^{29}$ H. Siikonen, ${ }^{29}$ E. Tuominen, ${ }^{29}$ J. Tuominiemi, ${ }^{29}$ T. Tuuva, ${ }^{30}$ M. Besancon, ${ }^{31}$

F. Couderc, ${ }^{31}$ M. Dejardin, ${ }^{31}$ D. Denegri, ${ }^{31}$ J. L. Faure, ${ }^{31}$ F. Ferri, ${ }^{31}$ S. Ganjour, ${ }^{31}$ A. Givernaud, ${ }^{31}$ P. Gras, ${ }^{31}$ G. Hamel de Monchenault, ${ }^{31}$ P. Jarry, ${ }^{31}$ C. Leloup,${ }^{31}$ E. Locci, ${ }^{31}$ J. Malcles,${ }^{31}$ G. Negro, ${ }^{31}$ J. Rander, ${ }^{31}$ A. Rosowsky, ${ }^{31}$ M. Ö. Sahin, ${ }^{31}$ M. Titov, ${ }^{31}$ A. Abdulsalam, ${ }^{32,0}$ C. Amendola,${ }^{32}$ I. Antropov, ${ }^{32}$ F. Beaudette, ${ }^{32}$ P. Busson, ${ }^{32}$ C. Charlot, ${ }^{32}$ R. Granier de Cassagnac, ${ }^{32}$ I. Kucher, ${ }^{32}$ A. Lobanov, ${ }^{32}$ J. Martin Blanco, ${ }^{32}$ C. Martin Perez, ${ }^{32}$ M. Nguyen, ${ }^{32}$ C. Ochando, ${ }^{32}$ G. Ortona, ${ }^{32}$ P. Paganini ${ }^{32}$ P. Pigard ${ }^{32}$ J. Rembser, ${ }^{32}$ R. Salerno, ${ }^{32}$ J. B. Sauvan, ${ }^{32}$ Y. Sirois, ${ }^{32}$ A. G. Stahl Leiton, ${ }^{32}$ A. Zabi, ${ }^{32}$ A. Zghiche, ${ }^{32}$ J.-L. Agram, ${ }^{33, p}$ J. Andrea, ${ }^{33}$ D. Bloch,${ }^{33}$ J.-M. Brom, ${ }^{33}$ E. C. Chabert, ${ }^{33}$ V. Cherepanov, ${ }^{33}$ C. Collard, ${ }^{33}$ E. Conte, ${ }^{33, p}$ J.-C. Fontaine, ${ }^{33, p}$ D. Gelé, ${ }^{33}$ U. Goerlach, ${ }^{33}$ M. Jansová, ${ }^{33}$ A.-C. Le Bihan, ${ }^{33}$ N. Tonon, ${ }^{33}$ P. Van Hove, ${ }^{33}$ S. Gadrat,${ }^{34}$ S. Beauceron,${ }^{35}$ C. Bernet,${ }^{35}$ G. Boudoul, ${ }^{35}$ N. Chanon, ${ }^{35}$ R. Chierici, ${ }^{35}$ D. Contardo, ${ }^{35}$ P. Depasse,${ }^{35}$ H. El Mamouni, ${ }^{35}$ J. Fay, ${ }^{35}$ L. Finco, ${ }^{35}$ S. Gascon, ${ }^{35}$ M. Gouzevitch, ${ }^{35}$ G. Grenier, ${ }^{35}$ B. Ille,${ }^{35}$ F. Lagarde,${ }^{35}$ I. B. Laktineh, ${ }^{35}$ H. Lattaud,${ }^{35}$ M. Lethuillier, ${ }^{35}$ L. Mirabito, ${ }^{35}$ S. Perries, ${ }^{35}$ A. Popov, ${ }^{35, q}$ V. Sordini, ${ }^{35}$ G. Touquet, ${ }^{35}$ M. Vander Donckt, ${ }^{35}$ S. Viret, ${ }^{35}$ T. Toriashvili, ${ }^{36, \mathrm{r}}$ Z. Tsamalaidze, ${ }^{37, \mathrm{i}}$ C. Autermann, ${ }^{38}$ L. Feld, ${ }^{38} \mathrm{M}$. K. Kiesel, ${ }^{38}$ K. Klein,${ }^{38}$ M. Lipinski, ${ }^{38}$ M. Preuten, ${ }^{38}$ M. P. Rauch, ${ }^{38}$ C. Schomakers, ${ }^{38}$ J. Schulz,${ }^{38}$ M. Teroerde,${ }^{38}$ B. Wittmer, ${ }^{38}$ A. Albert, ${ }^{39}$ D. Duchardt, ${ }^{39}$ M. Erdmann, ${ }^{39}$ S. Erdweg, ${ }^{39}$ T. Esch,${ }^{39}$ R. Fischer, ${ }^{39}$ S. Ghosh,${ }^{39}$ A. Güth,${ }^{39}$ T. Hebbeker, ${ }^{39}$ C. Heidemann, ${ }^{39}$ K. Hoepfner, ${ }^{39}$ H. Keller, ${ }^{39}$ L. Mastrolorenzo, ${ }^{39}$ M. Merschmeyer, ${ }^{39}$ A. Meyer, ${ }^{39}$ P. Millet, ${ }^{39}$ S. Mukherjee, ${ }^{39}$ T. Pook, ${ }^{39}$ M. Radziej, ${ }^{39}$ H. Reithler, ${ }^{39}$ M. Rieger, ${ }^{39}$ A. Schmidt, ${ }^{39}$ D. Teyssier, ${ }^{39}$ S. Thüer, ${ }^{39}$ G. Flügge, ${ }^{40}$ O. Hlushchenko, ${ }^{40}$ T. Kress, ${ }^{40}$

A. Künsken, ${ }^{40}$ T. Müller, ${ }^{40}$ A. Nehrkorn, ${ }^{40}$ A. Nowack,${ }^{40}$ C. Pistone, ${ }^{40}$ O. Pooth,${ }^{40}$ D. Roy, ${ }^{40}$ H. Sert, ${ }^{40}$ A. Stahl, ${ }^{40, s}$ M. Aldaya Martin, ${ }^{41}$ T. Arndt, ${ }^{41}$ C. Asawatangtrakuldee, ${ }^{41}$ I. Babounikau, ${ }^{41}$ K. Beernaert, ${ }^{41}$ O. Behnke, ${ }^{41}$ U. Behrens, ${ }^{41}$ A. Bermúdez Martínez, ${ }^{41}$ D. Bertsche, ${ }^{41}$ A. A. Bin Anuar, ${ }^{41}$ K. Borras, ${ }^{41, t}$ V. Botta ${ }^{41}$ A. Campbell, ${ }^{41}$ P. Connor, ${ }^{41}$ C. Contreras-Campana, ${ }^{41}$ V. Danilov, ${ }^{41}$ A. De Wit,${ }^{41}$ M. M. Defranchis, ${ }^{41}$ C. Diez Pardos, ${ }^{41}$ D. Domínguez Damiani, ${ }^{41}$ G. Eckerlin, ${ }^{41}$ T. Eichhorn, ${ }^{41}$ A. Elwood, ${ }^{41}$ E. Eren, ${ }^{41}$ E. Gallo, ${ }^{41, u}$ A. Geiser, ${ }^{41}$ J. M. Grados Luyando, ${ }^{41}$ A. Grohsjean, ${ }^{41}$ M. Guthoff, ${ }^{41}$ M. Haranko, ${ }^{41}$ A. Harb, ${ }^{41}$ J. Hauk, ${ }^{41}$ H. Jung, ${ }^{41}$ M. Kasemann,${ }^{41}$ J. Keaveney, ${ }^{41}$ C. Kleinwort, ${ }^{41}$ J. Knolle, ${ }^{41}$ D. Krücker, ${ }^{41}$ W. Lange, ${ }^{41}$ A. Lelek, ${ }^{41}$ T. Lenz, ${ }^{41}$ J. Leonard, ${ }^{41}$ K. Lipka, ${ }^{41}$ W. Lohmann, ${ }^{41, v}$ R. Mankel, ${ }^{41}$ I.-A. Melzer-Pellmann, ${ }^{41}$ A. B. Meyer ${ }^{41}$ M. Meyer ${ }^{41}$ M. Missiroli, ${ }^{41}$ G. Mittag, ${ }^{41}$ J. Mnich, ${ }^{41}$ V. Myronenko, ${ }^{41}$ S. K. Pflitsch, ${ }^{41}$ D. Pitzl,${ }^{41}$ A. Raspereza, ${ }^{41}$ M. Savitskyi,${ }^{41}$ P. Saxena, ${ }^{41}$ P. Schütze, ${ }^{41}$ C. Schwanenberger, ${ }^{41}$ R. Shevchenko, ${ }^{41}$ A. Singh, ${ }^{41}$ H. Tholen, ${ }^{41}$ O. Turkot ${ }^{41}$ A. Vagnerini, ${ }^{41}$ G. P. Van Onsem, ${ }^{41}$ R. Walsh, ${ }^{41}$ Y. Wen, ${ }^{41}$ K. Wichmann, ${ }^{41}$ C. Wissing, ${ }^{41}$ O. Zenaiev, ${ }^{41}$ R. Aggleton, ${ }^{42}$ S. Bein, ${ }^{42}$ L. Benato ${ }^{42}$ A. Benecke, ${ }^{42}$ V. Blobel, ${ }^{42}$ T. Dreyer, ${ }^{42}$ A. Ebrahimi, ${ }^{42}$ E. Garutti, ${ }^{42}$ D. Gonzalez, ${ }^{42}$ P. Gunnellini, ${ }^{42}$ J. Haller, ${ }^{42}$ A. Hinzmann, ${ }^{42}$ A. Karavdina, ${ }^{42}$ G. Kasieczka, ${ }^{42}$ 
R. Klanner, ${ }^{42}$ R. Kogler, ${ }^{42}$ N. Kovalchuk, ${ }^{42}$ S. Kurz,${ }^{42}$ V. Kutzner, ${ }^{42}$ J. Lange, ${ }^{42}$ D. Marconi, ${ }^{42}$ J. Multhaup,${ }^{42}$ M. Niedziela, ${ }^{42}$ C. E. N. Niemeyer, ${ }^{42}$ D. Nowatschin, ${ }^{42}$ A. Perieanu, ${ }^{42}$ A. Reimers, ${ }^{42}$ O. Rieger, ${ }^{42}$ C. Scharf,,${ }^{42}$ P. Schleper, ${ }^{42}$ S. Schumann, ${ }^{42}$ J. Schwandt, ${ }^{42}$ J. Sonneveld, ${ }^{42}$ H. Stadie, ${ }^{42}$ G. Steinbrück, ${ }^{42}$ F. M. Stober, ${ }^{42}$ M. Stöver, ${ }^{42}$ A. Vanhoefer, ${ }^{42}$ B. Vormwald ${ }^{42}$ I. Zoi, ${ }^{42}$ M. Akbiyik, ${ }^{43}$ C. Barth, ${ }^{43}$ M. Baselga, ${ }^{43}$ S. Baur, ${ }^{43}$ E. Butz, ${ }^{43}$ R. Caspart, ${ }^{43}$ T. Chwalek, ${ }^{43}$ F. Colombo, ${ }^{43}$ W. De Boer ${ }^{43}$ A. Dierlamm, ${ }^{43}$ K. El Morabit ${ }^{43}$ N. Faltermann, ${ }^{43}$ B. Freund, ${ }^{43}$ M. Giffels, ${ }^{43}$ M. A. Harrendorf, ${ }^{43}$ F. Hartmann, ${ }^{43,5}$ S. M. Heindl, ${ }^{43}$ U. Husemann, ${ }^{43}$ I. Katkov, ${ }^{43, q}$ S. Kudella, ${ }^{43}$ S. Mitra, ${ }^{43}$ M. U. Mozer, ${ }^{43}$ Th. Müller, ${ }^{43}$ M. Musich, ${ }^{43}$ M. Plagge, ${ }^{43}$ G. Quast ${ }^{43}$ K. Rabbertz, ${ }^{43}$ M. Schröder, ${ }^{43}$ I. Shvetsov, ${ }^{43}$ H. J. Simonis, ${ }^{43}$ R. Ulrich, ${ }^{43}$ S. Wayand, ${ }^{43}$ M. Weber, ${ }^{43}$ T. Weiler, ${ }^{43}$ C. Wöhrmann, ${ }^{43}$ R. Wolf, ${ }^{43}$ G. Anagnostou, ${ }^{44}$ G. Daskalakis, ${ }^{44}$ T. Geralis, ${ }^{44}$ A. Kyriakis, ${ }^{44}$ D. Loukas, ${ }^{44}$ G. Paspalaki, ${ }^{44}$ I. Topsis-Giotis ${ }^{44}$ G. Karathanasis, ${ }^{45}$ S. Kesisoglou, ${ }^{45}$ P. Kontaxakis ${ }^{45}$ A. Panagiotou, ${ }^{45}$ I. Papavergou, ${ }^{45}$ N. Saoulidou, ${ }^{45}$ E. Tziaferi, ${ }^{45}$ K. Vellidis, ${ }^{45}$ K. Kousouris,${ }^{46}$ I. Papakrivopoulos, ${ }^{46}$ G. Tsipolitis, ${ }^{46}$ I. Evangelou, ${ }^{47}$ C. Foudas,${ }^{47}$ P. Gianneios, ${ }^{47}$ P. Katsoulis,${ }^{47}$ P. Kokkas, ${ }^{47}$ S. Mallios, ${ }^{47}$ N. Manthos, ${ }^{47}$ I. Papadopoulos, ${ }^{47}$ E. Paradas, ${ }^{47}$ J. Strologas, ${ }^{47}$ F. A. Triantis, ${ }^{47}$ D. Tsitsonis,${ }^{47}$ M. Bartók,${ }^{48, w}$ M. Csanad,${ }^{48}$ N. Filipovic, ${ }^{48}$ P. Major, ${ }^{48}$ M. I. Nagy, ${ }^{48}$ G. Pasztor, ${ }^{48}$ O. Surányi,${ }^{48}$ G. I. Veres,${ }^{48}$ G. Bencze,${ }^{49}$ C. Hajdu, ${ }^{49}$ D. Horvath, ${ }^{49, x}$ Á. Hunyadi, ${ }^{49}$ F. Sikler, ${ }^{49}$ T. Á. Vámi, ${ }^{49}$ V. Veszpremi, ${ }^{49}$ G. Vesztergombi, ${ }^{49, a, w}$ N. Beni,${ }^{50}$ S. Czellar, ${ }^{50}$ J. Karancsi, ${ }^{50, y}$ A. Makovec, ${ }^{50}$ J. Molnar, ${ }^{50}$ Z. Szillasi, ${ }^{50}$ P. Raics, ${ }^{51}$ Z. L. Trocsanyi,${ }^{51}$ B. Ujvari, ${ }^{51}$ S. Choudhury, ${ }^{52}$ J. R. Komaragiri, ${ }^{52}$ P. C. Tiwari, ${ }^{52}$ S. Bahinipati, ${ }^{53, z}$ C. Kar, ${ }^{53}$ P. Mal, ${ }^{53}$ K. Mandal, ${ }^{53}$ A. Nayak, ${ }^{53, a a}$ D. K. Sahoo,${ }^{53, z}$ S. K. Swain, ${ }^{53}$ S. Bansal,${ }^{54}$ S. B. Beri, ${ }^{54}$ V. Bhatnagar, ${ }^{54}$ S. Chauhan,${ }^{54}$ R. Chawla,${ }^{54}$ N. Dhingra, ${ }^{54}$ R. Gupta, ${ }^{54}$ A. Kaur,${ }^{54}$ M. Kaur, ${ }^{54}$ S. Kaur, ${ }^{54}$ P. Kumari, ${ }^{54}$ M. Lohan, ${ }^{54}$ A. Mehta,${ }^{54}$ K. Sandeep,${ }^{54}$ S. Sharma,${ }^{54}$ J. B. Singh,${ }^{54}$ A. K. Virdi, ${ }^{54}$ G. Walia, ${ }^{54}$ A. Bhardwaj,${ }^{55}$ B. C. Choudhary, ${ }^{55}$ R. B. Garg, ${ }^{55}$ M. Gola,${ }^{55}$ S. Keshri, ${ }^{55}$ Ashok Kumar, ${ }^{55}$ S. Malhotra, ${ }^{55}$ M. Naimuddin, ${ }^{55}$ P. Priyanka, ${ }^{55}$ K. Ranjan, ${ }^{55}$ Aashaq Shah, ${ }^{55}$ R. Sharma, ${ }^{55}$ R. Bhardwaj,${ }^{56, b b}$ M. Bharti,${ }^{56, b b}$ R. Bhattacharya,${ }^{56}$ S. Bhattacharya, ${ }^{56}$ U. Bhawandeep, ${ }^{56, b b}$ D. Bhowmik, ${ }^{56}$ S. Dey, ${ }^{56}$ S. Dutt, ${ }^{56, b b}$ S. Dutta, ${ }^{56}$ S. Ghosh,${ }^{56}$ K. Mondal, ${ }^{56}$ S. Nandan, ${ }^{56}$ A. Purohit, ${ }^{56}$

P. K. Rout, ${ }^{56}$ A. Roy, ${ }^{56}$ S. Roy Chowdhury, ${ }^{56}$ G. Saha, ${ }^{56}$ S. Sarkar,${ }^{56}$ M. Sharan, ${ }^{56}$ B. Singh, ${ }^{56, b b}$ S. Thakur, ${ }^{56, b b}$ P. K. Behera, ${ }^{57}$ R. Chudasama,${ }^{58}$ D. Dutta,${ }^{58}$ V. Jha,${ }^{58}$ V. Kumar, ${ }^{58}$ P. K. Netrakanti, ${ }^{58}$ L. M. Pant,${ }^{58}$ P. Shukla,${ }^{58}$ T. Aziz,${ }^{59}$ M. A. Bhat, ${ }^{59}$ S. Dugad, ${ }^{59}$ G. B. Mohanty,${ }^{59}$ N. Sur, ${ }^{59}$ B. Sutar ${ }^{59}$ Ravindra Kumar Verma, ${ }^{59}$ S. Banerjee, ${ }^{60}$ S. Bhattacharya,${ }^{60}$ S. Chatterjee, ${ }^{60}$ P. Das, ${ }^{60}$ M. Guchait, ${ }^{60}$ Sa. Jain, ${ }^{60}$ S. Karmakar, ${ }^{60}$ S. Kumar, ${ }^{60}$ M. Maity, ${ }^{60, c c}$ G. Majumder, ${ }^{60}$ K. Mazumdar, ${ }^{60}$ N. Sahoo, ${ }^{60}$ T. Sarkar, ${ }^{60, c c}$ S. Chauhan, ${ }^{61}$ S. Dube, ${ }^{61}$ V. Hegde,${ }^{61}$ A. Kapoor, ${ }^{61}$ K. Kothekar, ${ }^{61}$ S. Pandey, ${ }^{61}$ A. Rane, ${ }^{61}$ S. Sharma ${ }^{61}$ S. Chenarani, ${ }^{62, d d}$ E. Eskandari Tadavani, ${ }^{62}$ S. M. Etesami, ${ }^{62, d d}$ M. Khakzad, ${ }^{62}$

M. Mohammadi Najafabadi, ${ }^{62}$ M. Naseri, ${ }^{62}$ F. Rezaei Hosseinabadi, ${ }^{62}$ B. Safarzadeh,${ }^{62, e e}$ M. Zeinali, ${ }^{62}$ M. Felcini, ${ }^{63}$ M. Grunewald, ${ }^{63}$ M. Abbrescia, ${ }^{64 a, 64 b}$ C. Calabria,${ }^{64 a, 64 b}$ A. Colaleo, ${ }^{64 a}$ D. Creanza, ${ }^{64 a, 64 c}$ L. Cristella, ${ }^{64 a, 64 b}$ N. De Filippis, ${ }^{64,64 \mathrm{c}}$ M. De Palma, ${ }^{64 a, 64 b}$ A. Di Florio,${ }^{64 a, 64 b}$ F. Errico, ${ }^{64 a, 64 b}$ L. Fiore ${ }^{64 a}$ A. Gelmi, ${ }^{64 a, 64 b}$ G. Iaselli, ${ }^{64 a, 64 c}$ M. Ince, ${ }^{64 a, 64 b}$ S. Lezki, ${ }^{64 a, 64 b}$ G. Maggi, ${ }^{64 a, 64 c}$ M. Maggi,${ }^{64 a}$ G. Miniello, ${ }^{64 a, 64 b}$ S. My, ${ }^{64 a, 64 b}$ S. Nuzzo, ${ }^{64 a, 64 b}$ A. Pompili, ${ }^{64 a, 64 b}$ G. Pugliese, ${ }^{64 a, 64 c}$ R. Radogna,${ }^{64 a}$ A. Ranieri, ${ }^{64 a}$ G. Selvaggi, ${ }^{64 a, 64 b}$ A. Sharma, ${ }^{64 a}$ L. Silvestris, ${ }^{64 a}$ R. Venditti, ${ }^{64 a}$ P. Verwilligen, ${ }^{64 a}$ G. Zito, ${ }^{64 a}$ G. Abbiendi, ${ }^{65 a}$ C. Battilana, ${ }^{65 a, 65 b}$ D. Bonacorsi, ${ }^{65 a, 65 b}$ L. Borgonovi, ${ }^{65 a, 65 b}$ S. Braibant-Giacomelli, ${ }^{65 a, 65 b}$ R. Campanini, ${ }^{65 a, 65 b}$ P. Capiluppi, ${ }^{65 a, 65 b}$ A. Castro, ${ }^{65 a, 65 b}$ F. R. Cavallo, ${ }^{65 a}$ S. S. Chhibra, ${ }^{65 a, 65 b}$ C. Ciocca, ${ }^{65 a}$ G. Codispoti,${ }^{65 a, 65 b}$ M. Cuffiani, ${ }^{65 a, 65 b}$ G. M. Dallavalle, ${ }^{65 a}$ F. Fabbri, ${ }^{65 a}$ A. Fanfani, ${ }^{65 a, 65 b}$ E. Fontanesi, ${ }^{65 a}$ P. Giacomelli, ${ }^{65 a}$ C. Grandi, ${ }^{65 a}$ L. Guiducci, ${ }^{65 a, 65 b}$ F. Iemmi, ${ }^{65 a, 65 b}$ S. Lo Meo, ${ }^{65 a}$ S. Marcellini, ${ }^{65 a}$ G. Masetti, ${ }^{65 a}$ A. Montanari, ${ }^{65 a}$ F. L. Navarria, ${ }^{65 a, 65 b}$ A. Perrotta,${ }^{65 a}$ F. Primavera, ${ }^{65 a, 65 b, s}$ T. Rovelli ${ }^{65 a, 65 b}$ G. P. Siroli,${ }^{65 a, 65 b}$ N. Tosi, ${ }^{65 a}$ S. Albergo, ${ }^{66,66 \mathrm{~b}}$ A. Di Mattia, ${ }^{66 \mathrm{a}}$ R. Potenza, ${ }^{66 \mathrm{a}, 66 \mathrm{~b}}$ A. Tricomi, ${ }^{66 \mathrm{a}, 66 \mathrm{~b}}$ C. Tuve, ${ }^{66 \mathrm{a}, 66 \mathrm{~b}}$ G. Barbagli, ${ }^{67 \mathrm{a}}$ K. Chatterjee, ${ }^{67 \mathrm{a}, 67 \mathrm{~b}}$ V. Ciulli, ${ }^{67 a, 67 b}$ C. Civinini, ${ }^{67 a}$ R. D’Alessandro, ${ }^{67 a, 67 b}$ E. Focardi, ${ }^{67 a, 67 b}$ G. Latino, ${ }^{67 a}$ P. Lenzi, ${ }^{67 a, 67 b}$ M. Meschini, ${ }^{67 a}$ S. Paoletti, ${ }^{67 a}$ L. Russo,${ }^{67 a, f f}$ G. Sguazzoni, ${ }^{67 a}$ D. Strom, ${ }^{67 a}$ L. Viliani, ${ }^{67 a}$ L. Benussi, ${ }^{68}$ S. Bianco,${ }^{68}$ F. Fabbri, ${ }^{68}$ D. Piccolo ${ }^{68}$ F. Ferro, ${ }^{69 a}$ F. Ravera ${ }^{69 a, 69 b}$ E. Robutti, ${ }^{6 a}$ S. Tosi, ${ }^{69 a, 69 b}$ A. Benaglia, ${ }^{70 a}$ A. Beschi, ${ }^{70 a, 70 b}$ F. Brivio, ${ }^{70 a, 70 b}$ V. Ciriolo, ${ }^{70 a, 70 b, s}$ S. Di Guida, ${ }^{70 a, 70 b, s}$ M. E. Dinardo,${ }^{70 a, 70 b}$ S. Fiorendi, ${ }^{70 a, 70 b}$ S. Gennai, ${ }^{70 a}$ A. Ghezzi, ${ }^{70 a, 70 b}$ P. Govoni, ${ }^{70 a, 70 b}$ M. Malberti ${ }^{70 a, 70 b}$

S. Malvezzi, ${ }^{70 a}$ A. Massironi, ${ }^{70,70 b}$ D. Menasce,${ }^{70 a}$ F. Monti, ${ }^{70 a}$ L. Moroni,${ }^{70 a}$ M. Paganoni, ${ }^{70 a, 70 b}$ D. Pedrini, ${ }^{70 a}$ S. Ragazzi, ${ }^{70,70 b}$ T. Tabarelli de Fatis, ${ }^{70 a, 70 b}$ D. Zuolo, ${ }^{70 a, 70 b}$ S. Buontempo, ${ }^{71 a}$ N. Cavallo, ${ }^{71 a, 71 c}$ A. De Iorio, ${ }^{71 a, 71 b}$ A. Di Crescenzo, ${ }^{71 a, 71 b}$ F. Fabozzi, ${ }^{71 a, 71 c}$ F. Fienga, ${ }^{71 a}$ G. Galati, ${ }^{71 a}$ A. O. M. Iorio, ${ }^{71 a, 71 b}$ W. A. Khan, ${ }^{71 a}$ L. Lista, ${ }^{71 a}$ S. Meola, ${ }^{71 \mathrm{a}, 71 \mathrm{~d}, \mathrm{~s}}$ P. Paolucci, ${ }^{71 \mathrm{a}, \mathrm{s}}$ C. Sciacca, ${ }^{71 \mathrm{a}, 71 \mathrm{~b}}$ E. Voevodina, ${ }^{71 \mathrm{a}, 71 \mathrm{~b}}$ P. Azzi, ${ }^{72 \mathrm{a}}$ N. Bacchetta, ${ }^{72 \mathrm{a}}$ A. Boletti, ${ }^{72 \mathrm{a}, 72 \mathrm{~b}}$ A. Bragagnolo, ${ }^{72 a}$ R. Carlin, ${ }^{72 a, 72 b}$ P. Checchia ${ }^{72 a}$ M. Dall'Osso, ${ }^{72 a, 72 b}$ P. De Castro Manzano, ${ }^{72 a}$ T. Dorigo, ${ }^{72 a}$ U. Dosselli, ${ }^{72 a}$ F. Gasparini, ${ }^{72 a, 72 b}$ U. Gasparini, ${ }^{72 a, 72 b}$ S. Y. Hoh,${ }^{72 a}$ S. Lacaprara, ${ }^{72 a}$ P. Lujan, ${ }^{72 a}$ M. Margoni ${ }^{72 a, 72 b}$ A. T. Meneguzzo ${ }^{72 a, 72 b}$ F. Montecassiano, ${ }^{72 a}$ J. Pazzini, ${ }^{72 a, 72 b}$ N. Pozzobon, ${ }^{72 a, 72 b}$ P. Ronchese, ${ }^{72 a, 72 b}$ R. Rossin, ${ }^{72 a, 72 b}$ F. Simonetto, ${ }^{72 a, 72 b}$ A. Tiko, ${ }^{72 a}$ 
E. Torassa, ${ }^{72 a}$ M. Tosi, ${ }^{72 a, 72 b}$ M. Zanetti, ${ }^{72 a, 72 b}$ P. Zotto, ${ }^{72 a, 72 b}$ G. Zumerle, ${ }^{72 a, 72 b}$ A. Braghieri, ${ }^{73 a}$ A. Magnani, ${ }^{73 a}$ P. Montagna, ${ }^{73 a, 73 b}$ S. P. Ratti, ${ }^{73 a, 73 b}$ V. Re, ${ }^{73 a}$ M. Ressegotti, ${ }^{73 a, 73 b}$ C. Riccardi, ${ }^{73 a, 73 b}$ P. Salvini, ${ }^{73 a}$ I. Vai, $^{73 a, 73 b}$ P. Vitulo, ${ }^{73 a, 73 b}$ M. Biasini, ${ }^{74 a, 74 b}$ G. M. Bilei, ${ }^{74 a}$ C. Cecchi, ${ }^{74 a, 74 b}$ D. Ciangottini, ${ }^{74 a, 74 b}$ L. Fanò, ${ }^{74 a, 74 b}$ P. Lariccia, ${ }^{74 a, 74 b}$ R. Leonardi, ${ }^{74 a, 74 b}$ E. Manoni, ${ }^{74 a}$ G. Mantovani, ${ }^{74 a, 74 b}$ V. Mariani, ${ }^{74 a, 74 b}$ M. Menichelli, ${ }^{74 a}$ A. Rossi, ${ }^{74 a, 74 b}$ A. Santocchia, ${ }^{74,74 b}$ D. Spiga, ${ }^{74 a}$ K. Androsov, ${ }^{75 a}$ P. Azzurri, ${ }^{75 a}$ G. Bagliesi, ${ }^{75 a}$ L. Bianchini, ${ }^{75 a}$ T. Boccali, ${ }^{75 a}$ L. Borrello, ${ }^{75 a}$ R. Castaldi, ${ }^{75 a}$ M. A. Ciocci, ${ }^{75 a, 75 b}$ R. Dell'Orso, ${ }^{75 a}$ G. Fedi, ${ }^{75 a}$ F. Fiori, ${ }^{75 a, 75 c}$ L. Giannini, ${ }^{75 a, 75 c}$ A. Giassi, ${ }^{75 a}$ M. T. Grippo, ${ }^{75 a}$ F. Ligabue, ${ }^{75 a, 75 c}$ E. Manca, ${ }^{75 a, 75 c}$ G. Mandorli, ${ }^{75 a, 75 c}$ A. Messineo, ${ }^{75 a, 75 b}$ F. Palla, ${ }^{75 a}$ A. Rizzi, ${ }^{75 a, 75 b}$ G. Rolandi, ${ }^{75 a, g g}$ P. Spagnolo, ${ }^{75 a}$ R. Tenchini, ${ }^{75 a}$ G. Tonelli, ${ }^{75 a, 75 b}$ A. Venturi, ${ }^{75 a}$ P. G. Verdini, ${ }^{75 a}$ L. Barone, ${ }^{76 a, 76 b}$ F. Cavallari, ${ }^{76 a}$ M. Cipriani, ${ }^{76 a, 76 b}$ D. Del Re, ${ }^{76 a, 76 b}$ E. Di Marco, ${ }^{76 a, 76 b}$ M. Diemoz, ${ }^{76 a}$ S. Gelli, ${ }^{76 a, 76 b}$ E. Longo, ${ }^{76 a, 76 b}$ B. Marzocchi, ${ }^{76 a, 76 b}$ P. Meridiani, ${ }^{76 a}$ G. Organtini, ${ }^{76 a, 76 b}$ F. Pandolfi, ${ }^{76 a}$ R. Paramatti, ${ }^{76 a, 76 b}$ F. Preiato, ${ }^{76 a, 76 b}$ S. Rahatlou, ${ }^{76 a, 76 b}$ C. Rovelli, ${ }^{76 a}$ F. Santanastasio, ${ }^{76 a, 76 b}$ N. Amapane, ${ }^{77 a, 77 b}$ R. Arcidiacono, ${ }^{77 a, 77 c}$ S. Argiro, ${ }^{77 a, 77 b}$ M. Arneodo, ${ }^{77 a, 77 c}$ N. Bartosik, ${ }^{77 a}$ R. Bellan, ${ }^{77,77 b}$ C. Biino, ${ }^{77 a}$ N. Cartiglia, ${ }^{77 a}$ F. Cenna, ${ }^{77,77 b}$ S. Cometti, ${ }^{77 a}$ M. Costa, ${ }^{77 a, 77 b}$ R. Covarelli, ${ }^{77 a, 77 b}$ N. Demaria, ${ }^{77 a}$ B. Kiani, ${ }^{77 a, 77 b}$ C. Mariotti, ${ }^{77 a}$ S. Maselli, ${ }^{77 a}$ E. Migliore, ${ }^{77 a, 77 b}$ V. Monaco, ${ }^{77 a, 77 b}$ E. Monteil, ${ }^{77 a, 77 b}$ M. Monteno, ${ }^{77 a}$ M. M. Obertino, ${ }^{77 a, 77 b}$ L. Pacher, ${ }^{77,77 b}$ N. Pastrone, ${ }^{77 a}$

M. Pelliccioni, ${ }^{77 a}$ G. L. Pinna Angioni, ${ }^{77 a, 77 b}$ A. Romero, ${ }^{77 a, 77 b}$ M. Ruspa, ${ }^{77 a, 77 c}$ R. Sacchi, ${ }^{77 a, 77 b}$ K. Shchelina, ${ }^{77 a, 77 b}$ V. Sola, ${ }^{77 a}$ A. Solano, ${ }^{77 a, 77 b}$ D. Soldi, ${ }^{77 a, 77 b}$ A. Staiano, ${ }^{77 a}$ S. Belforte, ${ }^{78 a}$ V. Candelise ${ }^{78 a, 78 b}$ M. Casarsa, ${ }^{78 a}$ F. Cossutti, ${ }^{78 a}$ A. Da Rold, ${ }^{78 a, 78 b}$ G. Della Ricca, ${ }^{78 a, 78 b}$ F. Vazzoler, ${ }^{78 a, 78 b}$ A. Zanetti, ${ }^{78 a}$ D. H. Kim, ${ }^{79}$ G. N. Kim, ${ }^{79}$ M. S. Kim, ${ }^{79}$ J. Lee, ${ }^{79}$ S. Lee, ${ }^{79}$ S. W. Lee, ${ }^{79}$ C. S. Moon, ${ }^{79}$ Y. D. Oh, ${ }^{79}$ S. I. Pak, ${ }^{79}$ S. Sekmen, ${ }^{79}$ D. C. Son, ${ }^{79}$ Y. C. Yang, ${ }^{79}$ H. Kim, ${ }^{80}$ D. H. Moon, ${ }^{80}$ G. Oh, ${ }^{80}$ B. Francois, ${ }^{81}$ J. Goh, ${ }^{81, h h}$ T. J. Kim, ${ }^{81}$ S. Cho, ${ }^{82}$ S. Choi, ${ }^{82}$ Y. Go, ${ }^{82}$ D. Gyun, ${ }^{82}$ S. Ha, ${ }^{82}$ B. Hong, ${ }^{82}$ Y. Jo, ${ }^{82}$ K. Lee, ${ }^{82}$ K. S. Lee, ${ }^{82}$ S. Lee, ${ }^{82}$ J. Lim, ${ }^{82}$ S. K. Park, ${ }^{82}$ Y. Roh, ${ }^{82}$ H. S. Kim, ${ }^{83}$ J. Almond, ${ }^{84}$ J. Kim, ${ }^{84}$ J. S. Kim, ${ }^{84}$ H. Lee, ${ }^{84}$ K. Lee, ${ }^{84}$ K. Nam, ${ }^{84}$ S. B. Oh, ${ }^{84}$ B. C. Radburn-Smith, ${ }^{84}$ S. h. Seo, ${ }^{84}$ U. K. Yang, ${ }^{84}$ H. D. Yoo, ${ }^{84}$ G. B. Yu, ${ }^{84}$ D. Jeon, ${ }^{85}$ H. Kim, ${ }^{85}$ J. H. Kim, ${ }^{85}$ J. S. H. Lee, ${ }^{85}$ I. C. Park, ${ }^{85}$ Y. Choi, ${ }^{86}$ C. Hwang, ${ }^{86}$ J. Lee, ${ }^{86}$ I. Yu, ${ }^{86}$ V. Dudenas, ${ }^{87}$ A. Juodagalvis, ${ }^{87}$

J. Vaitkus, ${ }^{87}$ I. Ahmed, ${ }^{88}$ Z. A. Ibrahim, ${ }^{88}$ M. A. B. Md Ali, ${ }^{88, i i}$ F. Mohamad Idris, ${ }^{88, j j}$ W. A. T. Wan Abdullah, ${ }^{88}$ M. N. Yusli, ${ }^{88}$ Z. Zolkapli, ${ }^{88}$ J. F. Benitez, ${ }^{89}$ A. Castaneda Hernandez, ${ }^{89}$ J. A. Murillo Quijada, ${ }^{89}$ H. Castilla-Valdez, ${ }^{90}$ E. De La Cruz-Burelo, ${ }^{90}$ M. C. Duran-Osuna, ${ }^{90}$ I. Heredia-De La Cruz, ${ }^{90, k k}$ R. Lopez-Fernandez, ${ }^{90}$ J. Mejia Guisao, $^{90}$ R. I. Rabadan-Trejo, ${ }^{90}$ M. Ramirez-Garcia, ${ }^{90}$ G. Ramirez-Sanchez, ${ }^{90}$ R. Reyes-Almanza, ${ }^{90}$ A. Sanchez-Hernandez, ${ }^{90}$ S. Carrillo Moreno, ${ }^{91}$ C. Oropeza Barrera, ${ }^{91}$ F. Vazquez Valencia, ${ }^{91}$ J. Eysermans, ${ }^{92}$ I. Pedraza, ${ }^{92}$ H. A. Salazar Ibarguen, ${ }^{92}$ C. Uribe Estrada, ${ }^{92}$ A. Morelos Pineda, ${ }^{93}$ D. Krofcheck, ${ }^{94}$ S. Bheesette, ${ }^{95}$ P. H. Butler, ${ }^{95}$ A. Ahmad, ${ }^{96}$ M. Ahmad, ${ }^{96}$ M. I. Asghar, ${ }^{96}$ Q. Hassan, ${ }^{96}$ H. R. Hoorani, ${ }^{96}$ A. Saddique, ${ }^{96}$ M. A. Shah, ${ }^{96}$ M. Shoaib, ${ }^{96}$ M. Waqas, ${ }^{96}$ H. Bialkowska, ${ }^{97}$ M. Bluj, ${ }^{97}$ B. Boimska, ${ }^{97}$ T. Frueboes, ${ }^{97}$ M. Górski, ${ }^{97}$ M. Kazana, ${ }^{97}$ M. Szleper, ${ }^{97}$ P. Traczyk, ${ }^{97}$ P. Zalewski, ${ }^{97}$ K. Bunkowski, ${ }^{98}$ A. Byszuk, ${ }^{98,11}$ K. Doroba, ${ }^{98}$ A. Kalinowski, ${ }^{98}$ M. Konecki, ${ }^{98}$ J. Krolikowski, $^{98}$ M. Misiura, ${ }^{98}$ M. Olszewski, ${ }^{98}$ A. Pyskir, ${ }^{98}$ M. Walczak, ${ }^{98}$ M. Araujo, ${ }^{99}$ P. Bargassa, ${ }^{99}$ C. Beirão Da Cruz E Silva, ${ }^{99}$ A. Di Francesco, ${ }^{99}$ P. Faccioli, ${ }^{99}$ B. Galinhas, ${ }^{99}$ M. Gallinaro, ${ }^{99}$ J. Hollar, ${ }^{99}$ N. Leonardo, ${ }^{99}$ J. Seixas, ${ }^{99}$ G. Strong, ${ }^{99}$ O. Toldaiev, ${ }^{99}$ J. Varela, ${ }^{99}$ S. Afanasiev, ${ }^{100}$ P. Bunin, ${ }^{100}$ M. Gavrilenko, ${ }^{100}$ I. Golutvin, ${ }^{100}$ I. Gorbunov, ${ }^{100}$ A. Kamenev, ${ }^{100}$ V. Karjavine, ${ }^{100}$ A. Lanev, ${ }^{100}$ A. Malakhov, ${ }^{100}$ V. Matveev, ${ }^{100, m m, n n}$ P. Moisenz, ${ }^{100}$ V. Palichik, ${ }^{100}$ V. Perelygin, ${ }^{100}$ S. Shmatov, ${ }^{100}$ S. Shulha, ${ }^{100}$ N. Skatchkov, ${ }^{100}$ V. Smirnov, ${ }^{100}$ N. Voytishin, ${ }^{100}$ A. Zarubin, ${ }^{100}$ V. Golovtsov, ${ }^{101}$ Y. Ivanov, ${ }^{101}$ V. Kim, ${ }^{101,00}$ E. Kuznetsova, ${ }^{101, p p}$ P. Levchenko, ${ }^{101}$ V. Murzin, ${ }^{101}$ V. Oreshkin, ${ }^{101}$ I. Smirnov, ${ }^{101}$ D. Sosnov, ${ }^{101}$ V. Sulimov, ${ }^{101}$ L. Uvarov, ${ }^{101}$ S. Vavilov, ${ }^{101}$ A. Vorobyev, ${ }^{101}$ Yu. Andreev, ${ }^{102}$ A. Dermenev, ${ }^{102}$ S. Gninenko, ${ }^{102}$ N. Golubev, ${ }^{102}$ A. Karneyeu, ${ }^{102}$ M. Kirsanov, ${ }^{102}$ N. Krasnikov, ${ }^{102}$ A. Pashenkov, ${ }^{102}$ D. Tlisov, ${ }^{102}$ A. Toropin, ${ }^{102}$ V. Epshteyn, ${ }^{103}$ V. Gavrilov, ${ }^{103}$ N. Lychkovskaya, ${ }^{103}$ V. Popov, ${ }^{103}$ I. Pozdnyakov, ${ }^{103}$ G. Safronov, ${ }^{103}$ A. Spiridonov, ${ }^{103}$ A. Stepennov, ${ }^{103}$ V. Stolin, ${ }^{103}$ M. Toms, ${ }^{103}$ E. Vlasov, ${ }^{103}$ A. Zhokin, ${ }^{103}$ T. Aushev, ${ }^{104}$ M. Chadeeva, ${ }^{105, q q}$ P. Parygin, ${ }^{105}$ D. Philippov, ${ }^{105}$ S. Polikarpov, ${ }^{105, q q}$ E. Popova, ${ }^{105}$ V. Rusinov, ${ }^{105}$ V. Andreev, ${ }^{106}$ M. Azarkin, ${ }^{106}$ I. Dremin, ${ }^{106, n n}$ M. Kirakosyan, ${ }^{106}$ S. V. Rusakov, ${ }^{106}$ A. Terkulov, ${ }^{106}$ A. Baskakov, ${ }^{107}$ A. Belyaev, ${ }^{107}$ E. Boos, ${ }^{107}$ V. Bunichev, ${ }^{107}$ M. Dubinin, ${ }^{107, r r}$ L. Dudko, ${ }^{107}$ A. Ershov, ${ }^{107}$ V. Klyukhin, ${ }^{107}$ O. Kodolova, ${ }^{107}$ I. Lokhtin, ${ }^{107}$ I. Miagkov, ${ }^{107}$ S. Obraztsov, ${ }^{107}$ S. Petrushanko, ${ }^{107}$ V. Savrin, ${ }^{107}$ A. Snigirev, ${ }^{107}$ A. Barnyakov, ${ }^{108, s s}$ V. Blinov, ${ }^{108, s s}$ T. Dimova, ${ }^{108, s s}$ L. Kardapoltsev, ${ }^{108, s s}$ Y. Skovpen, ${ }^{108, s s}$ I. Azhgirey, ${ }^{109}$ I. Bayshev, ${ }^{109}$ S. Bitioukov, ${ }^{109}$ D. Elumakhov, ${ }^{109}$ A. Godizov, ${ }^{109}$ V. Kachanov, ${ }^{109}$ A. Kalinin, ${ }^{109}$ D. Konstantinov, ${ }^{109}$ P. Mandrik, ${ }^{109}$ V. Petrov, ${ }^{109}$ R. Ryutin, ${ }^{109}$ S. Slabospitskii, ${ }^{109}$ A. Sobol, ${ }^{109}$ S. Troshin, ${ }^{109}$ N. Tyurin, ${ }^{109}$ A. Uzunian, ${ }^{109}$ A. Volkov, ${ }^{109}$ A. Babaev, ${ }^{110}$ S. Baidali, ${ }^{110}$ V. Okhotnikov, ${ }^{110}$ P. Adzic, ${ }^{111, t t}$ P. Cirkovic, ${ }^{111}$ D. Devetak, ${ }^{111}$ M. Dordevic, ${ }^{111}$ J. Milosevic, ${ }^{111}$ J. Alcaraz Maestre, ${ }^{112}$ A. Álvarez Fernández, ${ }^{112}$ I. Bachiller, ${ }^{112}$ M. Barrio Luna, ${ }^{112}$

J. A. Brochero Cifuentes, ${ }^{112}$ M. Cerrada, ${ }^{112}$ N. Colino, ${ }^{112}$ B. De La Cruz, ${ }^{112}$ A. Delgado Peris, ${ }^{112}$ C. Fernandez Bedoya, ${ }^{112}$ 
J. P. Fernández Ramos, ${ }^{112}$ J. Flix, ${ }^{112}$ M. C. Fouz, ${ }^{112}$ O. Gonzalez Lopez, ${ }^{112}$ S. Goy Lopez, ${ }^{112}$ J. M. Hernandez, ${ }^{112}$ M. I. Josa, ${ }^{112}$ D. Moran, ${ }^{112}$ A. Pérez-Calero Yzquierdo, ${ }^{112}$ J. Puerta Pelayo, ${ }^{112}$ I. Redondo, ${ }^{112}$ L. Romero, ${ }^{112}$ M. S. Soares, ${ }^{112}$ A. Triossi, ${ }^{112}$ C. Albajar, ${ }^{113}$ J. F. de Trocóniz, ${ }^{113}$ J. Cuevas, ${ }^{114}$ C. Erice, ${ }^{114}$ J. Fernandez Menendez, ${ }^{114}$ S. Folgueras, ${ }^{114}$ I. Gonzalez Caballero, ${ }^{114}$ J. R. González Fernández, ${ }^{114}$ E. Palencia Cortezon, ${ }^{114}$ V. Rodríguez Bouza, ${ }^{114}$ S. Sanchez Cruz, ${ }^{114}$ P. Vischia, ${ }^{114}$ J. M. Vizan Garcia, ${ }^{114}$ I. J. Cabrillo, ${ }^{115}$ A. Calderon, ${ }^{115}$ B. Chazin Quero, ${ }^{115}$ J. Duarte Campderros, ${ }^{115}$ M. Fernandez, ${ }^{115}$ P. J. Fernández Manteca, ${ }^{115}$ A. García Alonso, ${ }^{115}$ J. Garcia-Ferrero, ${ }^{115}$ G. Gomez, ${ }^{115}$ A. Lopez Virto, ${ }^{115}$ J. Marco, ${ }^{115}$ C. Martinez Rivero, ${ }^{115}$ P. Martinez Ruiz del Arbol, ${ }^{115}$ F. Matorras, ${ }^{115}$ J. Piedra Gomez, ${ }^{115}$ C. Prieels, ${ }^{115}$ T. Rodrigo, ${ }^{115}$ A. Ruiz-Jimeno, ${ }^{115}$ L. Scodellaro, ${ }^{115}$ N. Trevisani, ${ }^{115}$ I. Vila, ${ }^{115}$ R. Vilar Cortabitarte, ${ }^{115}$ N. Wickramage, ${ }^{116}$ D. Abbaneo ${ }^{117}$ B. Akgun, ${ }^{117}$ E. Auffray, ${ }^{117}$ G. Auzinger, ${ }^{117}$ P. Baillon, ${ }^{117}$ A. H. Ball, ${ }^{117}$ D. Barney, ${ }^{117}$ J. Bendavid, ${ }^{117}$ M. Bianco ${ }^{117}$ A. Bocci, ${ }^{117}$ C. Botta, ${ }^{117}$ E. Brondolin, ${ }^{117}$ T. Camporesi, ${ }^{117}$ M. Cepeda, ${ }^{117}$ G. Cerminara,${ }^{117}$ E. Chapon, ${ }^{117}$ Y. Chen, ${ }^{117}$ G. Cucciati, ${ }^{117}$ D. d'Enterria, ${ }^{117}$ A. Dabrowski, ${ }^{117}$ N. Daci, ${ }^{117}$ V. Daponte, ${ }^{117}$ A. David, ${ }^{117}$ A. De Roeck, ${ }^{117}$

N. Deelen, ${ }^{117}$ M. Dobson, ${ }^{117}$ M. Dünser, ${ }^{117}$ N. Dupont, ${ }^{117}$ A. Elliott-Peisert, ${ }^{117}$ P. Everaerts, ${ }^{117}$ F. Fallavollita, ${ }^{117, \text { uu }}$ D. Fasanella, ${ }^{117}$ G. Franzoni, ${ }^{117}$ J. Fulcher, ${ }^{117}$ W. Funk, ${ }^{117}$ D. Gigi, ${ }^{117}$ A. Gilbert, ${ }^{117}$ K. Gill, ${ }^{117}$ F. Glege,${ }^{117}$ M. Gruchala, ${ }^{117}$ M. Guilbaud, ${ }^{117}$ D. Gulhan, ${ }^{117}$ J. Hegeman, ${ }^{117}$ C. Heidegger, ${ }^{117}$ V. Innocente,${ }^{117}$ A. Jafari, ${ }^{117}$ P. Janot, ${ }^{117}$ O. Karacheban, ${ }^{117, v}$ J. Kieseler, ${ }^{117}$ A. Kornmayer ${ }^{117}$ M. Krammer, ${ }^{117, b}$ C. Lange, ${ }^{117}$ P. Lecoq,${ }^{117}$ C. Lourenço, ${ }^{117}$ L. Malgeri, ${ }^{117}$ M. Mannelli, ${ }^{117}$ F. Meijers, ${ }^{117}$ J. A. Merlin, ${ }^{117}$ S. Mersi, ${ }^{117}$ E. Meschi, ${ }^{117}$ P. Milenovic,${ }^{117, v v}$ F. Moortgat ${ }^{117}$ M. Mulders,${ }^{117}$ J. Ngadiuba, ${ }^{117}$ S. Nourbakhsh, ${ }^{117}$ S. Orfanelli, ${ }^{17}$ L. Orsini, ${ }^{177}$ F. Pantaleo, ${ }^{117, s}$ L. Pape, ${ }^{117}$ E. Perez, ${ }^{117}$ M. Peruzzi, ${ }^{117}$ A. Petrilli, ${ }^{117}$ G. Petrucciani, ${ }^{117}$ A. Pfeiffer, ${ }^{117}$ M. Pierini, ${ }^{117}$ F. M. Pitters, ${ }^{117}$ D. Rabady, ${ }^{117}$ A. Racz, ${ }^{117}$ T. Reis, ${ }^{117}$ M. Rovere, ${ }^{117}$ H. Sakulin, ${ }^{117}$ C. Schäfer, ${ }^{117}$ C. Schwick, ${ }^{117}$ M. Seidel, ${ }^{117}$ M. Selvaggi, ${ }^{117}$ A. Sharma, ${ }^{117}$ P. Silva, ${ }^{117}$ P. Sphicas, ${ }^{117}$,ww A. Stakia, ${ }^{117}$ J. Steggemann, ${ }^{117}$ D. Treille, ${ }^{117}$ A. Tsirou, ${ }^{117}$ V. Veckalns,,${ }^{17, x x}$ M. Verzetti, ${ }^{17}$ W. D. Zeuner, ${ }^{17}$

L. Caminada, ${ }^{118, y y}$ K. Deiters, ${ }^{118}$ W. Erdmann, ${ }^{118}$ R. Horisberger, ${ }^{118}$ Q. Ingram ${ }^{118}$ H. C. Kaestli, ${ }^{118}$ D. Kotlinski, ${ }^{118}$

U. Langenegger, ${ }^{118}$ T. Rohe, ${ }^{118}$ S. A. Wiederkehr, ${ }^{118}$ M. Backhaus, ${ }^{119}$ L. Bäni, ${ }^{119}$ P. Berger, ${ }^{119}$ N. Chernyavskaya, ${ }^{119}$ G. Dissertori, ${ }^{119}$ M. Dittmar, ${ }^{119}$ M. Donegà, ${ }^{119}$ C. Dorfer, ${ }^{119}$ T. A. Gómez Espinosa, ${ }^{119}$ C. Grab, ${ }^{119}$ D. Hits, ${ }^{119}$ T. Klijnsma, ${ }^{119}$ W. Lustermann, ${ }^{119}$ R. A. Manzoni, ${ }^{119}$ M. Marionneau, ${ }^{119}$ M. T. Meinhard, ${ }^{119}$ F. Micheli, ${ }^{119}$ P. Musella, ${ }^{119}$ F. Nessi-Tedaldi, ${ }^{119}$ J. Pata, ${ }^{119}$ F. Pauss, ${ }^{119}$ G. Perrin, ${ }^{119}$ L. Perrozzi, ${ }^{119}$ S. Pigazzini, ${ }^{119}$ M. Quittnat, ${ }^{119}$ C. Reissel, ${ }^{119}$ D. Ruini, ${ }^{119}$ D. A. Sanz Becerra, ${ }^{119}$ M. Schönenberger, ${ }^{119}$ L. Shchutska, ${ }^{119}$ V. R. Tavolaro ${ }_{119}{ }^{11}$. Theofilatos, ${ }^{119}$ M. L. Vesterbacka Olsson, ${ }^{119}$ R. Wallny, ${ }^{119}$ D. H. Zhu, ${ }^{119}$ T. K. Aarrestad, ${ }^{120}$ C. Amsler, ${ }^{120, z z}$ D. Brzhechko, ${ }^{120}$ M. F. Canelli, ${ }^{120}$ A. De Cosa, ${ }^{120}$ R. Del Burgo, ${ }^{120}$ S. Donato, ${ }^{120}$ C. Galloni,${ }^{120}$ T. Hreus, ${ }^{120}$ B. Kilminster, ${ }^{120}$ S. Leontsinis, ${ }^{120}$ I. Neutelings, ${ }^{120}$ G. Rauco, ${ }^{120}$ P. Robmann, ${ }^{120}$ K. Schweiger, ${ }^{120}$ C. Seitz ${ }^{120}$ Y. Takahashi, ${ }^{120}$ A. Zucchetta, ${ }^{120}$ Y. H. Chang, ${ }^{121}$ K. y. Cheng, ${ }^{121}$ T. H. Doan, ${ }^{121}$ R. Khurana, ${ }^{121}$ C. M. Kuo, ${ }^{121}$ W. Lin, ${ }^{121}$ A. Pozdnyakov, ${ }^{121}$ S. S. Yu, ${ }^{121}$ P. Chang, ${ }^{122}$ Y. Chao, ${ }^{122}$ K. F. Chen, ${ }^{122}$ P. H. Chen, ${ }^{122}$ W.-S. Hou, ${ }^{122}$ Arun Kumar, ${ }^{122}$ Y. F. Liu, ${ }^{122}$ R.-S. Lu, ${ }^{122}$ E. Paganis, ${ }^{122}$ A. Psallidas, ${ }^{122}$ A. Steen, ${ }^{122}$ B. Asavapibhop, ${ }^{123}$ N. Srimanobhas, ${ }^{123}$ N. Suwonjandee, ${ }^{123}$ A. Bat, ${ }^{124}$ F. Boran, ${ }^{124}$ S. Cerci, ${ }^{124, \text { aaa }}$ S. Damarseckin, ${ }^{124}$ Z. S. Demiroglu, ${ }^{124}$ F. Dolek, ${ }^{124}$ C. Dozen, ${ }^{124}$ I. Dumanoglu, ${ }^{124}$ S. Girgis, ${ }^{124}$ G. Gokbulut, ${ }^{124}$ Y. Guler, ${ }^{124}$ E. Gurpinar, ${ }^{124}$ I. Hos, ${ }^{124, \text { bb }}$ C. Isik, ${ }^{124}$ E. E. Kangal, ${ }^{124, c c c}$ O. Kara, ${ }^{124}$ A. Kayis Topaksu, ${ }^{124}$ U. Kiminsu, ${ }^{124}$ M. Oglakci, ${ }^{124}$ G. Onengut, ${ }^{124}$ K. Ozdemir, ${ }^{124, \text { ddd }}$ S. Ozturk, ${ }^{124, \text { eee }}$ D. Sunar Cerci, ${ }^{124, \text { aaa }}$ B. Tali, ${ }^{124, \text { aaa }}$ U. G. Tok, ${ }^{124}$ S. Turkcapar, ${ }^{124}$ I. S. Zorbakir, ${ }^{124}$ C. Zorbilmez, ${ }^{124}$ B. Isildak, ${ }^{125, f f f}$ G. Karapinar, ${ }^{125, g g g}$ M. Yalvac, ${ }^{125}$ M. Zeyrek, ${ }^{125}$ I. O. Atakisi, ${ }^{126}$ E. Gülmez, ${ }^{126}$ M. Kaya, ${ }^{126, \text { hhh }}$ O. Kaya, ${ }^{126, i i i}$ S. Ozkorucuklu, ${ }^{126, j j j}$ S. Tekten, ${ }^{126}$ E. A. Yetkin, ${ }^{126, k k k}$ M. N. Agaras, ${ }^{127}$ A. Cakir,${ }^{127}$ K. Cankocak, ${ }^{127}$ Y. Komurcu, ${ }^{127}$ S. Sen, ${ }^{127,111}$ B. Grynyov, ${ }^{128}$ L. Levchuk, ${ }^{129}$ F. Ball, ${ }^{130}$ L. Beck, ${ }^{130}$ J. J. Brooke, ${ }^{130}$ D. Burns, ${ }^{130}$ E. Clement, ${ }^{130}$ D. Cussans, ${ }^{130}$ O. Davignon, ${ }^{130}$ H. Flacher, ${ }^{130}$ J. Goldstein, ${ }^{130}$ G. P. Heath, ${ }^{130}$ H. F. Heath, ${ }^{130}$ L. Kreczko, ${ }^{130}$ D. M. Newbold, ${ }^{130, m m m}$ S. Paramesvaran, ${ }^{130}$ B. Penning, ${ }^{130}$ T. Sakuma, ${ }_{130}$ D. Smith ${ }^{130}$ V. J. Smith, ${ }^{130}$ J. Taylor, ${ }^{130}$ A. Titterton, ${ }^{130}$ K. W. Bell, ${ }^{131}$ A. Belyaev, ${ }^{131, n n n}$ C. Brew, ${ }^{131}$ R. M. Brown, ${ }^{131}$ D. Cieri, ${ }^{131}$ D. J. A. Cockerill, ${ }^{131}$ J. A. Coughlan, ${ }^{131}$ K. Harder, ${ }^{131}$ S. Harper, ${ }^{131}$ J. Linacre, ${ }^{131}$ E. Olaiya, ${ }^{131}$ D. Petyt, ${ }^{131}$ C. H. Shepherd-Themistocleous, ${ }^{131}$ A. Thea, ${ }^{131}$ I. R. Tomalin, ${ }^{131}$ T. Williams, ${ }^{131}$ W. J. Womersley, ${ }^{131}$ R. Bainbridge, ${ }^{132}$ P. Bloch, ${ }^{132}$ J. Borg ${ }^{132}$ S. Breeze ${ }^{132}$ O. Buchmuller, ${ }^{132}$ A. Bundock, ${ }^{132}$ D. Colling, ${ }^{132}$ P. Dauncey, ${ }^{132}$ G. Davies, ${ }^{132}$ M. Della Negra, ${ }^{132}$ R. Di Maria, ${ }^{132}$ G. Hall, ${ }^{132}$ G. Iles, ${ }^{132}$ T. James, ${ }^{132}$ M. Komm, ${ }^{132}$ C. Laner, ${ }^{132}$ L. Lyons, ${ }^{132}$ A.-M. Magnan, ${ }^{132}$ S. Malik, ${ }^{132}$ A. Martelli, ${ }^{132}$ J. Nash, ${ }^{132,000}$ A. Nikitenko, ${ }^{132, \mathrm{~h}}$ V. Palladino, ${ }^{132}$ M. Pesaresi, ${ }^{132}$ D. M. Raymond, ${ }^{132}$ A. Richards, ${ }^{132}$ A. Rose, ${ }^{132}$ E. Scott,,${ }^{132}$ C. Seez,${ }^{132}$ A. Shtipliyski, ${ }^{132}$ G. Singh, ${ }^{132}$ M. Stoye, ${ }^{132}$ T. Strebler, ${ }^{132}$ S. Summers, ${ }^{132}$ A. Tapper, ${ }^{132}$ K. Uchida, ${ }^{132}$ T. Virdee, ${ }^{132, s}$ N. Wardle, ${ }^{132}$ D. Winterbottom, ${ }^{132}$ J. Wright, ${ }^{132}$ S. C. Zenz, ${ }^{132}$ J. E. Cole, ${ }^{133}$ P. R. Hobson, ${ }^{133}$ A. Khan, ${ }^{133}$ P. Kyberd, ${ }^{133}$ C. K. Mackay, ${ }^{133}$ A. Morton, ${ }^{133}$ I. D. Reid, ${ }^{133}$ 
L. Teodorescu, ${ }^{133}$ S. Zahid, ${ }^{133}$ K. Call, ${ }^{134}$ J. Dittmann, ${ }^{134}$ K. Hatakeyama, ${ }^{134}$ H. Liu, ${ }^{134}$ C. Madrid, ${ }^{134}$ B. McMaster, ${ }^{134}$ N. Pastika, ${ }^{134}$ C. Smith, ${ }^{134}$ R. Bartek, ${ }^{135}$ A. Dominguez, ${ }^{135}$ A. Buccilli, ${ }^{136}$ S. I. Cooper, ${ }^{136}$ C. Henderson, ${ }^{136}$ P. Rumerio, ${ }^{136}$ C. West, ${ }^{136}$ D. Arcaro, ${ }^{137}$ T. Bose, ${ }^{137}$ D. Gastler, ${ }^{137}$ D. Pinna, ${ }^{137}$ D. Rankin, ${ }^{137}$ C. Richardson, ${ }^{137}$ J. Rohlf, ${ }^{137}$ L. Sulak, ${ }^{137}$ D. Zou, ${ }^{137}$ G. Benelli, ${ }^{138}$ X. Coubez, ${ }^{138}$ D. Cutts, ${ }^{138}$ M. Hadley, ${ }^{138}$ J. Hakala, ${ }^{138}$ U. Heintz, ${ }^{138}$ J. M. Hogan, ${ }^{138, p p p}$ K. H. M. Kwok, ${ }^{138}$ E. Laird, ${ }^{138}$ G. Landsberg, ${ }^{138}$ J. Lee, ${ }^{138}$ Z. Mao, ${ }^{138}$ M. Narain, ${ }^{138}$ S. Sagir, ${ }^{138, q q q}$ R. Syarif, ${ }^{138}$ E. Usai, ${ }^{138}$ D. Yu, ${ }^{138}$ R. Band, ${ }^{139}$ C. Brainerd, ${ }^{139}$ R. Breedon, ${ }^{139}$ D. Burns, ${ }^{139}$ M. Calderon De La Barca Sanchez, ${ }^{139}$ M. Chertok, ${ }^{139}$ J. Conway, ${ }^{139}$ R. Conway, ${ }^{139}$ P. T. Cox, ${ }^{139}$ R. Erbacher, ${ }^{139}$ C. Flores, ${ }^{139}$ G. Funk, ${ }^{139}$ W. Ko, ${ }^{139}$ O. Kukral, ${ }^{139}$ R. Lander, ${ }^{139}$ M. Mulhearn, ${ }^{139}$ D. Pellett, ${ }^{139}$ J. Pilot, ${ }^{139}$ S. Shalhout, ${ }^{139}$ M. Shi, ${ }^{139}$ D. Stolp, ${ }^{139}$ D. Taylor, ${ }^{139}$ K. Tos, ${ }^{139}$ M. Tripathi, ${ }^{139}$ Z. Wang, ${ }^{139}$ F. Zhang, ${ }^{139}$ M. Bachtis, ${ }^{140}$ C. Bravo, ${ }^{140}$ R. Cousins, ${ }^{140}$ A. Dasgupta, ${ }^{140}$ A. Florent, ${ }^{140}$ J. Hauser, ${ }^{140}$ M. Ignatenko, ${ }^{140}$ N. Mccoll, ${ }^{140}$ S. Regnard, ${ }^{140}$ D. Saltzberg, ${ }^{140}$ C. Schnaible, ${ }^{140}$ V. Valuev, ${ }^{140}$ E. Bouvier, ${ }^{141}$ K. Burt, ${ }^{141}$ R. Clare, ${ }^{141}$ J. W. Gary, ${ }^{141}$ S. M. A. Ghiasi Shirazi, ${ }^{141}$ G. Hanson, ${ }^{141}$ G. Karapostoli, ${ }^{141}$ E. Kennedy, ${ }^{141}$ F. Lacroix, ${ }^{141}$ O. R. Long, ${ }^{141}$ M. Olmedo Negrete, ${ }^{141}$ M. I. Paneva, ${ }^{141}$ W. Si, ${ }^{141}$ L. Wang, ${ }^{141}$ H. Wei, ${ }^{141}$ S. Wimpenny, ${ }^{141}$ B. R. Yates, ${ }^{141}$ J. G. Branson, ${ }^{142}$ P. Chang, ${ }^{142}$ S. Cittolin, ${ }^{142}$ M. Derdzinski, ${ }^{142}$ R. Gerosa, ${ }^{142}$ D. Gilbert, ${ }^{142}$ B. Hashemi, ${ }^{142}$ A. Holzner, ${ }^{142}$ D. Klein, ${ }^{142}$ G. Kole, ${ }^{142}$ V. Krutelyov, ${ }^{142}$ J. Letts, ${ }^{142}$ M. Masciovecchio, ${ }^{142}$ D. Olivito, ${ }^{142}$ S. Padhi, ${ }^{142}$ M. Pieri, ${ }^{142}$ M. Sani, ${ }^{142}$ V. Sharma, ${ }^{142}$ S. Simon, ${ }^{142}$ M. Tadel, ${ }^{142}$ A. Vartak, ${ }^{142}$ S. Wasserbaech, ${ }^{142, r r r}$ J. Wood, ${ }^{142}$ F. Würthwein, ${ }^{142}$ A. Yagil, ${ }^{142}$ G. Zevi Della Porta, ${ }^{142}$ N. Amin, ${ }^{143}$ R. Bhandari, ${ }^{143}$ J. Bradmiller-Feld, ${ }^{143}$ C. Campagnari, ${ }^{143}$ M. Citron, ${ }^{143}$ A. Dishaw, ${ }^{143}$ V. Dutta, ${ }^{143}$ M. Franco Sevilla, ${ }^{143}$ L. Gouskos, ${ }^{143}$ R. Heller, ${ }^{143}$ J. Incandela, ${ }^{143}$ A. Ovcharova, ${ }^{143}$ H. Qu, ${ }^{143}$ J. Richman, ${ }^{143}$ D. Stuart, ${ }^{143}$ I. Suarez, ${ }^{143}$ S. Wang, ${ }^{143}$ J. Yoo, ${ }^{143}$ D. Anderson, ${ }^{144}$ A. Bornheim, ${ }^{144}$ J. M. Lawhorn, ${ }^{144}$ H. B. Newman, ${ }^{144}$ T. Q. Nguyen, ${ }^{144}$ M. Spiropulu, ${ }^{144}$ J. R. Vlimant, ${ }^{144}$ R. Wilkinson, ${ }^{144}$ S. Xie, ${ }^{144}$ Z. Zhang, ${ }^{144}$ R. Y. Zhu, ${ }^{144}$ M. B. Andrews, ${ }^{145}$ T. Ferguson, ${ }^{145}$ T. Mudholkar, ${ }^{145}$ M. Paulini, ${ }^{145}$ M. Sun, ${ }^{145}$ I. Vorobiev, ${ }^{145}$ M. Weinberg, ${ }^{145}$ J. P. Cumalat, ${ }^{146}$ W. T. Ford, ${ }^{146}$ F. Jensen, ${ }^{146}$ A. Johnson, ${ }^{146}$ M. Krohn, ${ }^{146}$ E. MacDonald, ${ }^{146}$ T. Mulholland, ${ }^{146}$ R. Patel, ${ }^{146}$ A. Perloff, ${ }^{146}$ K. Stenson, ${ }^{146}$ K. A. Ulmer, ${ }^{146}$ S. R. Wagner, ${ }^{146}$ J. Alexander, ${ }^{147}$ J. Chaves, ${ }^{147}$ Y. Cheng, ${ }^{147}$ J. Chu, ${ }^{147}$ A. Datta, ${ }^{147}$ K. Mcdermott, ${ }^{147}$ N. Mirman, ${ }^{147}$ J. R. Patterson, ${ }^{147}$ D. Quach, ${ }^{147}$ A. Rinkevicius, ${ }^{147}$ A. Ryd, ${ }^{147}$ L. Skinnari, ${ }^{147}$ L. Soffi, ${ }^{147}$ S. M. Tan, ${ }^{147}$ Z. Tao, ${ }^{147}$ J. Thom, ${ }^{147}$ J. Tucker, ${ }^{147}$ P. Wittich, ${ }^{147}$ M. Zientek, ${ }^{147}$ S. Abdullin, ${ }^{148}$ M. Albrow, ${ }^{148}$ M. Alyari, ${ }^{148}$ G. Apollinari, ${ }^{148}$ A. Apresyan, ${ }^{148}$ A. Apyan, ${ }^{148}$ S. Banerjee, ${ }^{148}$ L. A. T. Bauerdick, ${ }^{148}$ A. Beretvas, ${ }^{148}$ J. Berryhill, ${ }^{148}$ P. C. Bhat, ${ }^{148}$ K. Burkett, ${ }^{148}$ J. N. Butler, ${ }^{148}$ A. Canepa, ${ }^{148}$ G. B. Cerati, ${ }^{148}$ H. W. K. Cheung, ${ }^{148}$ F. Chlebana, ${ }^{148}$ M. Cremonesi, ${ }^{148}$ J. Duarte, ${ }^{148}$ V. D. Elvira, ${ }^{148}$ J. Freeman, ${ }^{148}$ Z. Gecse, ${ }^{148}$ E. Gottschalk, ${ }^{148}$ L. Gray, ${ }^{148}$ D. Green, ${ }^{148}$ S. Grünendahl, ${ }^{148}$ O. Gutsche, ${ }^{148}$ J. Hanlon, ${ }^{148}$ R. M. Harris, ${ }^{148}$ S. Hasegawa, ${ }^{148}$ J. Hirschauer, ${ }^{148}$ Z. Hu, ${ }^{148}$ B. Jayatilaka, ${ }^{148}$ S. Jindariani, ${ }^{148}$ M. Johnson, ${ }^{148}$ U. Joshi, ${ }^{148}$ B. Klima, ${ }^{148}$ M. J. Kortelainen, ${ }^{148}$ B. Kreis, ${ }^{148}$ S. Lammel, ${ }^{148}$ D. Lincoln, ${ }^{148}$ R. Lipton, ${ }^{148}$ M. Liu, ${ }^{148}$ T. Liu, ${ }^{148}$ J. Lykken, ${ }^{148}$ K. Maeshima, ${ }^{148}$ J. M. Marraffino, ${ }^{148}$ D. Mason, ${ }^{148}$ P. McBride, ${ }^{148}$ P. Merkel, ${ }^{148}$

S. Mrenna, ${ }^{148}$ S. Nahn, ${ }^{148}$ V. O’Dell, ${ }^{148}$ K. Pedro, ${ }^{148}$ C. Pena, ${ }^{148}$ O. Prokofyev, ${ }^{148}$ G. Rakness, ${ }^{148}$ L. Ristori, ${ }^{148}$ A. Savoy-Navarro, ${ }^{148, \text { sss }}$ B. Schneider, ${ }^{148}$ E. Sexton-Kennedy, ${ }^{148}$ A. Soha, ${ }^{148}$ W. J. Spalding, ${ }^{148}$ L. Spiegel, ${ }^{148}$ S. Stoynev, ${ }^{148}$ J. Strait, ${ }^{148}$ N. Strobbe, ${ }^{148}$ L. Taylor, ${ }^{148}$ S. Tkaczyk, ${ }^{148}$ N. V. Tran, ${ }^{148}$ L. Uplegger, ${ }^{148}$ E. W. Vaandering, ${ }^{148}$ C. Vernieri, ${ }^{148}$ M. Verzocchi, ${ }^{148}$ R. Vidal, ${ }^{148}$ M. Wang, ${ }^{148}$ H. A. Weber, ${ }^{148}$ A. Whitbeck, ${ }^{148}$ D. Acosta, ${ }^{149}$ P. Avery, ${ }^{149}$ P. Bortignon, ${ }^{149}$ D. Bourilkov, ${ }^{149}$ A. Brinkerhoff, ${ }^{149}$ L. Cadamuro, ${ }^{149}$ A. Carnes, ${ }^{149}$ D. Curry, ${ }^{149}$ R. D. Field, ${ }^{149}$ S. V. Gleyzer, ${ }^{149}$

B. M. Joshi, ${ }^{149}$ J. Konigsberg, ${ }^{149}$ A. Korytov, ${ }^{149}$ K. H. Lo, ${ }^{149}$ P. Ma, ${ }^{149}$ K. Matchev, ${ }^{149}$ H. Mei, ${ }^{149}$ G. Mitselmakher, ${ }^{149}$ D. Rosenzweig, ${ }^{149}$ K. Shi, ${ }^{149}$ D. Sperka, ${ }^{149}$ J. Wang, ${ }^{149}$ S. Wang, ${ }^{149}$ X. Zuo, ${ }^{149}$ Y. R. Joshi, ${ }^{150}$ S. Linn, ${ }^{150}$ A. Ackert, ${ }^{151}$ T. Adams, ${ }^{151}$ A. Askew, ${ }^{151}$ S. Hagopian, ${ }^{151}$ V. Hagopian, ${ }^{151}$ K. F. Johnson, ${ }^{151}$ T. Kolberg, ${ }^{151}$ G. Martinez, ${ }^{151}$ T. Perry, ${ }^{151}$ H. Prosper, ${ }^{151}$ A. Saha, ${ }^{151}$ C. Schiber, ${ }^{151}$ R. Yohay, ${ }^{151}$ M. M. Baarmand, ${ }^{152}$ V. Bhopatkar, ${ }^{152}$ S. Colafranceschi, ${ }^{152}$ M. Hohlmann, ${ }^{152}$ D. Noonan, ${ }^{152}$ M. Rahmani, ${ }^{152}$ T. Roy, ${ }^{152}$ F. Yumiceva, ${ }^{152}$ M. R. Adams, ${ }^{153}$ L. Apanasevich, ${ }^{153}$ D. Berry, ${ }^{153}$ R. R. Betts, ${ }^{153}$ R. Cavanaugh, ${ }^{153}$ X. Chen, ${ }^{153}$ S. Dittmer, ${ }^{153}$ O. Evdokimov, ${ }^{153}$ C. E. Gerber, ${ }^{153}$ D. A. Hangal, ${ }^{153}$ D. J. Hofman, ${ }^{153}$ K. Jung, ${ }^{153}$ J. Kamin, ${ }^{153}$ C. Mills, ${ }^{153}$ I. D. Sandoval Gonzalez, ${ }^{153}$ M. B. Tonjes, ${ }^{153}$ H. Trauger, ${ }^{153}$ N. Varelas, ${ }^{153}$ H. Wang, ${ }^{153}$ X. Wang, ${ }^{153}$ Z. Wu, ${ }^{153}$ J. Zhang, ${ }^{153}$ M. Alhusseini, ${ }^{154}$ B. Bilki, ${ }^{154, t t t}$ W. Clarida, ${ }^{154}$ K. Dilsiz, ${ }^{154, \text { uuu }}$ S. Durgut, ${ }^{154}$ R. P. Gandrajula, ${ }^{154}$ M. Haytmyradov, ${ }^{154}$ V. Khristenko, ${ }^{154}$ J.-P. Merlo, ${ }^{154}$ A. Mestvirishvili, ${ }^{154}$ A. Moeller, ${ }^{154}$ J. Nachtman, ${ }^{154}$ H. Ogul, ${ }^{154, \mathrm{vvv}}$ Y. Onel, ${ }^{154}$ F. Ozok, ${ }^{154, w w w}$ A. Penzo, ${ }^{154}$ C. Snyder, ${ }^{154}$ E. Tiras, ${ }^{154}$ J. Wetzel, ${ }^{154}$ B. Blumenfeld, ${ }^{155}$ A. Cocoros, ${ }^{155}$ N. Eminizer, ${ }^{155}$ D. Fehling, ${ }^{155}$ L. Feng, ${ }^{155}$ A. V. Gritsan, ${ }^{155}$ W. T. Hung, ${ }^{155}$ P. Maksimovic, ${ }^{155}$ J. Roskes, ${ }^{155}$ U. Sarica, ${ }^{155}$ M. Swartz, ${ }^{155}$ M. Xiao, ${ }^{155}$ C. You, ${ }^{155}$ A. Al-bataineh, ${ }^{156}$ P. Baringer, ${ }^{156}$ A. Bean, ${ }^{156}$ S. Boren, ${ }^{156}$ J. Bowen, ${ }^{156}$ A. Bylinkin, ${ }^{156}$ J. Castle, ${ }^{156}$ S. Khalil, ${ }^{156}$ A. Kropivnitskaya, ${ }^{156}$ D. Majumder, ${ }^{156}$ W. Mcbrayer, ${ }^{156}$ M. Murray, ${ }^{156}$ C. Rogan, ${ }^{156}$ S. Sanders, ${ }^{156}$ E. Schmitz, ${ }^{156}$ J. D. Tapia Takaki, ${ }^{156}$ Q. Wang, ${ }^{156}$ S. Duric, ${ }^{157}$ 
A. Ivanov, ${ }^{157}$ K. Kaadze, ${ }^{157}$ D. Kim, ${ }^{157}$ Y. Maravin, ${ }^{157}$ D. R. Mendis, ${ }^{157}$ T. Mitchell, ${ }^{157}$ A. Modak, ${ }^{157}$ A. Mohammadi, ${ }^{157}$ L. K. Saini, ${ }^{157}$ N. Skhirtladze, ${ }^{157}$ F. Rebassoo, ${ }^{158}$ D. Wright, ${ }^{158}$ A. Baden, ${ }^{159}$ O. Baron, ${ }^{159}$ A. Belloni, ${ }^{159}$ S. C. Eno, ${ }^{159}$ Y. Feng, ${ }^{159}$ C. Ferraioli, ${ }^{159}$ N. J. Hadley, ${ }^{159}$ S. Jabeen, ${ }^{159}$ G. Y. Jeng, ${ }^{159}$ R. G. Kellogg, ${ }^{159}$ J. Kunkle, ${ }^{159}$ A. C. Mignerey, ${ }^{159}$ S. Nabili, ${ }^{159}$ F. Ricci-Tam, ${ }^{159}$ Y. H. Shin, ${ }^{159}$ A. Skuja, ${ }^{159}$ S. C. Tonwar, ${ }^{159}$ K. Wong, ${ }^{159}$ D. Abercrombie, ${ }^{160}$ B. Allen, ${ }^{160}$ V. Azzolini, ${ }^{160}$ A. Baty, ${ }^{160}$ G. Bauer, ${ }^{160}$ R. Bi, ${ }^{160}$ S. Brandt, ${ }^{160}$ W. Busza, ${ }^{160}$ I. A. Cali, ${ }^{160}$ M. D' Alfonso, ${ }^{160}$ Z. Demiragli, ${ }^{160}$ G. Gomez Ceballos, ${ }^{160}$ M. Goncharov, ${ }^{160}$ P. Harris, ${ }^{160}$ D. Hsu, ${ }^{160}$ M. Hu, ${ }^{160}$ Y. Iiyama, ${ }^{160}$ G. M. Innocenti, ${ }^{160}$ M. Klute, ${ }^{160}$ D. Kovalskyi, ${ }^{160}$ Y.-J. Lee, ${ }^{160}$ P. D. Luckey, ${ }^{160}$ B. Maier, ${ }^{160}$ A. C. Marini, ${ }^{160}$ C. Mcginn, ${ }^{160}$ C. Mironov, ${ }^{160}$ S. Narayanan, ${ }^{160}$ X. Niu, ${ }^{160}$ C. Paus, ${ }^{160}$ C. Roland, ${ }^{160}$ G. Roland, ${ }^{160}$ G. S. F. Stephans, ${ }^{160}$ K. Sumorok, ${ }^{160}$ K. Tatar, ${ }^{160}$ D. Velicanu, ${ }^{160}$ J. Wang, ${ }^{160}$ T. W. Wang, ${ }^{160}$ B. Wyslouch, ${ }^{160}$ S. Zhaozhong, ${ }^{160}$ A. C. Benvenuti, ${ }^{161, a}$ R. M. Chatterjee, ${ }^{161}$ A. Evans, ${ }^{161}$ P. Hansen, ${ }^{161}$ J. Hiltbrand, ${ }^{161}$ Sh. Jain, ${ }^{161}$ S. Kalafut, ${ }^{161}$ Y. Kubota, ${ }^{161}$ Z. Lesko, ${ }^{161}$ J. Mans, ${ }^{161}$ N. Ruckstuhl, ${ }^{161}$ R. Rusack, ${ }^{161}$ M. A. Wadud, ${ }^{161}$ J. G. Acosta, ${ }^{162}$ S. Oliveros, ${ }^{162}$ E. Avdeeva, ${ }^{163}$ K. Bloom, ${ }^{163}$ D. R. Claes, ${ }^{163}$ C. Fangmeier, ${ }^{163}$ F. Golf, ${ }^{163}$ R. Gonzalez Suarez, ${ }^{163}$ R. Kamalieddin, ${ }^{163}$ I. Kravchenko, ${ }^{163}$ J. Monroy, ${ }^{163}$ J. E. Siado, ${ }^{163}$ G. R. Snow, ${ }^{163}$ B. Stieger, ${ }^{163}$ A. Godshalk, ${ }^{164}$ C. Harrington, ${ }^{164}$ I. Iashvili, ${ }^{164}$ A. Kharchilava, ${ }^{164}$ C. Mclean, ${ }^{164}$ D. Nguyen, ${ }^{164}$ A. Parker, ${ }^{164}$ S. Rappoccio, ${ }^{164}$ B. Roozbahani, ${ }^{164}$ G. Alverson, ${ }^{165}$ E. Barberis, ${ }^{165}$ C. Freer, ${ }^{165}$ Y. Haddad, ${ }^{165}$ A. Hortiangtham, ${ }^{165}$ D. M. Morse, ${ }^{165}$ T. Orimoto, ${ }^{165}$ R. Teixeira De Lima, ${ }^{165}$ T. Wamorkar, ${ }^{165}$ B. Wang, ${ }^{165}$ A. Wisecarver, ${ }^{165}$ D. Wood, ${ }^{165}$ S. Bhattacharya, ${ }^{166}$ O. Charaf, ${ }^{166}$ K. A. Hahn, ${ }^{166}$ N. Mucia, ${ }^{166}$ N. Odell, ${ }^{166}$ M. H. Schmitt,,${ }^{166}$ K. Sung, ${ }^{166}$ M. Trovato, ${ }^{166}$ M. Velasco, ${ }^{166}$ R. Bucci, ${ }^{167}$ N. Dev ${ }^{167}$ M. Hildreth,${ }^{167}$ K. Hurtado Anampa ${ }^{167}$ C. Jessop, ${ }^{167}$ D. J. Karmgard, ${ }^{167}$ N. Kellams, ${ }^{167}$ K. Lannon, ${ }^{167}$ W. Li,${ }^{167}$ N. Loukas, ${ }^{167}$ N. Marinelli, ${ }^{167}$ F. Meng, ${ }^{167}$ C. Mueller, ${ }^{167}$ Y. Musienko, ${ }^{167, m m}$ M. Planer, ${ }^{167}$ A. Reinsvold, ${ }^{167}$ R. Ruchti, ${ }^{167}$ P. Siddireddy, ${ }^{167}$ G. Smith, ${ }^{167}$ S. Taroni, ${ }^{167}$ M. Wayne, ${ }^{167}$ A. Wightman, ${ }^{167}$ M. Wolf, ${ }^{167}$ A. Woodard, ${ }^{167}$ J. Alimena, ${ }^{168}$ L. Antonelli, ${ }^{168}$ B. Bylsma, ${ }^{168}$ L. S. Durkin, ${ }^{168}$ S. Flowers, ${ }^{168}$ B. Francis, ${ }^{168}$ C. Hill, ${ }^{168}$ W. Ji, ${ }^{168}$ T. Y. Ling, ${ }^{168}$ W. Luo, ${ }^{168}$ B. L. Winer, ${ }^{168}$ S. Cooperstein, ${ }^{169}$ P. Elmer, ${ }^{169}$ J. Hardenbrook, ${ }^{169}$ S. Higginbotham, ${ }^{169}$ A. Kalogeropoulos, ${ }^{169}$ D. Lange, ${ }^{169}$ M. T. Lucchini, ${ }^{169}$ J. Luo, ${ }^{169}$ D. Marlow, ${ }^{169}$ K. Mei ${ }^{169}$ I. Ojalvo, ${ }^{169}$ J. Olsen, ${ }^{169}$ C. Palmer, ${ }^{169}$ P. Piroué, ${ }^{169}$ J. Salfeld-Nebgen, ${ }^{169}$ D. Stickland, ${ }^{169}$ C. Tully, ${ }^{169}$ S. Malik ${ }^{170}$ S. Norberg, ${ }^{170}$ A. Barker, ${ }^{171}$ V. E. Barnes, ${ }^{171}$ S. Das, ${ }^{171}$ L. Gutay, ${ }^{171}$ M. Jones, ${ }^{171}$ A. W. Jung, ${ }^{171}$ A. Khatiwada, ${ }^{171}$ B. Mahakud, ${ }^{171}$ D. H. Miller, ${ }^{171}$ N. Neumeister, ${ }^{171}$ C. C. Peng, ${ }^{171}$ S. Piperov, ${ }^{171}$ H. Qiu, ${ }^{171}$ J. F. Schulte, ${ }^{171}$ J. Sun, ${ }^{171}$ F. Wang, ${ }^{171}$ R. Xiao, ${ }^{171}$ W. Xie, ${ }^{171}$ T. Cheng, ${ }^{172}$ J. Dolen, ${ }^{172}$ N. Parashar, ${ }^{172}$ Z. Chen, ${ }^{173}$ K. M. Ecklund, ${ }^{173}$ S. Freed, ${ }^{173}$ F. J. M. Geurts, ${ }^{173}$ M. Kilpatrick, ${ }^{173}$ W. Li, ${ }^{173}$ B. P. Padley ${ }^{173}$ J. Roberts, ${ }^{173}$ J. Rorie, ${ }^{173}$ W. Shi ${ }^{173}$ Z. Tu,${ }^{173}$ A. Zhang, ${ }^{173}$ A. Bodek, ${ }^{174}$ P. de Barbaro, ${ }^{174}$ R. Demina, ${ }^{174}$ Y. t. Duh, ${ }^{174}$ J. L. Dulemba, ${ }^{174}$ C. Fallon, ${ }^{174}$ T. Ferbel, ${ }^{174}$ M. Galanti, ${ }^{174}$ A. Garcia-Bellido, ${ }^{174}$ J. Han, ${ }^{174}$ O. Hindrichs, ${ }^{174}$ A. Khukhunaishvili, ${ }^{174}$ P. Tan, ${ }^{174}$ R. Taus, ${ }^{174}$ A. Agapitos, ${ }^{175}$ J. P. Chou, ${ }^{175}$ Y. Gershtein, ${ }^{175}$ E. Halkiadakis, ${ }^{175}$ A. Hart, ${ }^{175}$ M. Heindl, ${ }^{175}$ E. Hughes, ${ }^{175}$ S. Kaplan, ${ }^{175}$ R. Kunnawalkam Elayavalli, ${ }^{175}$ S. Kyriacou, ${ }^{175}$ A. Lath, ${ }^{175}$ R. Montalvo, ${ }^{175}$ K. Nash ${ }^{175}$ M. Osherson, ${ }^{175}$ H. Saka, ${ }^{175}$ S. Salur, ${ }^{175}$ S. Schnetzer, ${ }^{175}$ D. Sheffield, ${ }^{175}$ S. Somalwar, ${ }^{175}$ R. Stone ${ }^{175}$ S. Thomas, ${ }^{175}$ P. Thomassen, ${ }^{175}$ M. Walker, ${ }^{175}$ A. G. Delannoy, ${ }^{176}$ J. Heideman,${ }^{176}$ G. Riley, ${ }^{176}$ S. Spanier, ${ }^{176}$ O. Bouhali, ${ }^{177, x x x}$ A. Celik, ${ }^{177}$ M. Dalchenko, ${ }^{177}$ M. De Mattia, ${ }^{177}$ A. Delgado, ${ }^{177}$ S. Dildick, ${ }^{177}$ R. Eusebi, ${ }^{177}$ J. Gilmore, ${ }^{177}$ T. Huang, ${ }^{177}$ T. Kamon, ${ }^{177, y y y}$ S. Luo, ${ }^{177}$ R. Mueller, ${ }^{177}$ D. Overton, ${ }^{177}$ L. Perniè, ${ }^{177}$ D. Rathjens, ${ }^{177}$ A. Safonov, ${ }^{177}$ N. Akchurin, ${ }^{178}$ J. Damgov, ${ }^{178}$ F. De Guio, ${ }^{178}$ P. R. Dudero, ${ }^{178}$ S. Kunori, ${ }^{178}$ K. Lamichhane,${ }^{178}$ S. W. Lee, ${ }^{178}$ T. Mengke, ${ }^{178}$ S. Muthumuni, ${ }^{178}$ T. Peltola, ${ }^{178}$ S. Undleeb, ${ }^{178}$ I. Volobouev, ${ }^{178}$ Z. Wang, ${ }^{178}$ S. Greene, ${ }^{179}$ A. Gurrola, ${ }^{179}$ R. Janjam, ${ }^{179}$ W. Johns,${ }^{179}$ C. Maguire,${ }^{179}$ A. Melo, ${ }^{179}$ H. Ni, ${ }^{179}$ K. Padeken, ${ }^{179}$ J. D. Ruiz Alvarez, ${ }^{179}$ P. Sheldon, ${ }^{179}$ S. Tuo, ${ }^{179}$ J. Velkovska, ${ }^{179}$ M. Verweij, ${ }^{179}$ Q. Xu, ${ }^{179}$ M. W. Arenton, ${ }^{100}$ P. Barria, ${ }^{180}$ B. Cox ${ }^{180}$ R. Hirosky, ${ }^{180}$ M. Joyce, ${ }^{180}$ A. Ledovskoy, ${ }_{180}^{18}$ H. Li,${ }^{180}$ C. Neu ${ }^{180}$ T. Sinthuprasith, ${ }^{180}$ Y. Wang,${ }^{180}$ E. Wolfe, ${ }^{180}$ F. Xia, ${ }^{180}$ R. Harr, ${ }^{181}$ P. E. Karchin, ${ }^{181}$ N. Poudyal, ${ }^{181}$ J. Sturdy, ${ }^{181}$ P. Thapa, ${ }^{181}$ S. Zaleski, ${ }^{181}$ M. Brodski, ${ }^{182}$ J. Buchanan, ${ }^{182}$ C. Caillol, ${ }^{182}$ D. Carlsmith ${ }^{182}$

S. Dasu, ${ }^{182}$ L. Dodd ${ }^{182}$ B. Gomber ${ }^{182}$ M. Grothe, ${ }^{182}$ M. Herndon, ${ }^{182}$ A. Hervé, ${ }^{182}$ U. Hussain, ${ }^{182}$ P. Klabbers, ${ }^{182}$ A. Lanaro, ${ }^{182}$ K. Long, ${ }^{182}$ R. Loveless, ${ }^{182}$ T. Ruggles, ${ }^{182}$ A. Savin, ${ }^{182}$ V. Sharma, ${ }^{182}$ N. Smith, ${ }^{182}$ W. H. Smith, ${ }^{182}$ and N. Woods ${ }^{182}$

(CMS Collaboration)

\footnotetext{
${ }^{1}$ Yerevan Physics Institute, Yerevan, Armenia

${ }_{2}^{2}$ Institut für Hochenergiephysik, Wien, Austria

${ }^{3}$ Institute for Nuclear Problems, Minsk, Belarus

${ }^{4}$ Universiteit Antwerpen, Antwerpen, Belgium
} 


\author{
${ }^{5}$ Vrije Universiteit Brussel, Brussel, Belgium \\ ${ }^{6}$ Université Libre de Bruxelles, Bruxelles, Belgium \\ ${ }^{7}$ Ghent University, Ghent, Belgium \\ ${ }^{8}$ Université Catholique de Louvain, Louvain-la-Neuve, Belgium \\ ${ }^{9}$ Centro Brasileiro de Pesquisas Fisicas, Rio de Janeiro, Brazil \\ ${ }^{10}$ Universidade do Estado do Rio de Janeiro, Rio de Janeiro, Brazil \\ ${ }^{11 a}$ Universidade Estadual Paulista, São Paulo, Brazil \\ ${ }^{11 \mathrm{~b}}$ Universidade Federal do ABC, São Paulo, Brazil \\ ${ }^{12}$ Institute for Nuclear Research and Nuclear Energy, Bulgarian Academy of Sciences, Sofia, Bulgaria \\ ${ }^{13}$ University of Sofia, Sofia, Bulgaria \\ ${ }^{14}$ Beihang University, Beijing, China \\ ${ }^{15}$ Institute of High Energy Physics, Beijing, China \\ ${ }^{16}$ State Key Laboratory of Nuclear Physics and Technology, Peking University, Beijing, China \\ ${ }^{17}$ Tsinghua University, Beijing, China \\ ${ }^{18}$ Universidad de Los Andes, Bogota, Colombia \\ ${ }^{19}$ University of Split, Faculty of Electrical Engineering, Mechanical Engineering and Naval Architecture, \\ Split, Croatia \\ ${ }^{20}$ University of Split, Faculty of Science, Split, Croatia \\ ${ }^{21}$ Institute Rudjer Boskovic, Zagreb, Croatia \\ ${ }^{22}$ University of Cyprus, Nicosia, Cyprus \\ ${ }^{23}$ Charles University, Prague, Czech Republic \\ ${ }^{24}$ Escuela Politecnica Nacional, Quito, Ecuador \\ ${ }^{25}$ Universidad San Francisco de Quito, Quito, Ecuador \\ ${ }^{26}$ Academy of Scientific Research and Technology of the Arab Republic of Egypt, \\ Egyptian Network of High Energy Physics, Cairo, Egypt \\ ${ }^{27}$ National Institute of Chemical Physics and Biophysics, Tallinn, Estonia \\ ${ }^{28}$ Department of Physics, University of Helsinki, Helsinki, Finland \\ ${ }^{29}$ Helsinki Institute of Physics, Helsinki, Finland \\ ${ }^{30}$ Lappeenranta University of Technology, Lappeenranta, Finland \\ ${ }^{31}$ IRFU, CEA, Université Paris-Saclay, Gif-sur-Yvette, France \\ ${ }^{32}$ Laboratoire Leprince-Ringuet, Ecole polytechnique, CNRS/IN2P3, Université Paris-Saclay, \\ Palaiseau, France \\ ${ }^{33}$ Université de Strasbourg, CNRS, IPHC UMR 7178, Strasbourg, France \\ ${ }^{34}$ Centre de Calcul de l'Institut National de Physique Nucleaire et de Physique des Particules, \\ CNRS/IN2P3, Villeurbanne, France \\ ${ }^{35}$ Université de Lyon, Université Claude Bernard Lyon 1, CNRS-IN2P3, \\ Institut de Physique Nucléaire de Lyon, Villeurbanne, France \\ ${ }^{36}$ Georgian Technical University, Tbilisi, Georgia \\ ${ }^{37}$ Tbilisi State University, Tbilisi, Georgia \\ ${ }^{38}$ RWTH Aachen University, I. Physikalisches Institut, Aachen, Germany \\ ${ }^{39}$ RWTH Aachen University, III. Physikalisches Institut A, Aachen, Germany \\ ${ }^{40}$ RWTH Aachen University, III. Physikalisches Institut B, Aachen, Germany \\ ${ }^{41}$ Deutsches Elektronen-Synchrotron, Hamburg, Germany \\ ${ }^{42}$ University of Hamburg, Hamburg, Germany \\ ${ }^{43}$ Karlsruher Institut fuer Technologie, Karlsruhe, Germany \\ ${ }^{44}$ Institute of Nuclear and Particle Physics (INPP), NCSR Demokritos, Aghia Paraskevi, Greece \\ ${ }^{45}$ National and Kapodistrian University of Athens, Athens, Greece \\ ${ }^{46}$ National Technical University of Athens, Athens, Greece \\ ${ }^{47}$ University of Ioánnina, Ioánnina, Greece \\ ${ }^{48}$ MTA-ELTE Lendület CMS Particle and Nuclear Physics Group, Eötvös Loránd University, \\ Budapest, Hungary \\ ${ }^{49}$ Wigner Research Centre for Physics, Budapest, Hungary \\ ${ }^{50}$ Institute of Nuclear Research ATOMKI, Debrecen, Hungary \\ ${ }^{51}$ Institute of Physics, University of Debrecen, Debrecen, Hungary \\ ${ }^{52}$ Indian Institute of Science (IISc), Bangalore, India \\ ${ }^{53}$ National Institute of Science Education and Research, HBNI, Bhubaneswar, India \\ ${ }^{54}$ Panjab University, Chandigarh, India \\ ${ }^{55}$ University of Delhi, Delhi, India \\ ${ }^{56}$ Saha Institute of Nuclear Physics, HBNI, Kolkata, India \\ ${ }^{57}$ Indian Institute of Technology Madras, Madras, India
}




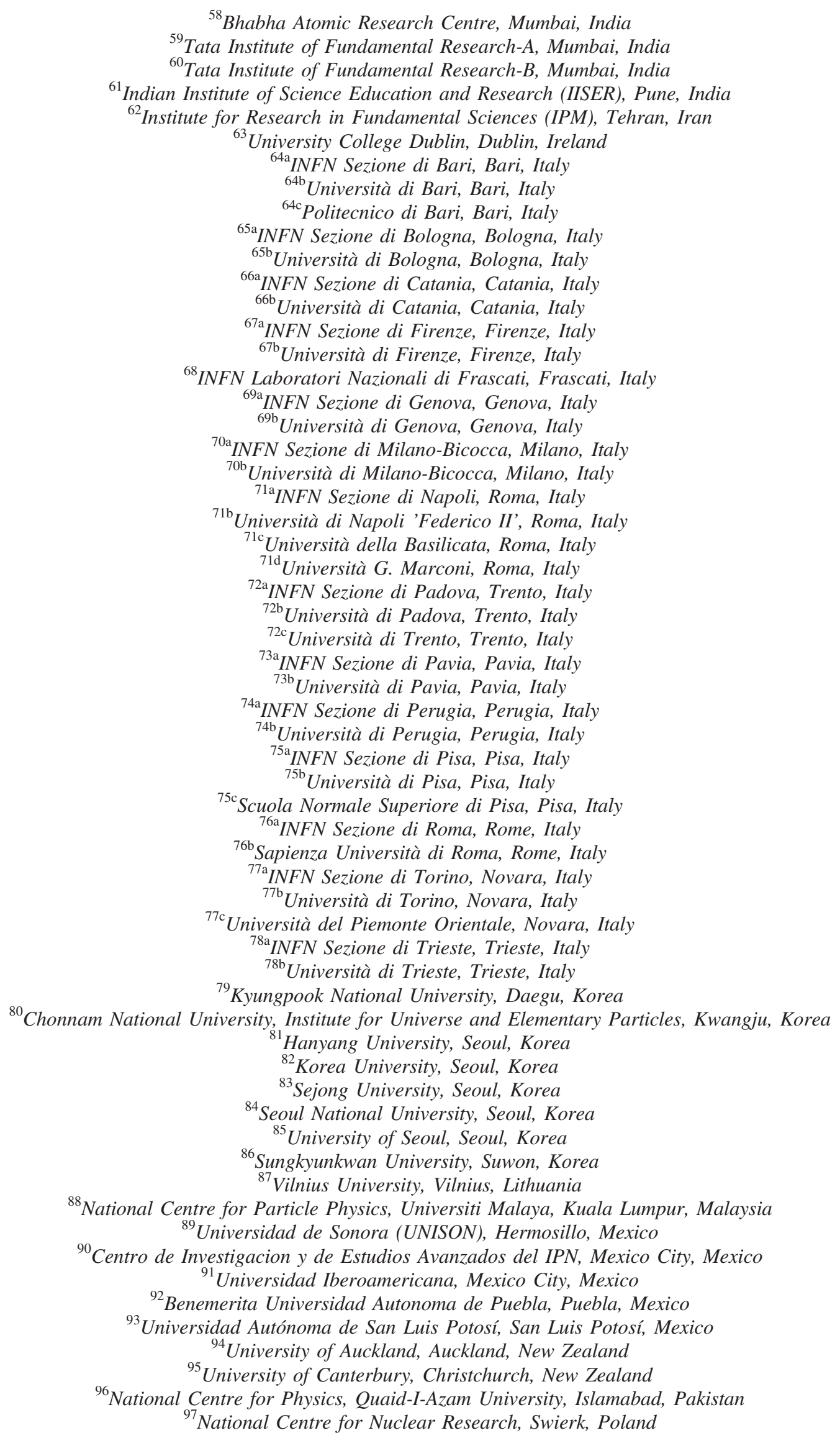


${ }^{98}$ Institute of Experimental Physics, Faculty of Physics, University of Warsaw, Warsaw, Poland

${ }^{99}$ Laboratório de Instrumentação e Física Experimental de Partículas, Lisboa, Portugal

${ }^{100}$ Joint Institute for Nuclear Research, Dubna, Russia

${ }^{101}$ Petersburg Nuclear Physics Institute, Gatchina (St. Petersburg), Russia

${ }^{102}$ Institute for Nuclear Research, Moscow, Russia

${ }^{103}$ Institute for Theoretical and Experimental Physics, Moscow, Russia

${ }^{104}$ Moscow Institute of Physics and Technology, Moscow, Russia

${ }^{105}$ National Research Nuclear University 'Moscow Engineering Physics Institute' (MEPhI), Moscow, Russia

${ }^{106}$ P.N. Lebedev Physical Institute, Moscow, Russia

${ }^{107}$ Skobeltsyn Institute of Nuclear Physics, Lomonosov Moscow State University, Moscow, Russia

${ }^{108}$ Novosibirsk State University (NSU), Novosibirsk, Russia

${ }^{109}$ Institute for High Energy Physics of National Research Centre 'Kurchatov Institute', Protvino, Russia

${ }^{110}$ National Research Tomsk Polytechnic University, Tomsk, Russia

${ }^{111}$ University of Belgrade, Faculty of Physics and Vinca Institute of Nuclear Sciences, Belgrade, Serbia

${ }^{112}$ Centro de Investigaciones Energéticas Medioambientales y Tecnológicas (CIEMAT), Madrid, Spain

${ }^{113}$ Universidad Autónoma de Madrid, Madrid, Spain

${ }^{114}$ Universidad de Oviedo, Oviedo, Spain

${ }^{115}$ Instituto de Física de Cantabria (IFCA), CSIC-Universidad de Cantabria, Santander, Spain

${ }^{116}$ University of Ruhuna, Department of Physics, Matara, Sri Lanka

${ }^{117}$ CERN, European Organization for Nuclear Research, Geneva, Switzerland

${ }^{118}$ Paul Scherrer Institut, Villigen, Switzerland

${ }^{119}$ ETH Zurich-Institute for Particle Physics and Astrophysics (IPA), Zurich, Switzerland

${ }^{120}$ Universität Zürich, Zurich, Switzerland

${ }^{121}$ National Central University, Chung-Li, Taiwan

${ }^{122}$ National Taiwan University (NTU), Taipei, Taiwan

${ }^{123}$ Chulalongkorn University, Faculty of Science, Department of Physics, Bangkok, Thailand

${ }^{124}$ Cukurova University, Physics Department, Science and Art Faculty, Adana, Turkey

${ }^{125}$ Middle East Technical University, Physics Department, Ankara, Turkey

${ }^{126}$ Bogazici University, Istanbul, Turkey

${ }^{127}$ Istanbul Technical University, Istanbul, Turkey

${ }^{128}$ Institute for Scintillation Materials of National Academy of Science of Ukraine, Kharkov, Ukraine

${ }^{129}$ National Scientific Center, Kharkov Institute of Physics and Technology, Kharkov, Ukraine

${ }^{130}$ University of Bristol, Bristol, United Kingdom

${ }^{131}$ Rutherford Appleton Laboratory, Didcot, United Kingdom

${ }^{132}$ Imperial College, London, United Kingdom

${ }^{133}$ Brunel University, Uxbridge, United Kingdom

${ }^{134}$ Baylor University, Waco, Texas, USA

${ }^{135}$ Catholic University of America, Washington, DC, USA

${ }^{136}$ The University of Alabama, Tuscaloosa, Alabama, USA

${ }^{137}$ Boston University, Boston, Massachusetts, USA

${ }^{138}$ Brown University, Providence, Rhode Island, USA

${ }^{139}$ University of California, Davis, Davis, California, USA

${ }^{140}$ University of California, Los Angeles, California, USA

${ }^{141}$ University of California, Riverside, Riverside, California, USA

${ }^{142}$ University of California, San Diego, La Jolla, California, USA

${ }^{143}$ University of California, Santa Barbara-Department of Physics, Santa Barbara, California, USA

${ }^{144}$ California Institute of Technology, Pasadena, California, USA

${ }^{145}$ Carnegie Mellon University, Pittsburgh, Pennsylvania, USA

${ }^{146}$ University of Colorado Boulder, Boulder, Colorado, USA

${ }^{147}$ Cornell University, Ithaca, New York, USA

${ }^{148}$ Fermi National Accelerator Laboratory, Batavia, Illinois, USA

${ }^{149}$ University of Florida, Gainesville, Florida, USA

${ }^{150}$ Florida International University, Miami, Florida, USA

${ }^{151}$ Florida State University, Tallahassee, Florida, USA

${ }^{152}$ Florida Institute of Technology, Melbourne, Florida, USA

${ }^{153}$ University of Illinois at Chicago (UIC), Chicago, Illinois, USA

${ }^{154}$ The University of Iowa, Iowa City, Iowa, USA

${ }^{155}$ Johns Hopkins University, Baltimore, Maryland, USA

${ }^{156}$ The University of Kansas, Lawrence, Kansas, USA 


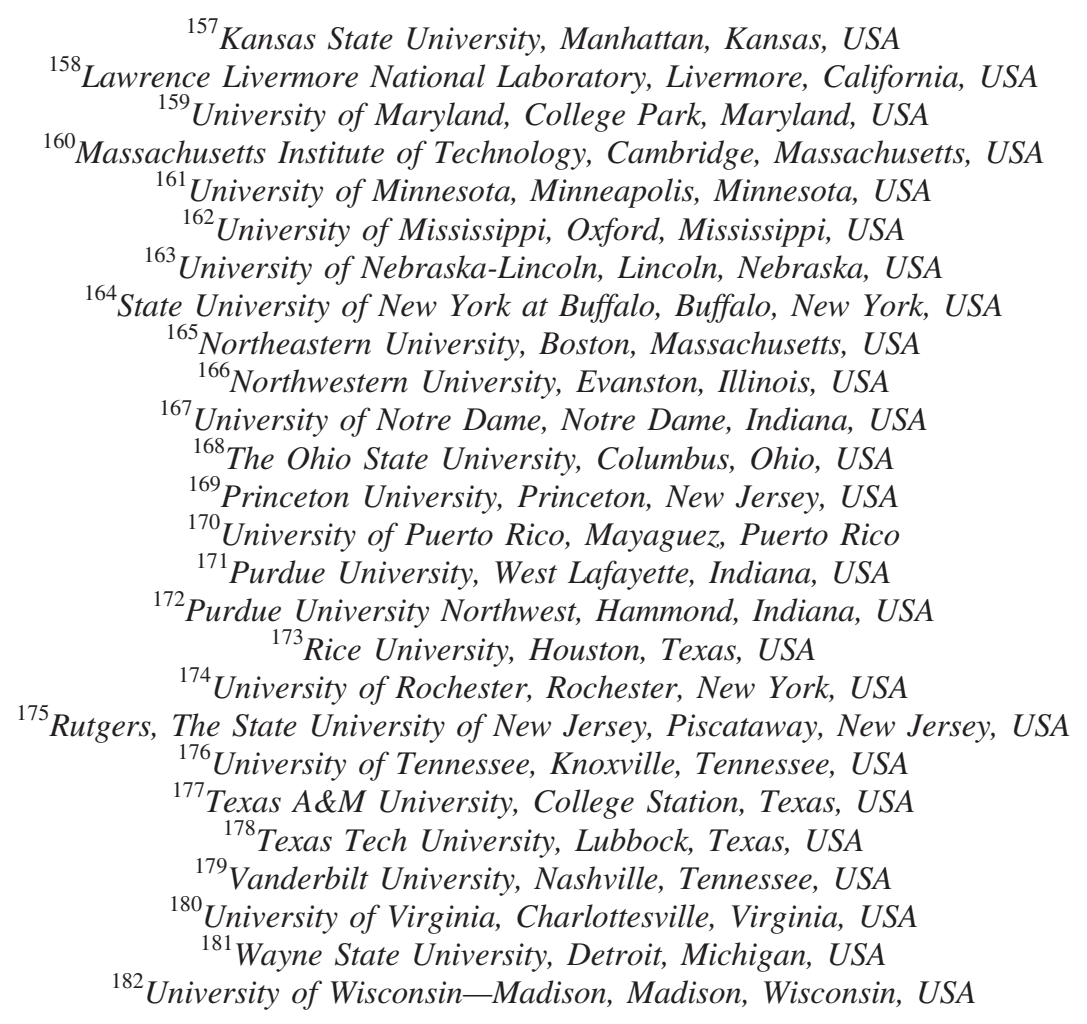

${ }^{\mathrm{a}}$ Deceased.

${ }^{\mathrm{b}}$ Also at Vienna University of Technology, Vienna, Austria.

${ }^{\mathrm{c}}$ Also at IRFU, CEA, Université Paris-Saclay, Gif-sur-Yvette, France.

${ }^{\mathrm{d}}$ Also at Universidade Estadual de Campinas, Campinas, Brazil.

${ }^{\mathrm{e}}$ Also at Federal University of Rio Grande do Sul, Porto Alegre, Brazil.

${ }^{\mathrm{f}}$ Also at Université Libre de Bruxelles, Bruxelles, Belgium.

${ }^{\mathrm{g}}$ Also at University of Chinese Academy of Sciences, Beijing, China.

${ }^{\mathrm{h}}$ Also at Institute for Theoretical and Experimental Physics, Moscow, Russia.

${ }^{\mathrm{i}}$ Also at Joint Institute for Nuclear Research, Dubna, Russia.

${ }^{\mathrm{j}}$ Also at Cairo University, Cairo, Egypt.

${ }^{\mathrm{k}}$ Also at Helwan University, Cairo, Egypt.

${ }^{1}$ Also at Zewail City of Science and Technology, Zewail, Egypt.

${ }^{\mathrm{m}}$ Also at British University in Egypt, Cairo, Egypt.

${ }^{\mathrm{n}}$ Also at Fayoum University, El-Fayoum, Egypt.

${ }^{\circ}$ Also at Department of Physics, King Abdulaziz University, Jeddah, Saudi Arabia.

${ }^{\mathrm{p}}$ Also at Université de Haute Alsace, Mulhouse, France.

${ }^{\mathrm{q}}$ Also at Skobeltsyn Institute of Nuclear Physics, Lomonosov Moscow State University, Moscow, Russia.

${ }^{\mathrm{r}}$ Also at Tbilisi State University, Tbilisi, Georgia.

${ }^{\mathrm{s}}$ Also at CERN, European Organization for Nuclear Research, Geneva, Switzerland.

${ }^{t}$ Also at RWTH Aachen University, III. Physikalisches Institut A, Aachen, Germany.

${ }^{\mathrm{u}}$ Also at University of Hamburg, Hamburg, Germany.

${ }^{\mathrm{v}}$ Also at Brandenburg University of Technology, Cottbus, Germany.

${ }^{\mathrm{w}}$ Also at MTA-ELTE Lendület CMS Particle and Nuclear Physics Group, Eötvös Loránd University, Budapest, Hungary.

${ }^{\mathrm{x}}$ Also at Institute of Nuclear Research ATOMKI, Debrecen, Hungary.

${ }^{y}$ Also at Institute of Physics, University of Debrecen, Debrecen, Hungary.

${ }^{\mathrm{z}}$ Also at IIT Bhubaneswar, Bhubaneswar, India.

${ }^{\text {aa } A l s o ~ a t ~ I n s t i t u t e ~ o f ~ P h y s i c s, ~ B h u b a n e s w a r, ~ I n d i a . ~}$

${ }^{\mathrm{bb}}$ Also at Shoolini University, Solan, India.

${ }^{\mathrm{cc}}$ Also at University of Visva-Bharati, Santiniketan, India.

${ }^{\mathrm{dd}}$ Also at Isfahan University of Technology, Isfahan, Iran.

${ }^{e e}$ Also at Plasma Physics Research Center, Science and Research Branch, Islamic Azad University, Tehran, Iran.

${ }^{\mathrm{ff}}$ Also at Università degli Studi di Siena, Siena, Italy.

${ }^{\text {gg }}$ Also at Scuola Normale e Sezione dell'INFN, Pisa, Italy. 
${ }^{\text {hh }}$ Also at Kyunghee University, Seoul, Korea.

${ }^{\text {ii } A l s o ~ a t ~ I n t e r n a t i o n a l ~ I s l a m i c ~ U n i v e r s i t y ~ o f ~ M a l a y s i a, ~ K u a l a ~ L u m p u r, ~ M a l a y s i a . ~}$

${ }^{\mathrm{jj}}$ Also at Malaysian Nuclear Agency, MOSTI, Kajang, Malaysia.

${ }^{k k}$ Also at Consejo Nacional de Ciencia y Tecnología, Mexico city, Mexico.

${ }^{11}$ Also at Warsaw University of Technology, Institute of Electronic Systems, Warsaw, Poland.

${ }^{\mathrm{mm}}$ Also at Institute for Nuclear Research, Moscow, Russia.

${ }^{n n}$ Also at National Research Nuclear University 'Moscow Engineering Physics Institute' (MEPhI), Moscow, Russia.

${ }^{\text {oo }}$ Also at St. Petersburg State Polytechnical University, St. Petersburg, Russia.

${ }^{\mathrm{pp}}$ Also at University of Florida, Gainesville, Florida, USA.

${ }^{\mathrm{qq}}$ Also at P.N. Lebedev Physical Institute, Moscow, Russia.

${ }^{\text {rr }}$ Also at California Institute of Technology, Pasadena, California, USA.

${ }^{\text {ss }}$ Also at Budker Institute of Nuclear Physics, Novosibirsk, Russia.

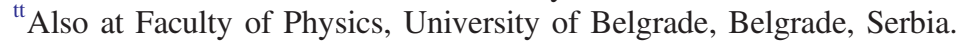

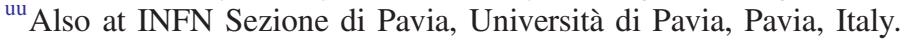

${ }^{\mathrm{vv}}$ Also at University of Belgrade, Faculty of Physics and Vinca Institute of Nuclear Sciences, Belgrade, Serbia.

${ }^{w w}$ Also at National and Kapodistrian University of Athens, Athens, Greece.

${ }^{\mathrm{xx}}$ Also at Riga Technical University, Riga, Latvia.

${ }^{y y}$ Also at Universität Zürich, Zurich, Switzerland.

${ }^{z z}$ Also at Stefan Meyer Institute for Subatomic Physics, Vienna, Austria.

${ }^{\text {aaa }}$ Also at Adiyaman University, Adiyaman, Turkey.

${ }^{b b b}$ Also at Istanbul Aydin University, Istanbul, Turkey.

${ }^{\mathrm{ccc}}$ Also at Mersin University, Mersin, Turkey.

${ }^{\text {ddd }}$ Also at Piri Reis University, Istanbul, Turkey.

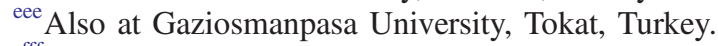

${ }^{\mathrm{fff}}$ Also at Ozyegin University, Istanbul, Turkey.

${ }^{\text {ggg }}$ Also at Izmir Institute of Technology, Izmir, Turkey.

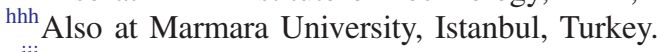

iii Also at Kafkas University, Kars, Turkey.

${ }^{\mathrm{jjj}}$ Also at Istanbul University, Faculty of Science, Istanbul, Turkey.

${ }^{\mathrm{kkk}}$ Also at Istanbul Bilgi University, Istanbul, Turkey.

${ }^{111}$ Also at Hacettepe University, Ankara, Turkey.

${ }^{\mathrm{mmm}}$ Also at Rutherford Appleton Laboratory, Didcot, United Kingdom.

${ }^{n n n}$ Also at School of Physics and Astronomy, University of Southampton, Southampton, United Kingdom.

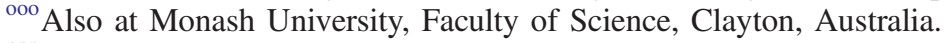

${ }^{\mathrm{ppp}}$ Also at Bethel University, St. Paul, Minnesota, USA.

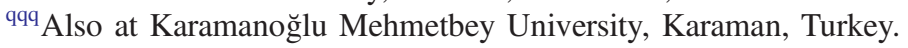

${ }^{\mathrm{rrr}}$ Also at Utah Valley University, Orem, Utah, USA.

${ }^{\text {sss }}$ Also at Purdue University, West Lafayette, Indiana, USA.

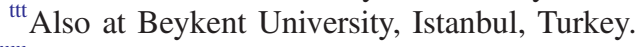

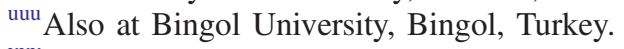

${ }^{\mathrm{vvv}}$ Also at Sinop University, Sinop, Turkey.

${ }^{w w w}$ Also at Mimar Sinan University, Istanbul, Istanbul, Turkey.

${ }^{\mathrm{xxx}}$ Also at Texas A\&M University at Qatar, Doha, Qatar.

${ }^{\text {yyy }}$ Also at Kyungpook National University, Daegu, Korea. 